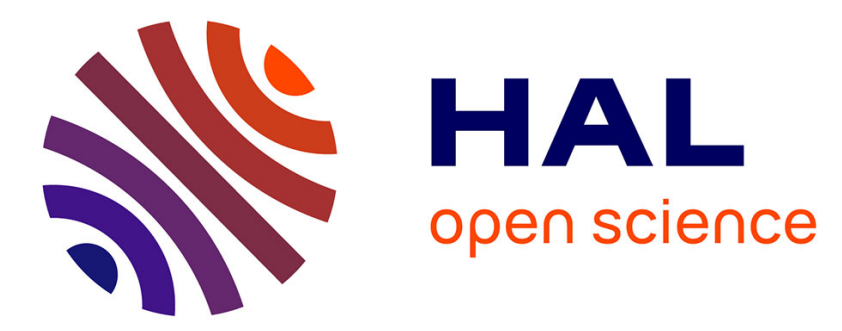

\title{
Testing slope homogeneity in large panels
}

M. Hashem Pesaran, Takashi Yamagata

\section{To cite this version:}

M. Hashem Pesaran, Takashi Yamagata. Testing slope homogeneity in large panels. Econometrics, 2007, 142 (1), pp.50. 10.1016/j.jeconom.2007.05.010 . hal-00501795

\section{HAL Id: hal-00501795 \\ https://hal.science/hal-00501795}

Submitted on 12 Jul 2010

HAL is a multi-disciplinary open access archive for the deposit and dissemination of scientific research documents, whether they are published or not. The documents may come from teaching and research institutions in France or abroad, or from public or private research centers.
L'archive ouverte pluridisciplinaire HAL, est destinée au dépôt et à la diffusion de documents scientifiques de niveau recherche, publiés ou non, émanant des établissements d'enseignement et de recherche français ou étrangers, des laboratoires publics ou privés. 


\section{Author's Accepted Manuscript}

Testing slope homogeneity in large panels

M. Hashem Pesaran, Takashi Yamagata

PII: S0304-4076(07)00122-4

DOI: $\quad$ doi:10.1016/j.jeconom.2007.05.010

Reference: $\quad$ ECONOM 2951

To appear in: $\quad$ Journal of Econometrics

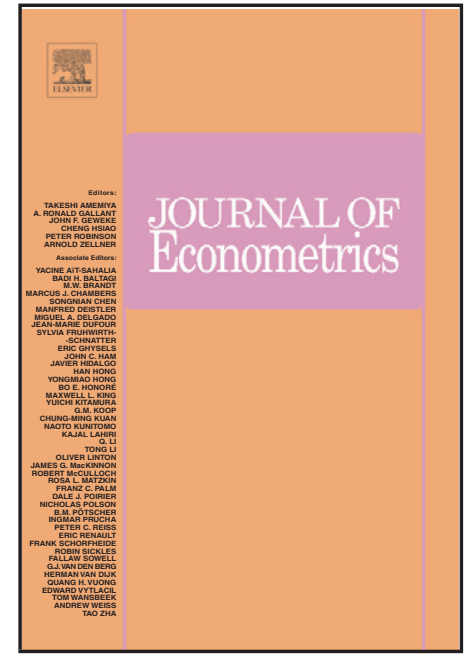

www.elsevier.com/locate/jeconom

Cite this article as: M. Hashem Pesaran and Takashi Yamagata, Testing slope homogeneity in large panels, Journal of Econometrics (2007), doi:10.1016/j.jeconom.2007.05.010

This is a PDF file of an unedited manuscript that has been accepted for publication. As a service to our customers we are providing this early version of the manuscript. The manuscript will undergo copyediting, typesetting, and review of the resulting galley proof before it is published in its final citable form. Please note that during the production process errors may be discovered which could affect the content, and all legal disclaimers that apply to the journal pertain. 


\title{
Testing Slope Homogeneity in Large Panels*
}

\author{
M. Hashem Pesaran \\ University of Cambridge and USC
}

\author{
Takashi Yamagata \\ CERF, Judge Business School, \\ University of Cambridge
}

First Version January 2005, This Version 21 March 2007

\begin{abstract}
This paper proposes a standardized version of Swamy's test of slope homogeneity for panel data models where the cross section dimension $(N)$ could be large relative to the time series dimension $(T)$. The proposed test, denoted by $\tilde{\Delta}$, exploits the cross section dispersion of individual slopes weighted by their relative precision. In the case of models with strictly exogenous regressors, but with non-normally distributed errors, the test is shown to have a standard normal distribution as $(N, T) \stackrel{j}{\rightarrow} \infty$ such that $\sqrt{N} / T^{2} \rightarrow 0$. When the errors are normally distributed, a mean-variance bias adjusted version of the test is shown to be normally distributed irrespective of the relative expansion rates of $N$ and $T$. The test is also applied to stationary dynamic models, and shown to be valid asymptotically so long as $N / T \rightarrow \kappa$, as $(N, T) \stackrel{j}{\rightarrow} \infty$, where $0 \leq \kappa<\infty$. Using Monte Carlo experiments, it is shown that the test has the correct size and satisfactory power in panels with strictly exogenous regressors for various combinations of $N$ and $T$. Similar results are also obtained for dynamic panels, but only if the autoregressive coefficient is not too close to unity and so long as $T \geq N$.
\end{abstract}

JEL Classifications: C12, C33.

Key Words: Tests of Slope Homogeneity, Dispersion Tests, Large Panels, Monte Carlo Results.

\footnotetext{
${ }^{*}$ We would like to thank Ron Smith and Aman Ullah for helpful discussions. We are particularly grateful to Cheng Hsiao and to three anonymous referees for their detailed, constructive and most helpful comments. Also our thanks go to Donggyu Sul for the Gauss codes used for the implementation of Phillips and Sul's G test. Financial support from the ESRC (Grant No. RES-000-23-0135) is gratefully acknowledged.
} 


\section{Introduction}

In many empirical studies, it is assumed that the slope coefficients of interest in panel data models are homogeneous across individual units. When the cross section dimension $(N)$ is relatively small and the time series dimension of the panel $(T)$ large, the hypothesis of slope homogeneity can be tested using the SURE (seemingly unrelated regression equation) framework of Zellner (1962). This framework is particularly attractive as it also automatically deals with the possibility of cross section error correlations and dynamics when $N$ is reasonably small (around 5-10) and $T$ sufficiently large (around 80-100). However, in many microeconometric applications $N$ is often much larger than $T$ and the SURE approach would not be applicable.

In view of this Pesaran, Smith and Im (1996) proposed the application of the Hausman (1978) testing procedure where the standard fixed effects estimator is compared to the mean group estimator. However, as will be discussed below, such a procedure is not applicable in the case of panel data models that contain only strictly exogenous regressors and/or in the case of pure autoregressive models. Recently Phillips and Sul (2003) have also proposed a 'Hausman type' test for slope homogeneity for stationary first-order autoregression $(\mathrm{AR}(1))$ panel data models in presence of cross section dependence, with $N$ fixed as $T$ goes to infinity. It will be shown below that their testing approach is not valid under cross section independence.

This paper proposes dispersion type tests based on the early work of Swamy (1970) that are applicable to panel data models where the cross section dimension could be large relative to the time series dimension. One version of the test, denoted by $\hat{\Delta}$, makes use of the Swamy statistic, $\hat{S}$, and another version, denoted by $\tilde{\Delta}$, is based on a modified version of the Swamy statistic where regression standard errors for the individual cross section units are computed using the pooled fixed effects, rather than the ordinary least squares estimator. It is shown that in the case of models with strictly exogenous regressors, but with non-normal errors, both versions of the $\Delta$ test tend to the standard normal distribution as $(N, T) \stackrel{j}{\rightarrow} \infty$, subject to certain restrictions on the relative expansion rates of $N$ and $T$. For the $\hat{\Delta}$ we require $\sqrt{N} / T \rightarrow 0$, as $(N, T) \stackrel{j}{\rightarrow} \infty$, whilst for $\tilde{\Delta}$ test the condition is less restrictive and is given by $\sqrt{N} / T^{2} \rightarrow 0$. When the errors are normally distributed mean-variance bias adjusted versions of the $\Delta$ tests, denoted by $\hat{\Delta}_{a d j}$ and $\tilde{\Delta}_{a d j}$, are proposed that are valid as $(N, T) \stackrel{j}{\rightarrow} \infty$ without any restrictions on the relative expansion rates of $N$ and $T$.

The paper also considers the problem of testing homogeneity of slopes in the case of stationary dynamic models, and shows that under the null hypothesis $\tilde{\Delta}$ tends to the standard normal distribution so long as $N / T \rightarrow \kappa$, as $(N, T) \stackrel{j}{\rightarrow} \infty$, where $0 \leq \kappa<\infty$. This condition is more restrictive than the one obtained for panels with exogenous regressors, but is the same as the condition required for the validity of fixed effects estimator of the slope in $\operatorname{AR}(1)$ models in large $N$ and $T$ panels derived independently by Hahn and Kuersteiner (2002) and Alvarez and Arellano (2003).

The small sample properties of the proposed tests are investigated along with the existing tests of slope homogeneity (namely the Hausman and Swamy's tests) by means of Monte Carlo experiments. In these experiments we focus on the $\tilde{\Delta}_{a d j}$ test, and show that for panels with different number of exogenous regressors (1 to 4 ), the test has the correct size for all combinations of $T=10,20,30,50,100,200$ and $N=20,30,50,100,200$, and is robust to non-normal errors. It also has good power properties, with the power rising with both $T$ and $N$, but more rapidly with $T$ than with $N$, as predicted by the asymptotic theory. This is in contrast to the results obtained for the Swamy's test that exhibit significant over-rejections particularly for values of $N>T$. Also as predicted by our theoretical analysis, the Hausman test has the correct size but lacks power in the case of panels with exogenous regressors and randomly distributed slopes under the alternatives. Similar results are also obtained for dynamic panels, but only if the autoregressive coefficient $(\beta)$ 
is not too close to unity and so long as $T \geq N$. In cases where $N>T$ and/or $\beta$ is close to unity a bootstrap version of the $\Delta$ test might be required. This is the subject of our on going research.

The plan of the paper is as follows. Section 2 sets up the model and reviews existing tests of slope homogeneity. Section 3 considers the asymptotic distribution of alternative dispersion type tests of slope homogeneity and establishes their asymptotic distribution in the context of panel data models where $N$ could be large relative to $T$. Section 4 considers the application of the proposed $\tilde{\Delta}$ test to stationary dynamic panel data models. Section 5 sets up the Monte Carlo design and summarizes the small sample results. Section 6 provides some concluding remarks.

Notations: $K$ stands for a finite positive constant, $\|\mathbf{A}\|=\left[\operatorname{Tr}\left(\mathbf{A} \mathbf{A}^{\prime}\right)\right]^{1 / 2}$ is the Euclidean norm of the $m \times n$ matrix $\mathbf{A}, a_{n}=O\left(b_{n}\right)$ states the deterministic sequence $\left\{a_{n}\right\}$ is at most of order $b_{n}, \mathbf{x}_{n}=O_{p}\left(\mathbf{y}_{n}\right)$ states the vector of random variables, $\mathbf{x}_{n}$, is at most of order $\mathbf{y}_{n}$ in probability, $\rightarrow_{p}$ convergence in probability, $\rightarrow_{d}$ convergence in distribution, and $\stackrel{d}{\sim}$ asymptotic equivalence of probability distributions. All asymptotics are carried under $(N, T) \stackrel{j}{\rightarrow} \infty$, which denote joint convergence of $N$ and $T \rightarrow \infty$. Restrictions (if any) on the relative expansion rates of $N$ and $T$ will be specified separately.

\section{The Model and Existing Tests of Slope Homogeneity}

Consider the panel data model with fixed effects and heterogeneous slopes

$$
y_{i t}=\alpha_{i}+\boldsymbol{\beta}_{i}^{\prime} \mathbf{x}_{i t}+\varepsilon_{i t}, i=1, \ldots, N, t=1, \ldots, T
$$

where $\alpha_{i}$ is bounded on a compact set, $\mathbf{x}_{i t}$ is a $k \times 1$ vector of strictly exogenous regressors, $\boldsymbol{\beta}_{i}$ is a $k \times 1$ vector of unknown slope coefficients, such that $\left\|\boldsymbol{\beta}_{i}\right\|<K$. Stacking the time series observations for $i$ yields

$$
\mathbf{y}_{i}=\alpha_{i} \boldsymbol{\tau}_{T}+\mathbf{X}_{i} \boldsymbol{\beta}_{i}+\boldsymbol{\varepsilon}_{i}, i=1,2, . ., N
$$

where $\mathbf{y}_{i}=\left(y_{i 1}, \ldots, y_{i T}\right)^{\prime}, \boldsymbol{\tau}_{T}$ is a $T \times 1$ vector of ones, $\mathbf{X}_{i}=\left(\mathbf{x}_{i 1}, \ldots, \mathbf{x}_{i T}\right)^{\prime}$, and $\boldsymbol{\varepsilon}_{i}=\left(\varepsilon_{i 1}, \ldots, \varepsilon_{i T}\right)^{\prime}$. The null hypothesis of interest is

$$
H_{0}: \boldsymbol{\beta}_{i}=\boldsymbol{\beta} \text { for all } i
$$

against the alternatives

$$
H_{1}: \boldsymbol{\beta}_{i} \neq \boldsymbol{\beta}_{j} \text {, for a non-zero fraction of pairwise slopes for } i \neq j \text {. }
$$

\subsection{The Standard F Test}

There are a number of procedures that can be used to test $H_{0}$, the most familiar of which is the standard $F$ statistic defined by

$$
F=\left[\frac{N(T-k-1)}{k(N-1)}\right]\left(\frac{R S S R-U S S R}{U S S R}\right),
$$

where $R S S R$ and $U S S R$ are restricted and unrestricted residual sum of squares, respectively, obtained under the null $\left(\boldsymbol{\beta}_{i}=\boldsymbol{\beta}\right)$ and the alternative hypotheses. A test based on $F$ is valid for a fixed $N$, and when the regressors are strictly exogenous and the error variances are homoskedastic, $\sigma_{i}^{2}=\sigma^{2}$. Under these assumptions and assuming $H_{0}$ holds, it is distributed as $F$ with $k(N-1)$ and $N(T-k-1)$ degrees of freedom. 


\subsection{Hausman Type Test by Pesaran, Smith and Im}

For cases where $N>T$, Pesaran, Smith and Im (1996) propose using the Hausman (1978) test where the standard fixed effects (FE) estimator of $\boldsymbol{\beta}$,

$$
\hat{\boldsymbol{\beta}}_{F E}=\left(\sum_{i=1}^{N} \mathbf{X}_{i}^{\prime} \mathbf{M}_{\tau} \mathbf{X}_{i}\right)^{-1} \sum_{i=1}^{N} \mathbf{X}_{i}^{\prime} \mathbf{M}_{\tau} \mathbf{y}_{i},
$$

is compared to the mean group (MG) estimator defined by

$$
\hat{\boldsymbol{\beta}}_{M G}=N^{-1} \sum_{i=1}^{N} \hat{\boldsymbol{\beta}}_{i},
$$

where $\mathbf{M}_{\tau}=\mathbf{I}_{T}-\boldsymbol{\tau}_{T}\left(\boldsymbol{\tau}_{T}^{\prime} \boldsymbol{\tau}_{T}\right)^{-1} \boldsymbol{\tau}_{T}^{\prime}, \mathbf{I}_{T}$ is an identity matrix of order $T$, and

$$
\hat{\boldsymbol{\beta}}_{i}=\left(\mathbf{X}_{i}^{\prime} \mathbf{M}_{\tau} \mathbf{X}_{i}\right)^{-1} \mathbf{X}_{i}^{\prime} \mathbf{M}_{\tau} \mathbf{y}_{i} .
$$

For the Hausman test to have the correct size and be consistent two conditions must be met:

(a) Under $H_{0}, \hat{\boldsymbol{\beta}}_{F E}$ and $\hat{\boldsymbol{\beta}}_{M G}$ must both be consistent, with $\hat{\boldsymbol{\beta}}_{F E}$ being asymptotically more efficient such that

$$
A V a r\left(\hat{\boldsymbol{\beta}}_{M G}-\hat{\boldsymbol{\beta}}_{F E}\right)=A \operatorname{Var}\left(\hat{\boldsymbol{\beta}}_{M G}\right)-\operatorname{AVar}\left(\hat{\boldsymbol{\beta}}_{F E}\right)>0
$$

where $A \operatorname{Var}(\cdot)$ stands for the asymptotic variance operator.

(b) Under $H_{1}, \hat{\boldsymbol{\beta}}_{M G}-\hat{\boldsymbol{\beta}}_{F E}$ should tend to a non-zero vector.

In the context of dynamic panel data models with exogenous regressors both of these conditions are met, so long as the exogenous regressors are not drawn from the same distribution. In such a case a Hausman type test based on the difference $\hat{\boldsymbol{\beta}}_{F E}-\hat{\boldsymbol{\beta}}_{M G}$ would be valid and is shown to have reasonable small sample properties. See Pesaran, Smith and Im (1996) and Hsiao and Pesaran (2007).

However, as is well known the Hausman procedure could lack power for certain parameter values, as its implicit null does not necessarily coincide with the null hypothesis of interest. Holly (1982) provides a general discussion of this point. This problem is, however, much more serious in the application of the Hausman procedure to the testing problem that concerns us here. For example, in the case of panel data models containing only strictly exogenous regressors a test of slope homogeneity based on $\hat{\boldsymbol{\beta}}_{F E}-\hat{\boldsymbol{\beta}}_{M G}$ will lack power in all directions, if under the alternative hypothesis the slopes are random draws from the same distribution. To see this suppose that under $H_{1}$ the slopes satisfy the familiar random coefficient specification

$$
\boldsymbol{\beta}_{i}=\boldsymbol{\beta}+\mathbf{v}_{i}, \mathbf{v}_{i} \sim \operatorname{IID}\left(\mathbf{0}, \boldsymbol{\Sigma}_{v}\right),
$$

where $\boldsymbol{\Sigma}_{v} \neq \mathbf{0}$ is a non-negative definite matrix, and $E\left(\mathbf{X}_{j}^{\prime} \mathbf{v}_{i}\right)=\mathbf{0}$ for all $i$ and $j$. Then

$$
\begin{aligned}
\hat{\boldsymbol{\beta}}_{F E}-\hat{\boldsymbol{\beta}}_{M G}= & \left(\sum_{i=1}^{N} \mathbf{X}_{i}^{\prime} \mathbf{M}_{\tau} \mathbf{X}_{i}\right)^{-1} \sum_{i=1}^{N}\left(\mathbf{X}_{i}^{\prime} \mathbf{M}_{\tau} \mathbf{X}_{i}\right) \mathbf{v}_{i}-N^{-1} \sum_{i=1}^{N} \mathbf{v}_{i}+ \\
& \left(\sum_{i=1}^{N} \mathbf{X}_{i}^{\prime} \mathbf{M}_{\tau} \mathbf{X}_{i}\right)^{-1} \sum_{i=1}^{N} \mathbf{X}_{i}^{\prime} \mathbf{M}_{\tau} \varepsilon_{i}-N^{-1} \sum_{i=1}^{N}\left(\mathbf{X}_{i}^{\prime} \mathbf{M}_{\tau} \mathbf{X}_{i}\right)^{-1} \mathbf{X}_{i}^{\prime} \mathbf{M}_{\tau} \varepsilon_{i},
\end{aligned}
$$


and it readily follows that under the random coefficients alternatives and strictly exogenous regressors, we have $E\left(\hat{\boldsymbol{\beta}}_{F E}-\hat{\boldsymbol{\beta}}_{M G} \mid H_{1}\right)=\mathbf{0}$. This result holds for $N$ and $T$ fixed as well as when $N$ and $T \rightarrow \infty$, and hence condition (b) of Hausman's procedure is not satisfied.

Another important case where the Hausman test does not apply arises when testing the homogeneity of slopes in pure autoregressive panel data models. To simplify the exposition consider the following stationary $\mathrm{AR}(1)$ panel data model

$$
y_{i t}=\alpha_{i}\left(1-\beta_{i}\right)+\beta_{i} y_{i, t-1}+\varepsilon_{i t} .
$$

It is now easily seen that with $N$ fixed and as $T \rightarrow \infty$, under $H_{0}$ (where $\beta_{i}=\beta$ ) we have

$$
\sqrt{N T}\left(\hat{\beta}_{F E}-\beta\right) \rightarrow_{d} N\left(0,1-\beta^{2}\right),
$$

and

$$
\sqrt{N T}\left(\hat{\beta}_{M G}-\beta\right) \rightarrow_{d} N\left(0,1-\beta^{2}\right) .
$$

Hence the variance inequality part of condition (a), namely (9), is not satisfied, and the application of the Hausman test to autoregressive panels will fail to have the correct size.

\section{$2.3 \quad G$ Test of Phillips and Sul}

Phillips and Sul (2003) propose a different type of Hausman test where instead of comparing two different pooled estimators of the regression coefficients (as discussed above), they propose basing the test of slope homogeneity on the difference between the individual estimates and a suitably defined pooled estimator. In the context of the panel regression model (2), their test statistic can be written as

$$
G=\left(\hat{\boldsymbol{\beta}}_{N}-\boldsymbol{\tau}_{N} \otimes \hat{\boldsymbol{\beta}}_{F E}\right)^{\prime} \hat{\boldsymbol{\Sigma}}_{g}^{-1}\left(\hat{\boldsymbol{\beta}}_{N}-\boldsymbol{\tau}_{N} \otimes \hat{\boldsymbol{\beta}}_{F E}\right),
$$

where $\hat{\boldsymbol{\beta}}_{N}=\left(\hat{\boldsymbol{\beta}}_{1}^{\prime}, \hat{\boldsymbol{\beta}}_{2}^{\prime}, \ldots, \hat{\boldsymbol{\beta}}_{N}^{\prime}\right)^{\prime}$ is an $N k \times 1$ stacked vector of all the $N$ individual least square estimates, $\hat{\boldsymbol{\beta}}_{F E}$ is a fixed effect estimator as before, and $\hat{\boldsymbol{\Sigma}}_{g}$ is a consistent estimator of $\boldsymbol{\Sigma}_{g}$, the asymptotic variance matrix of $\hat{\boldsymbol{\beta}}_{N}-\boldsymbol{\tau}_{N} \otimes \hat{\boldsymbol{\beta}}_{F E}$, under $H_{0} \cdot{ }^{1}$ Under standard assumptions for stationary dynamic models (see Assumption D1-D4 below), and assuming $H_{0}$ holds and $N$ is fixed, then $G \rightarrow_{d} \chi^{2}(N k)$ as $T \rightarrow \infty$, so long as the $\boldsymbol{\Sigma}_{g}$ is a non-stochastic positive definite matrix.

As compared to the Hausman test based on $\hat{\boldsymbol{\beta}}_{M G}-\hat{\boldsymbol{\beta}}_{F E}$, the $G$ test is likely to be more powerful; but its use will be limited to panel data models where $N$ is small relative to $T$. Also, the $G$ test will not be valid in the case of pure dynamic models, very much for the same kind of reasons noted above in relation to the Hausman test based on $\hat{\boldsymbol{\beta}}_{M G}-\hat{\boldsymbol{\beta}}_{F E}$. This is easily established in the case of the stationary first order autoregressive panel data model considered by Phillips and Sul (2003). Consider the AR(1) specification given by (10), and for simplicity impose homoskedastic assumption, $\sigma_{i}^{2}=\sigma^{2}$, for all $i$. It is then easily verified that under $H_{0}$

$$
\begin{aligned}
\operatorname{Avar}\left[\sqrt{T}\left(\hat{\beta}_{i}-\hat{\beta}_{F E}\right)\right] & =\operatorname{Avar}\left[\sqrt{T}\left(\hat{\beta}_{i}-\beta\right)-\sqrt{T}\left(\hat{\beta}_{F E}-\beta\right)\right] \\
& =\left(1-\beta^{2}\right)-\left(\frac{1-\beta^{2}}{N}\right), \\
\operatorname{Acov}\left[\sqrt{T}\left(\hat{\beta}_{i}-\hat{\beta}_{F E}\right), \sqrt{T}\left(\hat{\beta}_{j}-\hat{\beta}_{F E}\right)\right] & =-\left(\frac{1-\beta^{2}}{N}\right) .
\end{aligned}
$$

\footnotetext{
${ }^{1}$ Phillips and Sul consider a number of different estimators, including Andrew's (1993) median unbiased estimator and its extension to panels. But, as they note, all such estimators yield the same asymptotic covariance matrix as $T \rightarrow \infty$.
} 
Therefore

$$
\boldsymbol{\Sigma}_{g}=\left(\frac{1-\beta^{2}}{T}\right)\left(\mathbf{I}_{N}-N^{-1} \boldsymbol{\tau}_{N} \boldsymbol{\tau}_{N}^{\prime}\right)
$$

where $\operatorname{Rank}\left(\boldsymbol{\Sigma}_{g}\right)=N-1$ and $\boldsymbol{\Sigma}_{g}$ is non-invertible.

\subsection{Swamy's Test}

Swamy (1970) bases his test of slope homogeneity on the dispersion of individual slope estimates from a suitable pooled estimator. Like the $F$ test, Swamy's test is developed for panels where $N$ is small relative to $T$, but allows for cross section heteroskedasticity. Swamy's statistic applied to the slope coefficients can be written as

$$
\hat{S}=\sum_{i=1}^{N}\left(\hat{\boldsymbol{\beta}}_{i}-\hat{\boldsymbol{\beta}}_{W F E}\right)^{\prime} \frac{\mathbf{X}_{i}^{\prime} \mathbf{M}_{\tau} \mathbf{X}_{i}}{\hat{\sigma}_{i}^{2}}\left(\hat{\boldsymbol{\beta}}_{i}-\hat{\boldsymbol{\beta}}_{W F E}\right),
$$

where

$$
\hat{\sigma}_{i}^{2}=\frac{\left(\mathbf{y}_{i}-\mathbf{X}_{i} \hat{\boldsymbol{\beta}}_{i}\right)^{\prime} \mathbf{M}_{\tau}\left(\mathbf{y}_{i}-\mathbf{X}_{i} \hat{\boldsymbol{\beta}}_{i}\right)}{(T-k-1)},
$$

and $\hat{\boldsymbol{\beta}}_{W F E}$ is the weighted FE (WFE) pooled estimator of slope coefficients defined by

$$
\hat{\boldsymbol{\beta}}_{W F E}=\left(\sum_{i=1}^{N} \frac{\mathbf{X}_{i}^{\prime} \mathbf{M}_{\tau} \mathbf{X}_{i}}{\hat{\sigma}_{i}^{2}}\right)^{-1} \sum_{i=1}^{N} \frac{\mathbf{X}_{i}^{\prime} \mathbf{M}_{\tau} \mathbf{y}_{i}}{\hat{\sigma}_{i}^{2}} .
$$

In the case where $N$ is fixed and $T$ tends to infinity, under $H_{0}$ the Swamy statistic, $\hat{S}$, is asymptotically chi-square-distributed with $k(N-1)$ degrees of freedom. ${ }^{2}$

\section{Dispersion Type Tests for Large Panels}

Our survey of the literature suggests that there are no satisfactory tests of slope homogeneity in panels where $N$ is large relative to $T$. The standard $F$ test and its extension by Swamy (1970) are appropriate for panels where $N$ is small relative to $T$. Hausman type tests advanced by Pesaran, Smith and Im (1996) apply to large $N$ panels, but are not generally applicable and can suffer from low power. In this paper we propose standardized dispersion statistics that are asymptotically normally distributed as $(N, T) \stackrel{j}{\rightarrow} \infty$, with certain condition on the relative expansion rates of $N$ and $T$, if any.

In addition to Swamy's test statistic, $\hat{S}$, defined by (11), we also consider the following modified version

$$
\tilde{S}=\sum_{i=1}^{N}\left(\hat{\boldsymbol{\beta}}_{i}-\tilde{\boldsymbol{\beta}}_{W F E}\right)^{\prime} \frac{\mathbf{X}_{i}^{\prime} \mathbf{M}_{\tau} \mathbf{X}_{i}}{\tilde{\sigma}_{i}^{2}}\left(\hat{\boldsymbol{\beta}}_{i}-\tilde{\boldsymbol{\beta}}_{W F E}\right),
$$

where instead of $\hat{\sigma}_{i}^{2}$, we use $\tilde{\sigma}_{i}^{2}$ which is based on $\tilde{\boldsymbol{\beta}}_{F E}$, namely

$$
\tilde{\sigma}_{i}^{2}=\frac{\left(\mathbf{y}_{i}-\mathbf{X}_{i} \hat{\boldsymbol{\beta}}_{F E}\right)^{\prime} \mathbf{M}_{\tau}\left(\mathbf{y}_{i}-\mathbf{X}_{i} \hat{\boldsymbol{\beta}}_{F E}\right)}{T-1},
$$

\footnotetext{
${ }^{2}$ See also Hsiao (2003, p.149).
} 
and in place of $\hat{\boldsymbol{\beta}}_{W F E}$ we use $\tilde{\boldsymbol{\beta}}_{W F E}$ which is the weighted FE estimator computed using $\tilde{\sigma}_{i}^{2}$ (instead of $\hat{\sigma}_{i}^{2}$, namely

$$
\tilde{\boldsymbol{\beta}}_{W F E}=\left(\sum_{i=1}^{N} \frac{\mathbf{X}_{i}^{\prime} \mathbf{M}_{\tau} \mathbf{X}_{i}}{\tilde{\sigma}_{i}^{2}}\right)^{-1} \sum_{i=1}^{N} \frac{\mathbf{X}_{i}^{\prime} \mathbf{M}_{\tau} \mathbf{y}_{i}}{\tilde{\sigma}_{i}^{2}} .
$$

Although the difference between $\hat{S}$ and $\tilde{S}$ might appear slight at first, as we shall see below the choice of the estimator of $\sigma_{i}^{2}$ can have important implications for the properties of the two dispersion tests as $N$ and $T$ tends to infinity.

\section{1 $\Delta$ Tests}

As set out above the two versions of the Swamy's statistics, $\hat{S}$ and $\tilde{S}$, are valid for a fixed $N$ and as $T \rightarrow \infty$. In this section we consider tests based on $\hat{S}$ and $\tilde{S}$ for panels where $N$ and $T$ are both large, and establish relative expansion rates of $N$ and $T$ under which the new tests are asymptotically valid. We refer to these tests as $\Delta$ tests, and denote the standardized test statistics corresponding to $\hat{S}$ and $\tilde{S}$, by $\hat{\Delta}$, and $\tilde{\Delta}$, respectively. To this end first let

$$
\begin{gathered}
\mathbf{Q}_{i T}=T^{-1}\left(\mathbf{X}_{i}^{\prime} \mathbf{M}_{\tau} \mathbf{X}_{i}\right), \\
\mathbf{Q}_{N T}=(N T)^{-1}\left(\sum_{i=1}^{N} \mathbf{X}_{i}^{\prime} \mathbf{M}_{\tau} \mathbf{X}_{i}\right), \\
\mathbf{P}_{i}=\mathbf{M}_{\tau} \mathbf{X}_{i}\left(\mathbf{X}_{i}^{\prime} \mathbf{M}_{\tau} \mathbf{X}_{i}\right)^{-1} \mathbf{X}_{i}^{\prime} \mathbf{M}_{\tau}, \\
\mathbf{M}_{i}=\mathbf{I}_{T}-\mathbf{Z}_{i}\left(\mathbf{Z}_{i}^{\prime} \mathbf{Z}_{i}\right)^{-1} \mathbf{Z}_{i}^{\prime},
\end{gathered}
$$

where $\mathbf{Z}_{i}=\left(\boldsymbol{\tau}_{T}, \mathbf{X}_{i}\right)$, and consider the following assumptions:

\section{Assumption 1:}

(i) $\varepsilon_{i t} \mid \mathbf{X}_{i} \sim \operatorname{IID}\left(0, \sigma_{i}^{2}\right), \sigma_{\max }^{2}=\max _{1 \leq i \leq N}\left(\sigma_{i}^{2}\right)<K$, and $\sigma_{\min }^{2}=\min _{1 \leq i \leq N}\left(\sigma_{i}^{2}\right)>0$.

(ii) $\varepsilon_{i t}$ and $\varepsilon_{j s}$ are independently distributed for $i \neq j$ and/or $t \neq s$,

(iii) $E\left(\varepsilon_{i t}^{9} \mid \mathbf{X}_{i}\right)<K$.

\section{Assumption 2:}

(i) The $k \times k$ matrices $\mathbf{Q}_{i T}, i=1,2, \ldots, N$, defined by (16) are positive definite and bounded, $\max _{1 \leq i \leq N} E\left\|\mathbf{Q}_{i T}\right\|<K$, and $\mathbf{Q}_{i T}$ tends to a non-stochastic positive definite matrix, $\mathbf{Q}_{i}$, $\max _{1 \leq i \leq N} E\left\|\mathbf{Q}_{i}\right\|<K$, as $T \rightarrow \infty$.

(ii) The $k \times k$ pooled observation matrix $\mathbf{Q}_{N T}$ defined by (17) is positive definite, and $\mathbf{Q}_{N T}$ tends to a non-stochastic positive definite matrix, $\mathbf{Q}=\lim _{N \rightarrow \infty} N^{-1} \sum_{i=1}^{N} \mathbf{Q}_{i}$, as $(N, T) \stackrel{j}{\rightarrow} \infty$.

\section{Assumption 3:}

There exists a finite $T_{0}$ such that for $T>T_{0}, E\left\{\left[\boldsymbol{v}_{i}^{\prime} \mathbf{M}_{\tau} \boldsymbol{v}_{i} /(T-1)\right]^{-4-\epsilon}\right\}<K$ and $E\left\{\left[\boldsymbol{v}_{i}^{\prime} \mathbf{M}_{i} \boldsymbol{v}_{i} /(T-\right.\right.$ $\left.k-1)]^{-4-\epsilon}\right\}<K$, for each $i$ and for some small positive constant $\epsilon$, where $\boldsymbol{v}_{i}=\varepsilon_{i} / \sigma_{i}$.

\section{Assumption 4:}

Under $H_{1}$, the fraction of slopes that are not the same does not tend to zero as $N \rightarrow \infty$. 
The following theorem establishes the asymptotic expansions of the two dispersion statistics.

Theorem 1 Consider the panel data model (1), and suppose that Assumptions 1-3 hold. Then under $H_{0}$, the dispersion statistics $\hat{S}$ and $\tilde{S}$ defined by (11) and (13), respectively, can be written as

$$
\begin{aligned}
& N^{-1 / 2} \hat{S}=N^{-1 / 2} \sum_{i=1}^{N} \hat{z}_{i T}+O_{p}\left(N^{-1 / 2}\right)+O_{p}\left(T^{-1 / 2}\right), \\
& N^{-1 / 2} \tilde{S}=N^{-1 / 2} \sum_{i=1}^{N} \tilde{z}_{i T}+O_{p}\left(N^{-1 / 2}\right)+O_{p}\left(T^{-1 / 2}\right),
\end{aligned}
$$

where

$$
\hat{z}_{i T}=\frac{(T-k-1) \boldsymbol{v}_{i}^{\prime} \mathbf{P}_{i} \boldsymbol{v}_{i}}{\boldsymbol{v}_{i}^{\prime} \mathbf{M}_{i} \boldsymbol{v}_{i}}, \text { and } \tilde{z}_{i T}=\frac{(T-1) \boldsymbol{v}_{i}^{\prime} \mathbf{P}_{i} \boldsymbol{v}_{i}}{\boldsymbol{v}_{i}^{\prime} \mathbf{M}_{\tau} \boldsymbol{v}_{i}}
$$

See Appendix A.2 for a proof.

Remark 1 In the case where the errors, $\varepsilon_{i t}$, are normally distributed Assumption 3 is met for $T_{0}=k+11$. See Lemma 1. In the case of non-normal errors further restrictions might be required. However, following Pesaran (2007), it is possible to relax some of these conditions by developing "truncated" versions of $\hat{S}$ and $\tilde{S}$. For example, consider $\hat{S}=\sum_{i=1}^{N} \hat{s}_{i}$, where

$$
\hat{s}_{i}=\left(\hat{\boldsymbol{\beta}}_{i}-\hat{\boldsymbol{\beta}}_{W F E}\right)^{\prime} \frac{\mathbf{X}_{i}^{\prime} \mathbf{M}_{\tau} \mathbf{X}_{i}}{\hat{\sigma}_{i}^{2}}\left(\hat{\boldsymbol{\beta}}_{i}-\hat{\boldsymbol{\beta}}_{W F E}\right)
$$

and note that under fairly general conditions $\hat{s}_{i} \rightarrow_{d} \chi^{2}(k)$, as $T \rightarrow \infty$. Consider now the truncated version of $\hat{S}$ defined by $\hat{S}^{*}=\sum_{i=1}^{N} \hat{s}_{i}^{*}$ where

$$
\hat{s}_{i}^{*}= \begin{cases}\hat{s}_{i}, & \text { if } \hat{s}_{i}<M_{k} \\ M_{k}, & \text { if } \hat{s}_{i} \geq M_{k},\end{cases}
$$

and $M_{k}$ is a positive constant such that $\operatorname{Pr}\left[\hat{s}_{i} \geq M_{k}\right]<\epsilon$, with $\epsilon$ a sufficiently small positive number. Given that for each $i, \hat{s}_{i}$ is approximately distributed as $\chi^{2}(k)$, the value of $M_{k}$ can also be obtained approximately. For example, with $\epsilon$ set at 0.0001 we have $M_{k}=23.51$ for $k=4$. For large $N$ and $T$ a test based on $\hat{S}$ and $\hat{S}^{*}$ will be equivalent. But the truncated version is likely to be better behaved in small samples. Monte Carlo evidence supporting this conjecture is available from the authors on request.

Under Assumptions 1-3, $\hat{z}_{i T}$ and $\tilde{z}_{i T}$ are independently (but necessarily identically) distributed random variables across $i$ with finite means and variances. Also as shown in Appendix A.3 for all $i$ we have

$$
\begin{gathered}
E\left(\hat{z}_{i T}\right)=k+O\left(T^{-1}\right), \operatorname{Var}\left(\hat{z}_{i T}\right)=2 k+O\left(T^{-1}\right), \\
E\left(\tilde{z}_{i T}\right)=k+O\left(T^{-2}\right), \operatorname{Var}\left(\tilde{z}_{i T}\right)=2 k+O\left(T^{-1}\right), \\
E\left|\hat{z}_{i T}\right|^{2+\epsilon / 2}<K, \text { and } E\left|\tilde{z}_{i T}\right|^{2+\epsilon / 2}<K .
\end{gathered}
$$

Using these results in conjunction with Theorem 1, we have 
Theorem 2 Consider the panel data model (1), suppose that the $k \times 1$ regressors, $\mathbf{x}_{i t}$, are strictly exogenous and Assumptions 1-3 hold. Then under $H_{0}$

$$
\begin{gathered}
\hat{\Delta} \rightarrow{ }_{d} N(0,1) \text {, as }(N, T) \stackrel{j}{\rightarrow} \infty, \text { so long as } \sqrt{N} / T \rightarrow 0, \\
\tilde{\Delta} \rightarrow{ }_{d} N(0,1) \text {, as }(N, T) \stackrel{j}{\rightarrow} \infty, \text { so long as } \sqrt{N} / T^{2} \rightarrow 0,
\end{gathered}
$$

where the standardized dispersion statistics, $\hat{\Delta}$ and $\tilde{\Delta}$ are defined by

$$
\begin{aligned}
& \hat{\Delta}=\sqrt{N}\left(\frac{N^{-1} \hat{S}-k}{\sqrt{2 k}}\right), \\
& \tilde{\Delta}=\sqrt{N}\left(\frac{N^{-1} \tilde{S}-k}{\sqrt{2 k}}\right) .
\end{aligned}
$$

See Appendix A.4 for a proof.

This theorem also suggests that tests of slope homogeneity based on $\tilde{\Delta}$ is likely to have better size properties than the tests based on $\hat{\Delta}$. Similar results also follow under normally distributed errors. In this case, as shown in Appendix A.5 we have

$$
E\left(\hat{z}_{i T}\right)=k+O\left(T^{-1}\right), \text { and } E\left(\tilde{z}_{i T}\right)=k,
$$

and the requirement on the relative expansion rate of $N$ and $T$ for the $\tilde{\Delta}$ test gets relaxed. The results for the normally distributed case are summarized in the following Corollary.

Corollary 1 Suppose that the conditions of Theorem 2 are met, and the errors, $\varepsilon_{i t}$, are normally distributed. Then under $H_{0}$

$$
\begin{gathered}
\hat{\Delta} \rightarrow{ }_{d} N(0,1) \text {, as }(N, T) \stackrel{j}{\rightarrow} \infty, \text { so long as } \sqrt{N} / T \rightarrow 0, \\
\tilde{\Delta} \rightarrow_{d} N(0,1) \text {, as }(N, T) \stackrel{j}{\rightarrow} \infty .
\end{gathered}
$$

See Appendix A.5 for a proof.

Remark 2 The small sample properties of the dispersion tests can be improved under the normally distributed errors by considering the following mean and variance bias adjusted versions of $\hat{\Delta}$ and $\tilde{\Delta}$

$$
\hat{\Delta}_{a d j}=\sqrt{N}\left(\frac{N^{-1} \hat{S}-E\left(\hat{z}_{i T}\right)}{\sqrt{\operatorname{Var}\left(\hat{z}_{i T}\right)}}\right), \tilde{\Delta}_{a d j}=\sqrt{N}\left(\frac{N^{-1} \tilde{S}-E\left(\tilde{z}_{i T}\right)}{\sqrt{\operatorname{Var}\left(\tilde{z}_{i T}\right)}}\right),
$$

where

$$
\begin{aligned}
& E\left(\hat{z}_{i T}\right)=\frac{k(T-k-1)}{T-k-3}, \operatorname{Var}\left(\hat{z}_{i T}\right)=\frac{2 k(T-k-1)^{2}(T-3)}{(T-k-3)^{2}(T-k-5)}, \\
& E\left(\tilde{z}_{i T}\right)=k, \operatorname{Var}\left(\tilde{z}_{i T}\right)=\frac{2 k(T-k-1)}{T+1} .
\end{aligned}
$$

See Appendix A.5. 
Remark 3 The proposed testing approach can be readily extended to testing the homogeneity of a sub-set of slope coefficients. Consider the following partitioned form of (1)

$$
\underset{T \times 1}{\mathbf{y}_{i}}=\alpha_{i} \boldsymbol{\tau}_{T}+\underset{T \times k_{1}}{\mathbf{X}_{i 1}} \boldsymbol{\beta}_{i 1}+\underset{T \times k_{2}}{\mathbf{X}_{i 2}} \boldsymbol{\beta}_{i 2}+\boldsymbol{\varepsilon}_{i}, i=1,2, . ., N,
$$

or

$$
\underset{T \times 1}{\mathbf{y}_{i}}=\underset{T \times\left(k_{1}+1\right)}{\mathbf{Z}_{i 1}} \boldsymbol{\theta}_{i}+\underset{T \times k_{2}}{\mathbf{X}_{i 2}} \boldsymbol{\beta}_{i 2}+\boldsymbol{\varepsilon}_{i}
$$

where $\mathbf{Z}_{i 1}=\left(\boldsymbol{\tau}_{T}, \mathbf{X}_{i 1}\right)$ and $\boldsymbol{\theta}_{i}=\left(\alpha_{i}, \boldsymbol{\beta}_{i 1}^{\prime}\right)^{\prime}$. Suppose the slope homogeneity hypothesis of interest is given by

$$
H_{0}: \boldsymbol{\beta}_{i 2}=\boldsymbol{\beta}_{2} \text {, for } i=1,2, \ldots, N \text {. }
$$

Our version of the dispersion test statistic in this case is given by

$$
\tilde{S}_{2}=\sum_{i=1}^{N}\left(\hat{\boldsymbol{\beta}}_{i 2}-\tilde{\boldsymbol{\beta}}_{2, W F E}\right)^{\prime} \frac{\mathbf{X}_{i 2}^{\prime} \mathbf{M}_{i 1} \mathbf{X}_{i 2}}{\tilde{\sigma}_{i}^{2}}\left(\hat{\boldsymbol{\beta}}_{i 2}-\tilde{\boldsymbol{\beta}}_{2, W F E}\right),
$$

where

$$
\begin{gathered}
\hat{\boldsymbol{\beta}}_{i 2}=\left(\mathbf{X}_{i 2}^{\prime} \mathbf{M}_{i 1} \mathbf{X}_{i 2}\right)^{-1} \mathbf{X}_{i 2}^{\prime} \mathbf{M}_{i 1} \mathbf{y}_{i} \\
\tilde{\boldsymbol{\beta}}_{2, W F E}=\left(\sum_{i=1}^{N} \frac{\mathbf{X}_{i 2}^{\prime} \mathbf{M}_{i 1} \mathbf{X}_{i 2}}{\tilde{\sigma}_{i}^{2}}\right)^{-1} \sum_{i=1}^{N} \frac{\mathbf{X}_{i 2}^{\prime} \mathbf{M}_{i 1} \mathbf{y}_{i}}{\tilde{\sigma}_{i}^{2}} \\
\mathbf{M}_{i 1}=\mathbf{I}_{T}-\mathbf{Z}_{i 1}\left(\mathbf{Z}_{i 1}^{\prime} \mathbf{Z}_{i 1}\right)^{-1} \mathbf{Z}_{i 1}^{\prime} \\
\tilde{\sigma}_{i}^{2}=\frac{\left(\mathbf{y}_{i}-\mathbf{X}_{i 2} \hat{\boldsymbol{\beta}}_{2, F E}\right)^{\prime} \mathbf{M}_{i 1}\left(\mathbf{y}_{i}-\mathbf{X}_{i 2} \hat{\boldsymbol{\beta}}_{2, F E}\right)}{T-k_{1}-1}
\end{gathered}
$$

and

$$
\hat{\boldsymbol{\beta}}_{2, F E}=\left(\sum_{i=1}^{N} \mathbf{X}_{i 2}^{\prime} \mathbf{M}_{i 1} \mathbf{X}_{i 2}\right)^{-1} \sum_{i=1}^{N} \mathbf{X}_{i 2}^{\prime} \mathbf{M}_{i 1} \mathbf{y}_{i} .
$$

Using a similar line of reasoning as above, it is now easily seen that under $H_{0}$ defined by (32), and for $(N, T) \stackrel{j}{\rightarrow} \infty$, such that $\sqrt{N} / T^{2} \rightarrow 0$, then

$$
\tilde{\Delta}_{2}=\sqrt{N}\left(\frac{N^{-1} \tilde{S}_{2}-k_{2}}{\sqrt{2 k_{2}}}\right) \rightarrow_{d} N(0,1) .
$$

In the case of normally distributed errors the following mean-variance bias adjusted statistics apply

$$
\hat{\Delta}_{2, a d j}=\sqrt{N}\left(\frac{N^{-1} \hat{S}_{2}-E\left(\hat{z}_{i T}\right)}{\sqrt{\operatorname{Var}\left(\hat{z}_{i T}\right)}}\right), \tilde{\Delta}_{a d j}=\sqrt{N}\left(\frac{N^{-1} \tilde{S}_{2}-E\left(\tilde{z}_{i T}\right)}{\sqrt{\operatorname{Var}\left(\tilde{z}_{i T}\right)}}\right),
$$

where

$$
\begin{aligned}
& E\left(\hat{z}_{i T}\right)=\frac{k_{2}(T-k-1)}{T-k-3}, \operatorname{Var}\left(\hat{z}_{i T}\right)=\frac{2 k_{2}(T-k-1)^{2}\left(T-k_{1}-3\right)}{(T-k-3)^{2}(T-k-5)}, \\
& E\left(\tilde{z}_{i T}\right)=k_{2}, \operatorname{Var}\left(\tilde{z}_{i T}\right)=\frac{2 k_{2}(T-k-1)}{T-k_{1}+1}
\end{aligned}
$$


Remark 4 The proposed slope homogeneity tests can also be extended to unbalanced panels. Denoting the number of time series observations on the $i^{\text {th }}$ cross section by $T_{i}$, our version of the standardized dispersion statistic is given by

$$
\begin{gathered}
\tilde{\Delta}=\frac{1}{\sqrt{N}} \sum_{i=1}^{N}\left(\frac{\tilde{d}_{i}-k}{\sqrt{2 k}}\right), \\
\tilde{d}_{i}=\left(\hat{\boldsymbol{\beta}}_{i}-\tilde{\boldsymbol{\beta}}_{W F E}\right)^{\prime} \frac{\mathbf{X}_{i}^{\prime} \mathbf{M}_{\tau_{i}} \mathbf{X}_{i}}{\tilde{\sigma}_{i}^{2}}\left(\hat{\boldsymbol{\beta}}_{i}-\tilde{\boldsymbol{\beta}}_{W F E}\right), \\
\mathbf{X}_{i}=\left(\mathbf{x}_{i 1}, \ldots, \mathbf{x}_{i T_{i}}\right)^{\prime}, \mathbf{M}_{\tau_{i}}=\mathbf{I}_{T_{i}}-\boldsymbol{\tau}_{T_{i}}\left(\boldsymbol{\tau}_{T_{i}}^{\prime} \boldsymbol{\tau}_{T_{i}}\right)^{-1} \boldsymbol{\tau}_{T_{i}}^{\prime} \text { with } \boldsymbol{\tau}_{T_{i}} \text { being a } T_{i} \times 1 \text { vector of unity, } \\
\hat{\boldsymbol{\beta}}_{i}=\left(\mathbf{X}_{i}^{\prime} \mathbf{M}_{\tau_{i}} \mathbf{X}_{i}\right)^{-1} \mathbf{X}_{i}^{\prime} \mathbf{M}_{\tau_{i}} \mathbf{y}_{i}, \\
\mathbf{y}_{i}=\left(y_{i 1}, \ldots, y_{i T_{i}}\right)^{\prime}, \quad\left(\sum_{i=1}^{N} \frac{\mathbf{X}_{i}^{\prime} \mathbf{M}_{\tau_{i}} \mathbf{X}_{i}}{\tilde{\sigma}_{i}^{2}}\right)^{-1} \sum_{i=1}^{N} \frac{\mathbf{X}_{i}^{\prime} \mathbf{M}_{\tau_{i}} \mathbf{y}_{i}}{\tilde{\sigma}_{i}^{2}}, \\
\tilde{\sigma}_{i}^{2}=\frac{\left(\mathbf{y}_{i}-\mathbf{X}_{i} \hat{\boldsymbol{\beta}}_{F E}\right)^{\prime} \mathbf{M}_{\tau_{i}}\left(\mathbf{y}_{i}-\mathbf{X}_{i} \hat{\boldsymbol{\beta}}_{F E}\right)}{T_{i}-1},
\end{gathered}
$$

and

$$
\hat{\boldsymbol{\beta}}_{F E}=\left(\sum_{i=1}^{N} \mathbf{X}_{i}^{\prime} \mathbf{M}_{\tau_{i}} \mathbf{X}_{i}\right)^{-1} \sum_{i=1}^{N} \mathbf{X}_{i}^{\prime} \mathbf{M}_{\tau_{i}} \mathbf{y}_{i} .
$$

Our proofs go through by replacing $T$ with $\min _{1 \leq i \leq N} T_{i}$. An extension to testing the homogeneity of a sub-set of slope coefficients in the case of the unbalanced panels is straightforward and is easily derived using the result in Remark 3.

\subsection{Asymptotic Local Power of $\hat{\Delta}$ Test}

The two versions of the dispersion test have the same asymptotic power properties and for simplicity we shall focus on the $\hat{\Delta}$ test. To bound the asymptotic power we adopt the following local alternatives

$$
H_{1, N T}: \boldsymbol{\beta}_{i}=\boldsymbol{\beta}+\frac{\boldsymbol{\delta}_{i}}{N^{1 / 4} T^{1 / 2}}, i=1,2, \ldots, N,
$$

where $\boldsymbol{\delta}_{i}, i=1,2, \ldots, N$ are $k \times 1$ vectors of fixed constants, $\left\|\boldsymbol{\delta}_{i}\right\|<K$. Under Assumptions 1-3, and assuming that $H_{1, N T}$ holds we have ${ }^{3}$

$$
\hat{\Delta}=\frac{1}{\sqrt{N}} \sum_{i=1}^{N}\left(\frac{\hat{z}_{i T}-k}{\sqrt{2 k}}\right)+\frac{\psi_{N T}}{\sqrt{2 k}}+O_{p}\left(N^{-1 / 4}\right)+O_{p}\left(T^{-1 / 2}\right)
$$

where

$$
\psi_{N T}=\frac{1}{N} \sum_{i=1}^{N} \hat{\sigma}_{i}^{-2} \boldsymbol{\delta}_{i}^{\prime} \mathbf{Q}_{i T} \boldsymbol{\delta}_{i}-\left(\frac{1}{N} \sum_{i=1}^{N} \hat{\sigma}_{i}^{-2} \boldsymbol{\delta}_{i}^{\prime} \mathbf{Q}_{i T}\right)\left(\frac{1}{N} \sum_{i=1}^{N} \hat{\sigma}_{i}^{-2} \mathbf{Q}_{i T}\right)^{-1}\left(\frac{1}{N} \sum_{i=1}^{N} \hat{\sigma}_{i}^{-2} \mathbf{Q}_{i T} \boldsymbol{\delta}_{i}\right) .
$$

\footnotetext{
${ }^{3}$ For a proof see Appendix A.6.
} 
Hence, it readily follows that under $H_{1, N T}$

$$
\hat{\Delta} \rightarrow_{d} N\left(\frac{\psi}{\sqrt{2 k}}, 1\right), \text { as }(N, T) \stackrel{j}{\rightarrow} \infty
$$

where

$$
\psi=\lim _{N \rightarrow \infty}\left\{\frac{1}{N} \sum_{i=1}^{N} \sigma_{i}^{-2} \boldsymbol{\delta}_{i}^{\prime} \mathbf{Q}_{i} \boldsymbol{\delta}_{i}-\left(\frac{1}{N} \sum_{i=1}^{N} \sigma_{i}^{-2} \boldsymbol{\delta}_{i}^{\prime} \mathbf{Q}_{i}\right)\left(\frac{1}{N} \sum_{i=1}^{N} \sigma_{i}^{-2} \mathbf{Q}_{i}\right)^{-1}\left(\frac{1}{N} \sum_{i=1}^{N} \sigma_{i}^{-2} \mathbf{Q}_{i} \boldsymbol{\delta}_{i}\right)\right\} .
$$

Recall that $\mathbf{Q}_{i}=p \lim _{T \rightarrow \infty}\left(T^{-1} \mathbf{X}_{i}^{\prime} \mathbf{M}_{\tau} \mathbf{X}_{i}\right)$. The $\hat{\Delta}$ test has power against local alternatives if $\psi>0$. Since $\mathbf{Q}_{i}$ is a symmetric positive definite matrix, using the the Cholesky decomposition, $\mathbf{Q}_{i}=\mathbf{L}_{i}^{\prime} \mathbf{L}_{i}$, and setting $\tilde{\boldsymbol{\delta}}_{i}=\mathbf{L}_{i} \boldsymbol{\delta}_{i} / \sigma_{i}$, and $\mathbf{W}_{i}=\sigma_{i}^{-1} \mathbf{L}_{i}$ we have

$$
\begin{aligned}
\psi & =\lim _{N \rightarrow \infty}\left\{\frac{1}{N} \sum_{i=1}^{N} \tilde{\boldsymbol{\delta}}_{i}^{\prime} \tilde{\boldsymbol{\delta}}_{i}-\left(\frac{1}{N} \sum_{i=1}^{N} \tilde{\boldsymbol{\delta}}_{i}^{\prime} \mathbf{W}_{i}\right)\left(\frac{1}{N} \sum_{i=1}^{N} \mathbf{W}_{i}^{\prime} \mathbf{W}_{i}\right)^{-1}\left(\frac{1}{N} \sum_{i=1}^{N} \mathbf{W}_{i}^{\prime} \tilde{\boldsymbol{\delta}}_{i}\right)\right\} \\
& =\lim _{N \rightarrow \infty} \frac{1}{N}\left\{\sum_{i=1}^{N} \tilde{\boldsymbol{\delta}}_{i}^{\prime} \tilde{\boldsymbol{\delta}}_{i}-\left(\sum_{i=1}^{N} \tilde{\boldsymbol{\delta}}_{i}^{\prime} \mathbf{W}_{i}\right)\left(\sum_{i=1}^{N} \mathbf{W}_{i}^{\prime} \mathbf{W}_{i}\right)^{-1}\left(\sum_{i=1}^{N} \mathbf{W}_{i}^{\prime} \tilde{\boldsymbol{\delta}}_{i}\right)\right\} .
\end{aligned}
$$

Let $\tilde{\boldsymbol{\delta}}=\left(\tilde{\boldsymbol{\delta}}_{1}^{\prime}, \tilde{\boldsymbol{\delta}}_{2}^{\prime}, \ldots, \tilde{\boldsymbol{\delta}}_{N}^{\prime}\right)^{\prime}$, and $\mathbf{W}=\left(\mathbf{W}_{1}^{\prime}, \mathbf{W}_{2}^{\prime}, \ldots, \mathbf{W}_{N}^{\prime}\right)^{\prime}$, and write $\psi$ as

$$
\psi=\lim _{N \rightarrow \infty}\left(\frac{\tilde{\boldsymbol{\delta}}^{\prime} \mathbf{M}_{w} \tilde{\boldsymbol{\delta}}}{N}\right),
$$

where $\mathbf{M}_{w}=\mathbf{I}_{T}-\mathbf{W}\left(\mathbf{W}^{\prime} \mathbf{W}\right)^{-1} \mathbf{W}$. Hence, $\psi \geq 0$, and in general the $\hat{\Delta}$ test is asymptotically powerful if $\delta_{i} \neq 0$ for a non-zero fraction of the cross section units in the limit, as specified under Assumption 4. Such an alternative, for example, allows a sub-set of the slope coefficients and/or a sub-set of cross section units to be homogeneous.

The above result also suggests that the power of $\hat{\Delta}$ (or $\tilde{\Delta}$ ) test is likely to increase faster with $T$ than with $N$.

\section{Testing Slope Homogeneity in Autoregressive Panels}

To simplify the exposition we focus on the AR(1) model

$$
\mathbf{y}_{i}=\alpha_{i}\left(1-\beta_{i}\right) \boldsymbol{\tau}_{T}+\beta_{i} \mathbf{y}_{i,-1}+\varepsilon_{i}, \text { for } i=1,2, \ldots, N,
$$

where $\mathbf{y}_{i,-1}=\left(y_{i 0}, y_{i 1}, \ldots, y_{i T-1}\right)^{\prime}$. In this dynamic case we shall focus on the modified version of the Swamy test. The test statistic in this case is given by

$$
\tilde{S}=\sum_{i=1}^{N} \tilde{\sigma}_{i}^{-2}\left(\hat{\beta}_{i}-\tilde{\beta}_{W F E}\right)^{2}\left(\mathbf{y}_{i,-1}^{\prime} \mathbf{M}_{\tau} \mathbf{y}_{i,-1}\right)
$$

where

$$
\tilde{\beta}_{W F E}=\left(\sum_{i=1}^{N} \frac{\mathbf{y}_{i,-1}^{\prime} \mathbf{M}_{\tau} \mathbf{y}_{i,-1}}{\tilde{\sigma}_{i}^{2}}\right)^{-1} \sum_{i=1}^{N} \frac{\mathbf{y}_{i,-1}^{\prime} \mathbf{M}_{\tau} \mathbf{y}_{i}}{\tilde{\sigma}_{i}^{2}}
$$




$$
\tilde{\sigma}_{i}^{2}=\frac{\left(\mathbf{y}_{i}-\tilde{\beta}_{F E} \mathbf{y}_{i,-1}\right)^{\prime} \mathbf{M}_{\tau}\left(\mathbf{y}_{i}-\tilde{\beta}_{F E} \mathbf{y}_{i,-1}\right)}{T-1} .
$$

For future use also let

$$
\begin{gathered}
Q_{i T}=\frac{\mathbf{y}_{i,-1}^{\prime} \mathbf{M}_{\tau} \mathbf{y}_{i,-1}}{T}, \\
Q_{N T}=\frac{\sum_{i=1}^{N} \mathbf{y}_{i,-1}^{\prime} \mathbf{M}_{\tau} \mathbf{y}_{i,-1}}{N T},
\end{gathered}
$$

and consider the following assumptions:

\section{Assumption D1:}

(i) $\left\{\varepsilon_{i t}\right\}, t=1,2, \ldots, T, i=1,2, \ldots N$, are independently distributed across time and cross section units, and independently of the initial values, $y_{i 0}$, with finite moments up to order four, $E\left(\varepsilon_{i t}\right)=0, \operatorname{Var}\left(\varepsilon_{i t}\right)=\sigma_{i}^{2}, \sigma_{\max }^{2}=\max _{1 \leq i \leq N}\left(\sigma_{i}^{2}\right)<K$, and $\sigma_{\min }^{2}=\min _{1 \leq i \leq N}\left(\sigma_{i}^{2}\right)>0$.

\section{Assumption D2:}

$\left|\beta_{i}\right|<1$ for all $i, \alpha_{i}$ is bounded on a compact set, and $\lim N / T=\kappa$, as $(N, T) \stackrel{j}{\rightarrow} \infty$, where $0 \leq \kappa<\infty$.

\section{Assumption D3:}

The initial observations satisfy

$$
y_{i 0}=\alpha_{i}+u_{i 0}
$$

where $u_{i 0}, i=1,2, \ldots, N$ are distributed independently across $i$, and independently of $\varepsilon_{i}$, with $E\left(u_{i 0}\right)=0, \operatorname{Var}\left(u_{i 0}\right)=\delta_{i}^{2}, 0<\delta_{i}^{2}<K$ for all $i$, and with finite moments up to order four. ${ }^{4}$

Theorem 3 Consider the panel data model (40), and suppose that Assumptions D1-D3 hold. Then under $H_{0}$, the dispersion statistic, $\tilde{S}$, defined by (41), can be written as

$$
N^{-1 / 2} \tilde{S}=N^{-1 / 2} \sum_{i=1}^{N} \tilde{w}_{i}+O_{p}\left(N^{-1 / 2}\right)+O_{p}\left(T^{-1 / 2}\right),
$$

where

$$
\tilde{w}_{i}=\frac{(T-1)\left(T^{-1 / 2} \varepsilon_{i}^{\prime} \mathbf{M}_{\tau} \mathbf{y}_{i,-1}\right)^{2}}{\left(\varepsilon_{i}^{\prime} \mathbf{M}_{\tau} \varepsilon_{i}\right)\left(T^{-1} \mathbf{y}_{i,-1}^{\prime} \mathbf{M}_{\tau} \mathbf{y}_{i,-1}\right)}
$$

See Appendix A.7 for a proof.

It is interesting to note that the orders of the asymptotic expansion of $\tilde{S}$ for the dynamic panel considered here are the same as those of panels with exogenous regressors (see (21)). However, in the dynamic case the additional condition $N / T \rightarrow \kappa, 0 \leq \kappa<\infty$ is required. This condition ensures that the asymptotic bias of the FE estimator of $\beta$ in the dynamic case, discussed in Hahn and Kuersteiner (2002) and Alvarez and Arellano (2003), remains bounded as $(N, T) \stackrel{j}{\rightarrow} \infty$. Another important difference between the two cases is the differences that exist between the asymptotic expansions of $E\left(\tilde{z}_{i T}\right)$ and $\operatorname{Var}\left(\tilde{z}_{i T}\right)$ in the static case (given by (24)), and the corresponding expressions for $E\left(\tilde{w}_{i}\right)$ and $\operatorname{Var}\left(\tilde{w}_{i}\right)$ in the dynamic case.

\footnotetext{
${ }^{4}$ The choice of $\delta_{i}$ depends on the initialization of the process and will be given by $\delta_{i}=\sigma_{i}\left(1-\beta_{i}^{2}\right)^{-1 / 2}$ if the process has started at $t=-M$, with $M \rightarrow \infty$.
} 
To derive the order of expansions of $E\left(\tilde{w}_{i}\right)$ and $\operatorname{Var}\left(\tilde{w}_{i}\right)$ we first note that $\tilde{w}_{i}$ defined by $(47)$, can be written as

$$
\tilde{w}_{i}=\frac{\left(\boldsymbol{\nu}_{i}^{\prime} \mathbf{A}_{i} \boldsymbol{\nu}_{i}\right)^{2}}{\left(\boldsymbol{\nu}_{i}^{\prime} \mathbf{B} \boldsymbol{\nu}_{i}\right)\left(\boldsymbol{\nu}_{i}^{\prime} \mathbf{C}_{i} \boldsymbol{\nu}_{i}\right)}
$$

where $\boldsymbol{\nu}_{i}=\left(u_{i 0} / \delta_{i}, \varepsilon_{i 1} / \sigma_{i}, \ldots, \varepsilon_{i T} / \sigma_{i}\right)^{\prime} \sim \operatorname{IID}\left(\mathbf{0}, \mathbf{I}_{T+1}\right)$, and $\mathbf{A}_{i}, \mathbf{B}$, and $\mathbf{C}_{i}$ are $(T+1) \times(T+1)$ non-stochastic matrices defined in Appendix A.8 by (A.37), (A.38) and (A.39), respectively. It is clear that unlike $\tilde{z}_{i T}$ which is the ratio of quadratic forms, $\tilde{w}_{i}$ is in fact the ratio of the products of quadratic forms, and to our knowledge there exists no result in the literature on the moments of such ratios for the case of non-normal (or for that matter normal) errors.

To develop suitable expansions of $E\left(\tilde{w}_{i}\right)$ and $\operatorname{Var}\left(\tilde{w}_{i}\right)$ we introduce the following additional assumption

\section{Assumption D4:}

For each $i, \boldsymbol{\nu}_{i}=\left(u_{i 0} / \delta_{i}, \varepsilon_{i 1} / \sigma_{i}, \ldots, \varepsilon_{i T} / \sigma_{i}\right)^{\prime} \sim \operatorname{IIDN}\left(\mathbf{0}, \mathbf{I}_{T+1}\right)$, and $T>17$.

With this additional assumption the following theorem can now be established.

Theorem 4 Consider the panel data model (40), suppose that Assumptions D1-D4 hold. Then under $H_{0}, \tilde{S}$, defined by (41) satisfies

$$
\frac{1}{N} \tilde{S}=\frac{1}{N} \sum_{i=1}^{N} w_{i}^{*}+O_{p}\left(T^{-1}\right)+O_{p}\left(N^{-1}\right)+O_{p}\left(N^{-1 / 2} T^{-1 / 2}\right)
$$

where

$$
w_{i}^{*}=\frac{\left(\boldsymbol{\nu}_{i}^{\prime} \mathbf{A}_{i} \boldsymbol{\nu}_{i}\right)^{2}}{E\left[\left(\boldsymbol{\nu}_{i}^{\prime} \mathbf{B} \boldsymbol{\nu}_{i}\right)\left(\boldsymbol{\nu}_{i}^{\prime} \mathbf{C}_{i} \boldsymbol{\nu}_{i}\right)\right]}\left[1-\frac{\left(\boldsymbol{\nu}_{i}^{\prime} \mathbf{B} \boldsymbol{\nu}_{i}\right)\left(\boldsymbol{\nu}_{i}^{\prime} \mathbf{C}_{i} \boldsymbol{\nu}_{i}\right)-E\left[\left(\boldsymbol{\nu}_{i}^{\prime} \mathbf{B} \boldsymbol{\nu}_{i}\right)\left(\boldsymbol{\nu}_{i}^{\prime} \mathbf{C}_{i} \boldsymbol{\nu}_{i}\right)\right]}{E\left[\left(\boldsymbol{\nu}_{i}^{\prime} \mathbf{B} \boldsymbol{\nu}_{i}\right)\left(\boldsymbol{\nu}_{i}^{\prime} \mathbf{C}_{i} \boldsymbol{\nu}_{i}\right)\right]}\right] .
$$

Furthermore,

$$
E\left(w_{i}^{*}\right)=1+O\left(T^{-1}\right), \operatorname{Var}\left(w_{i}^{*}\right)=2+O\left(T^{-1 / 2}\right),
$$

and

$$
\tilde{\Delta} \rightarrow_{d} N(0,1), \text { as }(N, T) \stackrel{j}{\rightarrow} \infty \text {, such that } N / T \rightarrow \kappa, 0 \leq \kappa<\infty
$$

where

$$
\tilde{\Delta}=\sqrt{N}\left(\frac{N^{-1} \tilde{S}-1}{\sqrt{2}}\right)
$$

See Appendix A.8 for a proof.

One would expect that a similar result holds for higher order autoregressive models. The relevant standardized statistic for a $p^{t h}$ order process would be given by $\sqrt{\frac{N}{2 p}}\left(N^{-1} \tilde{S}-p\right)$. This is supported by Monte Carlo evidence for $\operatorname{AR}(2)$ panel specification in the following section, where we shall also examine the robustness of the test to non-normal errors.

\section{$5 \quad$ Finite Sample Properties by Monte Carlo Experiments}

In this section we investigate the finite sample properties of two main tests of slope homogeneity advanced in the literature and compare their performance to a bias adjusted version of the dispersion test proposed in this paper. The tests already available in the literature are Swamy's test based on 
$\hat{S}$ statistic defined by (11), and Hausman's test of slope homogeneity proposed in Pesaran, Smith and $\operatorname{Im}(1996)$ which in the case of application is defined by ${ }^{5}$

$$
H=\left(\hat{\boldsymbol{\beta}}_{M G}-\tilde{\boldsymbol{\beta}}_{W F E}\right)^{\prime} \hat{\mathbf{V}}_{H}^{-1}\left(\hat{\boldsymbol{\beta}}_{M G}-\tilde{\boldsymbol{\beta}}_{W F E}\right),
$$

where $\hat{\boldsymbol{\beta}}_{M G}$ and $\tilde{\boldsymbol{\beta}}_{W F E}$ are given by (7) and (15), respectively, and

$$
\hat{\mathbf{V}}_{H}=\frac{1}{N^{2}} \sum_{i=1}^{N} \hat{\sigma}_{i}^{2}\left(\mathbf{X}_{i}^{\prime} \mathbf{M}_{\tau} \mathbf{X}_{i}\right)^{-1}-\left(\sum_{i=1}^{N} \frac{\mathbf{X}_{i}^{\prime} \mathbf{M}_{\tau} \mathbf{X}_{i}}{\tilde{\sigma}_{i}^{2}}\right)^{-1}
$$

with $\hat{\sigma}_{i}^{2}$ and $\tilde{\sigma}_{i}^{2}$ being defined by (12) and (14), respectively. Under the null hypothesis of slope homogeneity, $H$ tends to a $\chi^{2}(k)$ as $T, N \rightarrow \infty$, and $\hat{S}$ tends to $\chi^{2}(k(N-1))$ for a fixed $N$ and as $T \rightarrow \infty$. We also considered the $G$ test of Phillips and Sul (2003), but the $G$ statistic could not be computed due to the singularity problem discussed in Section $2.3 .^{6}$

As far as the standardized dispersion tests proposed in this paper are concerned we considered all the four versions, namely $\hat{\Delta}$ and $\tilde{\Delta}$ defined in (26) and (27), respectively, and their mean and variance adjusted versions $\hat{\Delta}_{a d j}$ and $\tilde{\Delta}_{a d j}$, defined by (29). As to be expected the adjusted versions performed much better under normal errors, and interestingly enough turned out to be reasonably robust to non-normal errors, as well. So to save space we shall only report the results for $\tilde{\Delta}_{a d j}$ computed as

$$
\tilde{\Delta}_{a d j}=\sqrt{\frac{N(T+1)}{T-k-1}}\left(\frac{N^{-1} \tilde{S}-k}{\sqrt{2 k}}\right)
$$

where $\tilde{S}$ is defined by (13). The full set of results for all the four versions of the $\Delta$ test are available on request.

We report empirical size and power at the nominal 5 per cent level, for various pairs of $N$ and $T$, including cases where $N$ is much larger than $T$, often encountered with micro data sets, as well as when $T>N$ which is more prevalent in the case of macro data sets. We consider panels with strictly exogenous regressors, as well as simple dynamic panels. Under the former the data generating process (DGP) is defined by

$$
y_{i t}=\alpha_{i}+\sum_{\ell=1}^{k} x_{i \ell t} \beta_{i \ell}+\varepsilon_{i t}
$$

for $i=1,2, \ldots, N ; t=1,2, \ldots, T$, where $\alpha_{i} \sim N(1,1)$. The regressors, $x_{i \ell t}, \ell=1,2, \ldots, k$, are generated as

$$
x_{i \ell t}=\alpha_{i}\left(1-\rho_{i \ell}\right)+\rho_{i \ell} x_{i \ell, t-1}+\left(1-\rho_{i \ell}^{2}\right)^{1 / 2} v_{i \ell t},
$$

for $t=-48, \ldots, 0, \ldots, T ; i=1,2, \ldots, N$, where $\rho_{i \ell} \sim \operatorname{IIDU}[0.05,0.95], v_{i \ell t} \sim \operatorname{IIDN}\left(0, \sigma_{i \ell x}^{2}\right)$ with $\sigma_{i \ell x}^{2}$ $\sim I I D \chi^{2}(1) . \rho_{i \ell}$ and $\sigma_{i \ell x}^{2}$ are fixed across replications with $x_{i \ell,-49}=0$. The first 49 observations are discarded to reduce the effect of initial value on the generated values of $x_{i \ell t}, t=1,2, \ldots, T$. $\varepsilon_{i t} \sim \operatorname{IID}\left(0, \sigma_{i}^{2}\right)$ is drawn from (i) standard normal distribution, or (ii) $\left(\chi^{2}(2)-2\right) / 2$ with $\sigma_{i}^{2} \sim$ $I I D\left(k \chi^{2}(2) / 2\right), k=1,2,3,4$, so that the population $R^{2}$ of individual equations in the panel are invariant to the number of included regressors. Under the null hypothesis, $\beta_{i \ell}=1$ for all $i$ and $\ell$, and under the alternative hypothesis $\beta_{i \ell}=\beta_{i 1}$ for $\ell=2,3,4$, where $\beta_{i 1}=1$ for $i=1, \ldots,[N / 2]$ and

\footnotetext{
${ }^{5}$ We also tried a number of other variants of the Hausman test. But they all performed very similarly.

${ }^{6}$ In e-mail correspondences Dr. Sul has confirmed to us that there is an error in equation (27) in Phillips and Sul (2003) that defines the $G$ statistic.
} 
$\beta_{i 1} \sim N(1,0.04)$ for $i=[N / 2]+1, \ldots, N$, with $[N / 2]$ being the nearest integer value of $N / 2 . \alpha_{i}$ and $\sigma_{i}^{2}$ are fixed across replications. For $k=1$, all combinations of $T=10,20,30,50,100,200$ and $N=20,30,50,100,200$ are used as sample sizes. For $k=2,3,4$, to save space only the combinations of $T=20,30,50$ and $N=20,30,50,100,200$ are included.

In the dynamic case we consider pure autoregressions, $\operatorname{AR}(1)$ and $\operatorname{AR}(2)$, and a simple autoregressive distributed lag (ARDL) model. The DGP for the AR specifications are given by

$$
\begin{gathered}
y_{i t}=\left(1-\beta_{i}\right) \alpha_{i}+\beta_{i} y_{i t-1}+\varepsilon_{i t}, \\
y_{i t}=\left(1-\beta_{i 1}-\beta_{2}\right) \alpha_{i}+\beta_{i 1} y_{i t-1}+\beta_{2} y_{i t-2}+\varepsilon_{i t},
\end{gathered}
$$

for $t=-M, \ldots, 0, \ldots, T ; i=1,2, \ldots, N$, where $\alpha_{i} \sim N(1,1)$ and $\varepsilon_{i t}$ generated as in the static case (setting $k=1$ ), with $y_{i,-M}=0$, The first $M$ observations are discarded with $M=49$ and 48 for the $\operatorname{AR}(1)$ and $\operatorname{AR}(2)$ specifications, respectively. For the $\operatorname{AR}(1)$ model, the null hypothesis is defined by $\beta_{i}=\beta=(0.50,0.80,0.90)$, against the alternatives $\beta_{i} \sim \operatorname{IIDU}[\beta-0.1, \beta+0.1)$. For the $\operatorname{AR}(2)$ specification the null is set as $\beta_{1 i}=\beta_{1}=\beta_{2}=(0.25,0.4,0.45)$, against the alternatives, $\beta_{1 i} \sim \operatorname{IIDU}\left[\beta_{2}-0.1, \beta_{2}+0.1\right)$. For these experiments, we consider all the combinations of sample sizes $N$ and $T=20,30,50,100,200$.

The ARDL model is generated as

$$
y_{i t}=\left(1-\beta_{i 1}\right) \alpha_{i}+\beta_{i 1} y_{i t-1}+\beta_{i 2} x_{i t}+\varepsilon_{i t},
$$

where $t=-48, \ldots, 0, \ldots, T, i=1,2, \ldots, N, \varepsilon_{i t}$ 's are generated as before,

$$
x_{i t}=\alpha_{i}\left(1-\rho_{i}\right)+\rho_{i} x_{i, t-1}+\left(1-\rho_{i}^{2}\right)^{1 / 2} v_{i t},
$$

$v_{i t} \sim \operatorname{IIDN}\left(0, \sigma_{i x}^{2}\right)$, and $\alpha_{i}, \sigma_{i}^{2}, \rho_{i}$ and $\sigma_{i x}^{2}$ are drawn randomly across $i$ and then fixed across replications. The initial values are set at $y_{i,-49}=x_{i,-49}=0$, with the first 49 observations discarded. The homogeneity tests considered are (a) $H_{0}: \beta_{i 1}=\beta_{1}$, (b) $H_{0}: \beta_{i 2}=\beta_{2}$, and (c) $H_{0}: \beta_{i 1}=\beta_{1}$ and $\beta_{i 2}=\beta_{2}$ jointly. Different nulls are entertained depending on the nature of the homogeneity tests. Under (a), the null DGP considered is $\beta_{i 1}=\beta_{1}$ and $\beta_{i 2} \sim$ iidU $\left[\beta_{2}-0.1, \beta_{2}+0.1\right.$ ); under (b), $\beta_{i 1} \sim i i d U\left[\beta_{1}-0.05, \beta_{1}+0.05\right]$ and $\beta_{i 2}=\beta_{2}$, and under (c), $\beta_{i 1}=\beta_{1}$ and $\beta_{i 2}=\beta_{2}$. Under the alternative $\beta_{i 1} \sim i i d U\left[\beta_{1}-0.05, \beta_{1}+0.05\right]$ and $\beta_{i 2} \sim i i d U\left[\beta_{2}-0.1, \beta_{2}+0.1\right]$ for all these cases. The parameter values are set at $\beta_{1}=0.5,0.8,0.9$, and $\beta_{2}=1$. To make the experimental results comparable across different values of $\beta_{1}, \sigma_{i x}^{2}$ is set as

$$
\sigma_{i x}^{2}=\left(10-\frac{1}{\left(1-\beta_{1}^{2}\right)}\right)\left(\frac{\sigma_{i}^{2}\left(1-\beta_{1}^{2}\right)\left(1-\beta_{1} \rho_{i}\right)}{\left(1+\beta_{1} \rho_{i}\right) \beta_{2}^{2}}\right) .
$$

so that $R_{i}^{2}=1-\sigma_{i}^{2} / \operatorname{Var}\left(y_{i t}\right)=R^{2}=0.9$. For the subset tests in (a) and (b) the Swamy statistic is computed as (in case (a), for example)

$$
\hat{S}_{1}=\sum_{i=1}^{N}\left(\hat{\beta}_{i 1}-\hat{\beta}_{1, W F E}\right)^{\prime} \frac{\mathbf{y}_{i,-1}^{\prime} \mathbf{M}_{i 2} \mathbf{y}_{i,-1}}{\hat{\sigma}_{i}^{2}}\left(\hat{\beta}_{i 1}-\hat{\beta}_{1, W F E}\right),
$$

where $\mathbf{M}_{i 2}=\mathbf{I}_{T}-\mathbf{Z}_{i 2}\left(\mathbf{Z}_{i 2}^{\prime} \mathbf{Z}_{i 2}\right)^{-1} \mathbf{Z}_{i 2}^{\prime}, \mathbf{Z}_{i 2}=\left(\boldsymbol{\tau}_{T}, \mathbf{x}_{i}\right), \mathbf{x}_{i}=\left(x_{i 1}, x_{i 2}, \ldots, x_{i T},\right)^{\prime}$,

$$
\begin{gathered}
\hat{\beta}_{i 1}=\left(\mathbf{y}_{i,-1}^{\prime} \mathbf{M}_{i 2} \mathbf{y}_{i,-1}\right)^{-1} \mathbf{y}_{i,-1}^{\prime} \mathbf{M}_{i 2} \mathbf{y}_{i}, \\
\hat{\beta}_{1, W F E}=\left(\sum_{i=1}^{N} \frac{\mathbf{y}_{i,-1}^{\prime} \mathbf{M}_{i 2} \mathbf{y}_{i,-1}}{\hat{\sigma}_{i}^{2}}\right)^{-1} \sum_{i=1}^{N} \frac{\mathbf{y}_{i,-1}^{\prime} \mathbf{M}_{i 2} \mathbf{y}_{i}}{\hat{\sigma}_{i}^{2}},
\end{gathered}
$$


and

$$
\hat{\sigma}_{i}^{2}=\frac{\left(\mathbf{y}_{i}-\mathbf{y}_{i,-1} \hat{\beta}_{i 1}\right)^{\prime} \mathbf{M}_{i 2}\left(\mathbf{y}_{i}-\mathbf{y}_{i,-1} \hat{\beta}_{i 1}\right)}{T-3} .
$$

The $\tilde{\Delta}_{1, a d j}$ test statistic is computed using the results in Remark 3 . The Hausman test statistic of $H_{0}: \beta_{i 1}=\beta_{1}$ is defined as

$$
H_{1}=\left(\hat{\beta}_{1, M G}-\tilde{\beta}_{1, W F E}\right)^{2} / v_{H}
$$

where $\hat{\beta}_{1, M G}=N^{-1} \sum_{i=1}^{N} \hat{\beta}_{i 1}$,

$$
\begin{gathered}
\tilde{\beta}_{1, W F E}=\left(\sum_{i=1}^{N} \frac{\mathbf{y}_{i,-1}^{\prime} \mathbf{M}_{i 2} \mathbf{y}_{i,-1}}{\tilde{\sigma}_{i}^{2}}\right)^{-1} \sum_{i=1}^{N} \frac{\mathbf{y}_{i,-1}^{\prime} \mathbf{M}_{i 2} \mathbf{y}_{i}}{\tilde{\sigma}_{i}^{2}} \\
\tilde{\sigma}_{i}^{2}=\frac{\left(\mathbf{y}_{i}-\mathbf{y}_{i,-1} \hat{\beta}_{1, F E}\right)^{\prime} \mathbf{M}_{i 2}\left(\mathbf{y}_{i}-\mathbf{y}_{i,-1} \hat{\beta}_{1, F E}\right)}{T-2}
\end{gathered}
$$

and

$$
v_{H}=N^{-2} \sum_{i=1}^{N} \hat{\sigma}_{i}^{2}\left(\mathbf{y}_{i,-1}^{\prime} \mathbf{M}_{i 2} \mathbf{y}_{i,-1}\right)^{-1}-\left(\sum_{i=1}^{N} \frac{\mathbf{y}_{i,-1}^{\prime} \mathbf{M}_{i 2} \mathbf{y}_{i,-1}}{\tilde{\sigma}_{i}^{2}}\right)^{-1} .
$$

The $\tilde{\Delta}_{1, a d j}$ test is based on a two-sided $N(0,1)$ test. The $H_{1}$ test and the $\hat{S}_{1}$ test are based on $\chi^{2}(1)$ and $\chi^{2}(N-1)$ distributions, respectively. All tests are conducted at $5 \%$ nominal level. The $H_{2}, \hat{S}_{2}$ and $\tilde{\Delta}_{1, a d j}$ test statistics for testing $H_{0}: \beta_{i 2}=\beta_{2}$ are defined in a similar manner.

\subsection{Results}

Size and power of the tests in the case of the experiments with exogenous regressors are summarized in Tables 1-3. Table 1 provides the results for models with one exogenous regressor and normal errors, and give size and power rejection frequencies for a wide range of $N$ and $T$. Table 2 presents the results for models with $k=2,3$ and 4 regressors and normal errors, but for a subset of samples with moderate values of $T=20,30,50$. Table 3 summarizes the same results but when the errors are non-normal.

First, it is clear that in all the experiments the Hausman test, $H$, has the correct size, but, as predicted by our theoretical discussion in Section 2.2, it has no power irrespective of the sample size. On the other hand, the Swamy's $\hat{S}$ test has power, but tends to over-reject when $T$ is small relative to $N$; with the extent of over-rejection diminishing only as $T$ is increased relative to $N$. For example, in the case of $T=10$ and $N=200$ in Table 1 , the empirical size of the $\hat{S}$ test is as much as $82.5 \%$, and only approaches the nominal size of $5 \%$ slowly when $T$ is increased to 200 . The tendency of the $\hat{S}$ test to over-rejection gets accentuated as the number of the regressors is increased or when non-normal errors are considered.

By contrast, the adjusted version of the dispersion test, $\tilde{\Delta}_{a d j}$, has the correct size for all combination of sample sizes, even when $T$ is very small relative to $N$. The size of the test seems also to be very well controlled as the number of regressors is increased to 2 and beyond (in Table 2), or when the errors are generated as draws from a $\chi^{2}(2)$ distribution that represents a significant degree of departure from normality. This last result is particularly welcome and encouraging since the finite sample adjustments are derived under normally distributed errors. ${ }^{7}$ The $\tilde{\Delta}_{a d j}$ test also seems to

\footnotetext{
${ }^{7}$ See Corollary 1 and Remark 2. Also recall that a mean adjustment was not needed in the case of the $\tilde{\Delta}$ test.
} 
have reasonable power properties. Its power rises quite rapidly with both $N$ and $T$, although as predicted by our theoretical derivations in Section 3.2, the power of $\tilde{\Delta}_{\text {adj }}$ test rises much faster with $T$ than with $N$, and comparing the results in Tables 2 and 3 suggest that the power is not much affected by the shape of the error distribution. But the results show that there is a clear tendency for the power to decline with the number of regressors. This is plausible considering that we have controlled the population fit of the regressions so that it remains invariant to the included number of regressors in the panel.

The Monte Carlo results for the dynamic panels are summarized in Tables 4-10. Tables 4 and 5 report the size and power of the $\tilde{\Delta}_{a d j}$ test in the case of the $\operatorname{AR}(1)$ specification, for different values of $\beta=0.5,0.8,0.9$, and for normally and non-normally distributed errors, respectively. Tables 6 and 7 provide the corresponding results for the $\operatorname{AR}(2)$ specification. It is firstly clear that the $H$ test now grossly over-rejects, with the extent of over-rejection in fact rising (rather than falling) with $N$ and $T$. This is in line with our theoretical derivations in Section 2.2 which shows the application of the test to pure dynamic panels fails to satisfy one of the key conditions of the Hausman test. Swamy's $\hat{S}$ test performs reasonably well when $\beta$ is around 0.5 and errors are normally distributed, but begins to show substantial over-rejections when $\beta$ is increased. Similar patterns can also be seen when the results of the $\operatorname{AR}(2)$ specification are considered. It is also interesting that the effects of non-normal errors on the size of the $\hat{S}$ test seems to operate in the opposite direction to the effect of increasing the value of $\beta$ under the null hypothesis. But as predicted by the theory the size distortion of the $\hat{S}$ test declines as $T$ is increased relative to $N$.

Turning to the $\tilde{\Delta}_{a d j}$ test, we first note that its size is very close to the nominal value of $5 \%$ in the case of those experiments with $T \geq N$, which is in line with our asymptotic result for the $\operatorname{AR}(1)$ case that requires $N / T \rightarrow \kappa$ as $(N, T) \stackrel{j}{\rightarrow} \infty$, where $0 \leq \kappa<\infty$. This conclusion seems to be quite robust to the value of the autoregression coefficient, and the shape of the error distribution. In cases where $N>T$, the test outcome crucially depends on the value of $\beta$. It seems to improve when $\beta$ is raised from 0.5 to 0.8 , followed by a substantial deterioration as $\beta$ is further increased to $0.9{ }^{8}$ But overall the $\tilde{\Delta}_{a d j}$ test can not be relied on if $N$ is much larger than $T$. Also as in the case of the experiments with exogenous regressors, the power of the $\tilde{\Delta}_{a d j}$ test rises much more rapidly with $T$ than with $N$. Similar conclusions are obtained if we consider the results for the $\operatorname{AR}(2)$ specification.

The panel ARDL results based on normal errors are summarized in Tables 8-10. Table 8 (resp. Table 9) reports size and power of tests of homogeneity of $\beta_{i 1}\left(\beta_{i 2}\right)$ whilst allowing $\beta_{i 2}\left(\beta_{i 1}\right)$ to vary across $i$. Table 10 reports size and power of homogeneity tests applied to $\beta_{i 1}$ and $\beta_{i 2}$, jointly. Qualitatively, these results are similar to the ones reported above for the AR specifications, although the sub-set test results for $\beta_{i 2}=\beta_{2}$ are generally more satisfactory as compared to the sub-set test results for $\beta_{i 1}$. The Hausman test continues to over-reject, with the extent of over-rejection falling as $T$ rises. The Swamy's test performs well only when $T$ is much larger than $N$. The size of the $\tilde{\Delta}_{a d j}$ test is very close to the nominal value of $5 \%$ in the case of experiments with $T \geq N$. When $N>T$, the size of the $\tilde{\Delta}_{a d j}$ test deteriorates as $\beta_{1}$ increases. The power of all tests decreases as the value of $\beta_{1}$ rises, which largely reflects the nature of the experimental designs. That is, in order to keep $R_{i}^{2}$ fixed as $\beta_{1}$ is increased, the relative variation of the exogenous regressor to the variation of $y_{i, t-1}$ is decreased, which causes a reduction in power of the test.

\footnotetext{
${ }^{8}$ The non-monotone nature of the size of test as a function of $\beta$ can be seen more clearly from additional experiments we have carried out for $\beta=0.3$ and 0.7 which we do not report here.
} 


\section{Concluding Remarks}

In this paper we have developed simple tests of slope homogeneity in linear panel data models where $N$ could be larger than $T$. The proposed tests are based on modifications of Swamy's dispersion statistic and examine the cross section "dispersion" of individual slopes weighted by their relative precision. It is shown that this test is valid when earlier tests based on Hausman (1978) procedure fail to be applicable.

The Monte Carlo evidence shows $\tilde{\Delta}_{a d j}$ test, defined in (29), to have satisfactory size and power properties for all combinations of $N$ and $T$ in the case of panels with exogenous regressors, irrespective of whether the errors are normally distributed or not. In the case of dynamic panels the $\tilde{\Delta}_{a d j}$ test continues to have satisfactory properties for most combinations of $N$ and $T$, so long as the dominant root of the dynamic model, $\beta$, under the null hypothesis is not too close to unity. When $N>T$, and $\beta$ is around 0.9 or more, the $\tilde{\Delta}_{a d j}$ test tends to over-reject, which is primarily due to the fact that for large values of $N / T$ the small sample bias of the fixed effects estimator of $\beta$ can be substantial. Our preliminary investigation suggests that in such cases a bootstrapped version of the test that makes use of the bias-corrected FE estimator proposed in Hahn and Kuersteiner (2002) could be more appropriate.

Another important extension of the tests developed in this paper is to consider testing slope homogeneity in panel data models with multi-factor error structures recently examined in Pesaran (2006). This is, however, beyond the scope of the present paper. 


\section{A Mathematical Appendix}

\section{A.1 Preliminary Results}

Lemma 1 Let $\boldsymbol{v} \sim \operatorname{IIDN}\left(\mathbf{0}, \mathbf{I}_{T}\right)$, and $\mathbf{A}$ be a $T \times T$ positive semi-definite symmetric matrix of rank $m \leq T$. Then the $r^{\text {th }}$ moment of the inverse of $\boldsymbol{v}^{\prime} \mathbf{A} \boldsymbol{v}$ exists if $m>2 r$.

Proof. See Smith (1988).

Corollary 2 Let $\mathbf{A}_{1}$ and $\mathbf{A}_{2}$ be $T \times T$ positive semi-definite symmetric matrices of rank $m_{1}$ and $m_{2}$, respectively. Then the $r^{\text {th }}$ moment of the inverse of $\left(\boldsymbol{v}^{\prime} \mathbf{A}_{1} \boldsymbol{v}\right)\left(\boldsymbol{v}^{\prime} \mathbf{A}_{2} \boldsymbol{v}\right)$ exists if $m_{1}>4 r$ and $m_{2}>4 r$.

Proof. The result is immediate from Lemma 1 using the Cauchy-Schwarz inequality.

Lemma 2 Let

$$
\boldsymbol{\xi}_{i T}=T^{-1 / 2} \mathbf{X}_{i}^{\prime} \mathbf{M}_{\tau} \boldsymbol{\varepsilon}_{i}, \boldsymbol{\xi}_{N T}=N^{-1 / 2} \sum_{i=1}^{N} \boldsymbol{\xi}_{i T} .
$$

Then, under Assumptions 1 and 2, for each $i$,

$$
\boldsymbol{\xi}_{i T} \rightarrow{ }_{d} N\left(\mathbf{0}, \sigma_{i}^{2} \mathbf{Q}_{i}\right) \text {, as } T \rightarrow \infty,
$$

and

$$
\boldsymbol{\xi}_{N T} \rightarrow_{d} N\left(\mathbf{0}, \mathbf{Q}_{\sigma}\right) \text {, as }(N, T) \stackrel{j}{\rightarrow} \infty,
$$

where $\mathbf{Q}_{\sigma}=\lim _{N \rightarrow \infty} N^{-1} \sum_{i=1}^{N} \sigma_{i}^{2} \mathbf{Q}_{i}$, where $\mathbf{Q}_{i}$ is defined by Assumption $2(i)$.

Proof. The first result follows from Assumption 1 by familiar central limit theorems used for the classical linear regression models. The second result follows since $\varepsilon_{1}, \varepsilon_{2}, \ldots, \varepsilon_{N}$ are assumed to be independently distributed, and $E\left\|\mathbf{Q}_{i T}\right\|<K$ for all $i$. See Lemma 4 in Pesaran (2006).

Lemma 3 Suppose that $\mathbf{D}$ is a $p \times p$ stochastic matrix; non-singular with probability one, such that $\mathbf{D}=O_{p}(1), E(\mathbf{D})=O(1)$, and $\mathbf{D}-E(\mathbf{D})=O_{p}\left(T^{-1 / 2}\right)$. Then,

$$
\mathbf{D}^{-1}=[E(\mathbf{D})]^{-1}+O_{p}\left(T^{-1 / 2}\right) .
$$

Proof. See Kiviet and Phillips (1994, Lemma 2).

Lemma 4 Let $\boldsymbol{\Phi}$ be a $T \times T$ symmetric matrix and $\boldsymbol{\Gamma}$ a positive definite $T \times T$ matrix, and suppose that $\boldsymbol{v} \sim \operatorname{IID}\left(\mathbf{0}, \mathbf{I}_{T}\right)$, where $\boldsymbol{v}=\left(v_{1}, v_{2}, \ldots, v_{T}\right)^{\prime}$. Denote the $p^{\text {th }}$ cumulant of $\boldsymbol{v}^{\prime} \boldsymbol{\Gamma} \boldsymbol{v}$ by $\kappa_{p}$, and the $\gamma=1+m$ order, $\delta=r+m$ degree generalized cumulant of $\left(\boldsymbol{v}^{\prime} \boldsymbol{\Phi} \boldsymbol{v}\right)^{r}\left(\boldsymbol{v}^{\prime} \boldsymbol{\Gamma} \boldsymbol{v}\right)$ by $\kappa_{r m}$ and assume that the following conditions hold

- Condition 1 For $p=1,2, \ldots, \kappa_{p}=O(T)$.

- Condition 2 For $r=1,2, \ldots, \kappa_{r 0}=E\left(\boldsymbol{v}^{\prime} \boldsymbol{\Phi} \boldsymbol{v}\right)^{r}=O\left(T^{r}\right)$. 
- Condition 3 For $r, m=1,2, \ldots, \kappa_{r m}=O\left(T^{\ell}\right)$, with $\ell \leq r$.

Then the Laplace approximate expansion for the $r^{\text {th }}$ moment of $\boldsymbol{v}^{\prime} \boldsymbol{\Phi} \boldsymbol{v} / \boldsymbol{v}^{\prime} \boldsymbol{\Gamma} \boldsymbol{v}$ is given by

$$
E\left(\frac{\boldsymbol{v}^{\prime} \boldsymbol{\Phi} \boldsymbol{v}}{\boldsymbol{v}^{\prime} \boldsymbol{\Gamma} \boldsymbol{v}}\right)^{r}=\frac{E\left[\left(\boldsymbol{v}^{\prime} \boldsymbol{\Phi} \boldsymbol{v}\right)^{r}\right]}{\left[E\left(\boldsymbol{v}^{\prime} \boldsymbol{\Gamma} \boldsymbol{v}\right)\right]^{r}}+\varphi_{1 T}+\varphi_{2 T}+O\left(T^{-3}\right)
$$

where

$$
\begin{gathered}
\varphi_{1 T}=\frac{r(r+1)}{2}\left[\frac{E\left[\left(\boldsymbol{v}^{\prime} \boldsymbol{\Phi} \boldsymbol{v}\right)^{r}\right] \kappa_{2}}{\left[E\left(\boldsymbol{v}^{\prime} \boldsymbol{\Gamma} \boldsymbol{v}\right)\right]^{r+2}}\right]-r\left[\frac{\kappa_{r 1}}{\left[E\left(\boldsymbol{v}^{\prime} \boldsymbol{\Gamma} \boldsymbol{v}\right)\right]^{r+1}}\right], \\
\varphi_{2 T}=\frac{r(r+1)}{2}\left[\frac{\kappa_{r 2}}{\left[E\left(\boldsymbol{v}^{\prime} \boldsymbol{\Gamma} \boldsymbol{v}\right)\right]^{r+2}}\right]-\frac{r(r+1)(r+2)}{2}\left[\frac{3 E\left[\left(\boldsymbol{v}^{\prime} \boldsymbol{\Phi} \boldsymbol{v}\right)^{r}\right] \kappa_{3}+\kappa_{r 1} \kappa_{2}}{\left[E\left(\boldsymbol{v}^{\prime} \boldsymbol{\Gamma} \boldsymbol{v}\right)\right]^{r+3}}\right] \\
+\frac{r(r+1)(r+2)(r+3)}{8}\left[\frac{E\left[\left(\boldsymbol{v}^{\prime} \boldsymbol{\Phi} \boldsymbol{v}\right)^{r}\right] \kappa_{2}^{2}}{\left[E\left(\boldsymbol{v}^{\prime} \boldsymbol{\Gamma} \boldsymbol{v}\right)\right]^{r+4}}\right],
\end{gathered}
$$

and

$$
\begin{gathered}
\kappa_{r 1}=E\left[\left(\boldsymbol{v}^{\prime} \boldsymbol{\Phi} \boldsymbol{v}\right)^{r} \boldsymbol{v}^{\prime} \boldsymbol{\Gamma} \boldsymbol{v}\right]-E\left[\left(\boldsymbol{v}^{\prime} \boldsymbol{\Phi} \boldsymbol{v}\right)^{r}\right] E\left(\boldsymbol{v}^{\prime} \boldsymbol{\Gamma} \boldsymbol{v}\right) \\
\kappa_{r 2}=E\left[\left(\boldsymbol{v}^{\prime} \boldsymbol{\Phi} \boldsymbol{v}\right)^{r}\left(\boldsymbol{v}^{\prime} \boldsymbol{\Gamma} \boldsymbol{v}\right)^{2}\right]-2 E\left(\boldsymbol{v}^{\prime} \boldsymbol{\Gamma} \boldsymbol{v}\right) E\left[\left(\boldsymbol{v}^{\prime} \boldsymbol{\Phi} \boldsymbol{v}\right)^{r}\left(\boldsymbol{v}^{\prime} \boldsymbol{\Gamma} \boldsymbol{v}\right)\right] \\
-E\left[\left(\boldsymbol{v}^{\prime} \boldsymbol{\Gamma} \boldsymbol{v}\right)^{2}\right] E\left[\left(\boldsymbol{v}^{\prime} \boldsymbol{\Phi} \boldsymbol{v}\right)^{r}\right]+2\left[\left(E\left(\boldsymbol{v}^{\prime} \boldsymbol{\Gamma} \boldsymbol{v}\right)\right]^{2} E\left[\left(\boldsymbol{v}^{\prime} \boldsymbol{\Phi} \boldsymbol{v}\right)^{r}\right]\right.
\end{gathered}
$$

Proof. Lieberman (1994).

\section{A.2 Proof of Theorem 1}

For the Swamy's version of the dispersion test, under $H_{0}$ we have

$$
\left(\hat{\boldsymbol{\beta}}_{i}-\hat{\boldsymbol{\beta}}_{W F E}\right)=T^{-1 / 2} \mathbf{Q}_{i T}^{-1} \boldsymbol{\xi}_{i T}-T^{-1 / 2} N^{-1 / 2}\left(N^{-1} \sum_{i=1}^{N} \hat{\sigma}_{i}^{-2} \mathbf{Q}_{i T}\right)^{-1}\left(N^{-1 / 2} \sum_{i=1}^{N} \hat{\sigma}_{i}^{-2} \boldsymbol{\xi}_{i T}\right),
$$

where $\mathbf{Q}_{i T}$ and $\boldsymbol{\xi}_{i T}$ are defined by (16) and (A.1), respectively. Using this result in (11) it is easily seen that

$$
\frac{1}{N} \hat{S}=\frac{1}{N} \sum_{i=1}^{N} \frac{\boldsymbol{\xi}_{i T}^{\prime} \mathbf{Q}_{i T}^{-1} \boldsymbol{\xi}_{i T}}{\hat{\sigma}_{i}^{2}}-\frac{1}{N}\left(\frac{\sum_{i=1}^{N} \hat{\sigma}_{i}^{-2} \boldsymbol{\xi}_{i T}}{\sqrt{N}}\right)^{\prime}\left(\frac{\sum_{i=1}^{N} \hat{\sigma}_{i}^{-2} \mathbf{Q}_{i T}}{N}\right)^{-1}\left(\frac{\sum_{i=1}^{N} \hat{\sigma}_{i}^{-2} \boldsymbol{\xi}_{i T}}{\sqrt{N}}\right) .
$$

We first note that (by Cauchy-Schwarz inequality)

$$
E\left\|T^{1 / 2}\left(\hat{\sigma}_{i}^{-2}-\sigma_{i}^{-2}\right) \boldsymbol{\xi}_{i T}\right\| \leq \sqrt{E\left[T\left(\hat{\sigma}_{i}^{-2}-\sigma_{i}^{-2}\right)^{2}\right]} \sqrt{E\left\|T^{-1 / 2} \mathbf{X}_{i}^{\prime} \mathbf{M}_{\tau} \boldsymbol{\varepsilon}_{i}\right\|^{2}}
$$

and by Assumptions 1-2

$$
E\left\|T^{-1 / 2} \mathbf{X}_{i}^{\prime} \mathbf{M}_{\tau} \varepsilon_{i}\right\|^{2} \leq \sigma_{\max }^{2} \operatorname{Tr}\left(\mathbf{Q}_{i}\right)<K, \text { for all } i .
$$

Also

$$
E\left[T\left(\hat{\sigma}_{i}^{-2}-\sigma_{i}^{-2}\right)^{2}\right]=T E\left(\frac{\sigma_{i}^{2}-\hat{\sigma}_{i}^{2}}{\sigma_{i}^{2} \hat{\sigma}_{i}^{2}}\right)^{2} \leq \frac{1}{\sigma_{\min }^{4}} \sqrt{T^{2} E\left(1-\frac{\hat{\sigma}_{i}^{2}}{\sigma_{i}^{2}}\right)^{4}} \sqrt{E\left(\frac{\sigma_{i}^{2}}{\hat{\sigma}_{i}^{2}}\right)^{4}} .
$$


But by Assumptions 1(i) and 3

$$
E\left(\frac{\sigma_{i}^{2}}{\hat{\sigma}_{i}^{2}}\right)^{4}=E\left(\frac{T-k-1}{\boldsymbol{v}_{i}^{\prime} \mathbf{M}_{i} \boldsymbol{v}_{i}}\right)^{4}<K
$$

and by using the results of Bao and Ullah (2006) for the 4th order moments of the quadratic form of non-normal errors, after some tedious algebra, we obtain ${ }^{9}$

$$
E\left(1-\frac{\hat{\sigma}_{i}^{2}}{\sigma_{i}^{2}}\right)^{4}=O\left(T^{-2}\right)
$$

Hence

$$
E\left\|T^{1 / 2}\left(\hat{\sigma}_{i}^{-2}-\sigma_{i}^{-2}\right) \boldsymbol{\xi}_{i T}\right\|=O(1)
$$

and

$$
E\left\|\frac{\sum_{i=1}^{N} T^{1 / 2}\left(\hat{\sigma}_{i}^{-2}-\sigma_{i}^{-2}\right) \boldsymbol{\xi}_{i T}}{N}\right\| \leq \frac{\sum_{i=1}^{N} E\left\|T^{1 / 2}\left(\hat{\sigma}_{i}^{-2}-\sigma_{i}^{-2}\right) \boldsymbol{\xi}_{i T}\right\|}{N}=O(1) .
$$

Thus, since $\hat{\sigma}_{i}^{2}-\sigma_{i}^{2}=O_{p}\left(T^{-1 / 2}\right)$, by Lemma 3 , and noting that $E\left\|T^{1 / 2}\left(\hat{\sigma}_{i}^{-2}-\sigma_{i}^{-2}\right) \boldsymbol{\xi}_{i T}\right\|<K$ uniformly over $i$, we conclude that

$$
\frac{\sum_{i=1}^{N} \hat{\sigma}_{i}^{-2} \boldsymbol{\xi}_{i T}}{N}=\frac{\sum_{i=1}^{N} \sigma_{i}^{-2} \boldsymbol{\xi}_{i T}}{N}+O_{p}\left(T^{-1 / 2}\right)
$$

Also as $(N, T) \stackrel{j}{\rightarrow} \infty$

$$
\frac{\sum_{i=1}^{N} \sigma_{i}^{-2} \boldsymbol{\xi}_{i T}}{\sqrt{N}} \rightarrow_{d} N\left(\mathbf{0}, \mathbf{Q}_{*}\right),
$$

where $\mathbf{Q}_{*}=\lim _{N \rightarrow \infty} N^{-1} \sum_{i=1}^{N} \sigma_{i}^{-2} \mathbf{Q}_{i}$, and $E\left\|\mathbf{Q}_{*}\right\|<K{ }^{10}$ Hence

$$
\frac{\sum_{i=1}^{N} \hat{\sigma}_{i}^{-2} \boldsymbol{\xi}_{i T}}{\sqrt{N}}=O_{p}(1)+O_{p}\left(\sqrt{\frac{N}{T}}\right) .
$$

Similarly

and by Lemma 3

$$
\frac{\sum_{i=1}^{N} \hat{\sigma}_{i}^{-2} \mathbf{Q}_{i T}}{N}=\frac{\sum_{i=1}^{N} \sigma_{i}^{-2} \mathbf{Q}_{i T}}{N}+O_{p}\left(T^{-1 / 2}\right)
$$

$$
\left(\frac{\sum_{i=1}^{N} \hat{\sigma}_{i}^{-2} \mathbf{Q}_{i T}}{N}\right)^{-1}=\left(\frac{\sum_{i=1}^{N} \sigma_{i}^{-2} \mathbf{Q}_{i}}{N}\right)^{-1}+O_{p}\left(T^{-1 / 2}\right) .
$$

Using the above results we now have

$$
\begin{aligned}
& \left(\frac{\sum_{i=1}^{N} \hat{\sigma}_{i}^{-2} \boldsymbol{\xi}_{i T}}{\sqrt{N}}\right)^{\prime}\left(\frac{\sum_{i=1}^{N} \hat{\sigma}_{i}^{-2} \mathbf{Q}_{i T}}{N}\right)^{-1}\left(\frac{\sum_{i=1}^{N} \hat{\sigma}_{i}^{-2} \boldsymbol{\xi}_{i T}}{\sqrt{N}}\right) \\
= & O_{p}(1)+O_{p}\left(T^{-1 / 2}\right)+O_{p}\left(\sqrt{\frac{N}{T}}\right),
\end{aligned}
$$

\footnotetext{
${ }^{9}$ Details are provided in a supplement and are available upon request.

${ }^{10}$ Note that

by assumption.

$$
E\left\|N^{-1} \sum_{i=1}^{N} \sigma_{i}^{-2} \mathbf{Q}_{i}\right\| \leq \sigma_{\min }^{-2} N^{-1} \sum_{i=1}^{N} E\left\|\mathbf{Q}_{i}\right\|<K,
$$
}


and

$$
\frac{1}{N} \hat{S}=\frac{1}{N} \sum_{i=1}^{N} \frac{\boldsymbol{\xi}_{i T}^{\prime} \mathbf{Q}_{i T}^{-1} \boldsymbol{\xi}_{i T}}{\hat{\sigma}_{i}^{2}}+O_{p}\left(\frac{1}{N}\right)+O_{p}\left(\sqrt{\frac{1}{N T}}\right)
$$

Also

$$
\frac{\boldsymbol{\xi}_{i T}^{\prime} \mathbf{Q}_{i T}^{-1} \boldsymbol{\xi}_{i T}}{\hat{\sigma}_{i}^{2}}=\left(\frac{\boldsymbol{\varepsilon}_{i}^{\prime} \mathbf{M}_{i} \boldsymbol{\varepsilon}_{i}}{T-k-1}\right)^{-1} \boldsymbol{\xi}_{i T}^{\prime} \mathbf{Q}_{i T}^{-1} \boldsymbol{\xi}_{i T}=\frac{(T-k-1) \boldsymbol{v}_{i}^{\prime} \mathbf{P}_{i} \boldsymbol{v}_{i}}{\boldsymbol{v}_{i}^{\prime} \mathbf{M}_{i} \boldsymbol{v}_{i}}
$$

Hence

$$
N^{-1 / 2} \hat{S}=N^{-1 / 2} \sum_{i=1}^{N} \hat{z}_{i T}+O_{p}\left(N^{-1 / 2}\right)+O_{p}\left(T^{-1 / 2}\right)
$$

as required.

Similar results also hold for the modified version of Swamy's statistic, $\tilde{S}$. Under $H_{0}$ we have

$$
\frac{1}{N} \tilde{S}=\frac{1}{N} \sum_{i=1}^{N} \frac{\boldsymbol{\xi}_{i T}^{\prime} \mathbf{Q}_{i T}^{-1} \boldsymbol{\xi}_{i T}}{\tilde{\sigma}_{i}^{2}}-\frac{1}{N}\left(\frac{\sum_{i=1}^{N} \tilde{\sigma}_{i}^{-2} \boldsymbol{\xi}_{i T}}{\sqrt{N}}\right)^{\prime}\left(\frac{\sum_{i=1}^{N} \tilde{\sigma}_{i}^{-2} \mathbf{Q}_{i T}}{N}\right)^{-1}\left(\frac{\sum_{i=1}^{N} \tilde{\sigma}_{i}^{-2} \boldsymbol{\xi}_{i T}}{\sqrt{N}}\right) .
$$

Using (14), and after some algebra (under $H_{0}$ ) we obtain

$$
\tilde{\sigma}_{i}^{2}=\frac{\boldsymbol{\varepsilon}_{i}^{\prime} \mathbf{M}_{\tau} \varepsilon_{i}}{T-1}+\frac{1}{N(T-1)} \boldsymbol{\xi}_{N T}^{\prime} \mathbf{Q}_{N T}^{-1} \mathbf{Q}_{i T} \mathbf{Q}_{N T}^{-1} \boldsymbol{\xi}_{N T}+\frac{2}{\sqrt{N}(T-1)} \boldsymbol{\xi}_{N T}^{\prime} \mathbf{Q}_{N T}^{-1} \boldsymbol{\xi}_{i T} .
$$

Also under Assumptions 1-2, and using Lemma 2 it is easily seen that

$$
\boldsymbol{\xi}_{N T}^{\prime} \mathbf{Q}_{N T}^{-1} \mathbf{Q}_{i T} \mathbf{Q}_{N T}^{-1} \boldsymbol{\xi}_{N T}=O_{p}(1) \text { and } \boldsymbol{\xi}_{N T}^{\prime} \mathbf{Q}_{N T}^{-1} \boldsymbol{\xi}_{i T}=O_{p}(1)
$$

Therefore

$$
\tilde{\sigma}_{i}^{2}=\frac{\varepsilon_{i}^{\prime} \mathbf{M}_{\tau} \varepsilon_{i}}{T-1}+O_{p}\left(N^{-1 / 2} T^{-1}\right),
$$

and following a similar line of reasoning as above we have

$$
\begin{aligned}
N^{-1 / 2} \sum_{i=1}^{N} \tilde{\sigma}_{i}^{-2} \boldsymbol{\xi}_{i T} & =N^{-1 / 2} \sum_{i=1}^{N} \sigma_{i}^{-2} \boldsymbol{\xi}_{i T}+O_{p}\left(T^{-1 / 2}\right) \\
N^{-1} \sum_{i=1}^{N} \tilde{\sigma}_{i}^{-2} \mathbf{Q}_{i T} & =N^{-1} \sum_{i=1}^{N} \sigma_{i}^{-2} \mathbf{Q}_{i T}+O_{p}\left(T^{-1 / 2}\right)
\end{aligned}
$$

and

$$
\frac{1}{N} \sum_{i=1}^{N} \frac{\boldsymbol{\xi}_{i T}^{\prime} \mathbf{Q}_{i T}^{-1} \boldsymbol{\xi}_{i T}}{\tilde{\sigma}_{i}^{2}}=\frac{1}{N} \sum_{i=1}^{N} \frac{(T-1) \boldsymbol{\xi}_{i T}^{\prime} \mathbf{Q}_{i T}^{-1} \boldsymbol{\xi}_{i T}}{\varepsilon_{i}^{\prime} \mathbf{M}_{\tau} \varepsilon_{i}}+O_{p}\left(N^{-1 / 2} T^{-1}\right)
$$

Hence, using these results in (A.14) we obtain

$$
N^{-1 / 2} \tilde{S}=N^{-1 / 2} \sum_{i=1}^{N} \tilde{z}_{i T}+O_{p}\left(T^{-1 / 2}\right)+O_{p}\left(N^{-1 / 2}\right),
$$

where $\tilde{z}_{i T}$ is defined by (22). 


\section{A.3 Moments of $\hat{z}_{i T}$ and $\tilde{z}_{i T}$ under Nonnormality}

To obtain the first two moments of $\hat{z}_{i T}$, defined by (22), under nonnormal error we exploit a slightly extended version of Lieberman's results, reproduced in Lemma 4 for convenience, that allows $\boldsymbol{\Gamma}$ defined in that Lemma to be a semi-positive definite matrix. In the case of our application, $\boldsymbol{\Gamma}$ is defined by the symmetric, idempotent matrix, $\mathbf{M}_{i}$, with $\operatorname{rank} T-k-1$, and it is easily seen that $\operatorname{Pr}\left[\boldsymbol{v}_{i}^{\prime} \mathbf{M}_{i} \boldsymbol{v}_{i}=0\right]=0 .{ }^{11}$ Also it is possible to show that Conditions 1, 2 and 3 of Lemma 4 are satisfied. ${ }^{12}$ In particular, it is possible to show that $\varphi_{i 1 T}$, defined by by (A.5) for $\hat{z}_{i T}$, is $O\left(T^{-1}\right)$ for all $i$, and using (A.4), we have

$$
E\left(\hat{z}_{i T}\right)=\frac{(T-k-1) E\left(\boldsymbol{v}_{i}^{\prime} \mathbf{P}_{i} \boldsymbol{v}_{i}\right)}{E\left(\boldsymbol{v}_{i}^{\prime} \mathbf{M}_{i} \boldsymbol{v}_{i}\right)}+\varphi_{i 1 T}+O\left(T^{-2}\right)
$$

uniformly over $i$. Now using results from Ullah (2004) and Bao and Ullah (2006) on the moments of quadratic forms in nonnormal variables ${ }^{13}$ we have

$$
\varphi_{i 1 T}=\frac{2(T-k-1)+\gamma_{i 2} \operatorname{Tr}\left(\mathbf{M}_{i} \odot \mathbf{M}_{i}\right)}{(T-k-1)^{2}}-\frac{\gamma_{i 2} \operatorname{Tr}\left(\mathbf{M}_{i} \odot \mathbf{P}_{i}\right)}{(T-k-1)}
$$

where $\gamma_{i 2}$ is the Pearson's measure of kurtosis, which is zero when $v_{i t}$ is standard normal, and $\odot$ is Hadamard product. Since $\left|\gamma_{i 2}\right|<K$ by Assumption $1(\mathrm{iii}), \operatorname{tr}\left(\mathbf{M}_{i} \odot \mathbf{M}_{i}\right) \leq(T-k-1)$, and $0 \leq \operatorname{tr}\left(\mathbf{M}_{i} \odot \mathbf{P}_{i}\right) \leq k,{ }^{14}$ then it follows that $\varphi_{i 1 T}=O\left(T^{-1}\right)$, uniformly over $i$. Hence, using (A.17) we have $E\left(\hat{z}_{i T}\right)=k+O\left(T^{-1}\right)$ uniformly over $i$. Similarly

$$
\begin{aligned}
\operatorname{Var}\left(\hat{z}_{i T}\right) & =\frac{(T-k-1)^{2} E\left[\left(\boldsymbol{v}_{i}^{\prime} \mathbf{P}_{i} \boldsymbol{v}_{i}\right)^{2}\right]}{\left[E\left(\boldsymbol{v}_{i}^{\prime} \mathbf{M}_{i} \boldsymbol{v}_{i}\right)\right]^{2}}-k^{2}+O\left(T^{-1}\right) \\
& =2 k+O\left(T^{-1}\right)
\end{aligned}
$$

uniformly over $i$.

Consider now

$$
\tilde{z}_{i T}=\frac{(T-1) \boldsymbol{v}_{i}^{\prime} \mathbf{P}_{i} \boldsymbol{v}_{i}}{\boldsymbol{v}_{i}^{\prime} \mathbf{M}_{\tau} \boldsymbol{v}_{i}}
$$

and note that $\operatorname{Pr}\left[\boldsymbol{v}_{i}^{\prime} \mathbf{M}_{\tau} \boldsymbol{v}_{i}=0\right]=0$ for $T-1>0$, and Lemma 4 is applicable to $\tilde{z}_{i T}$ so long as $T>1$. Also, it is easily verified that

$$
\frac{(T-1) E\left(\boldsymbol{v}_{i}^{\prime} \mathbf{P}_{i} \boldsymbol{v}_{i}\right)}{E\left(\boldsymbol{v}_{i}^{\prime} \mathbf{M}_{\tau} \boldsymbol{v}_{i}\right)}=k,
$$

and the Conditions 1, 2 and 3 in Lemma 4 are satisfied for the first two moments of $\tilde{z}_{i T}$. Also after some algebra (details of which are available on request) it can be established that $\varphi_{i 1 T}=0$ and $\varphi_{i 2 T}=O\left(T^{-2}\right)$ uniformly over $i$ (See (A.5) and (A.6).). Therefore,

$$
E\left(\tilde{z}_{i T}\right)=k+O\left(T^{-2}\right), \text { uniformly over } i .
$$

Similarly, using (A.4) we have

$$
\operatorname{Var}\left(\tilde{z}_{i T}\right)=2 k+O\left(T^{-1}\right)
$$

uniformly over $i$.

\footnotetext{
${ }^{11}$ We are grateful to Dr. Lieberman for this extension of his published results through a private communication.

${ }^{12}$ The proof of the conditions 1-3 of Lemma 4 can be obtained from the authors on request.

${ }^{13}$ Details are provided in a supplement to the paper that can be obtained from the authors on request.

${ }^{14}$ Note that all diagonal elements of $\mathbf{M}_{i}$ and $\mathbf{P}_{i}$ are non-negative and bounded by unity.
} 


\section{A.4 Proof of Theorem 2}

Using (20), first note that

$$
\hat{\Delta}=\frac{1}{\sqrt{N}} \sum_{i=1}^{N}\left(\frac{\hat{z}_{i T}-k}{\sqrt{2 k}}\right)+O_{p}\left(T^{-1 / 2}\right)+O_{p}\left(N^{-1 / 2}\right),
$$

and write

$$
\frac{1}{N} \sum_{i=1}^{N}\left(\frac{\hat{z}_{i T}-k}{\sqrt{2 k}}\right)=\frac{1}{N} \sum_{i=1}^{N}\left(\frac{\hat{z}_{i T}-E\left(\hat{z}_{i T}\right)}{\sqrt{2 k}}\right)+\frac{1}{N} \sum_{i=1}^{N}\left(\frac{E\left(\hat{z}_{i T}\right)-k}{\sqrt{2 k}}\right) .
$$

Using $E\left(\hat{z}_{i T}-k\right)=O\left(T^{-1}\right)$, established by (A.17),

$$
\frac{1}{N} \sum_{i=1}^{N}\left(\frac{\hat{z}_{i T}-k}{\sqrt{2 k}}\right)=\frac{1}{N} \sum_{i=1}^{N}\left(\frac{\hat{z}_{i T}-E\left(\hat{z}_{i T}\right)}{\sqrt{2 k}}\right)+O\left(T^{-1}\right) .
$$

Hence

$$
\hat{\Delta}=\frac{1}{\sqrt{N}} \sum_{i=1}^{N}\left(\frac{\hat{z}_{i T}-E\left(\hat{z}_{i T}\right)}{\sqrt{2 k}}\right)+O\left(N^{1 / 2} T^{-1}\right)+O_{p}\left(T^{-1 / 2}\right)+O_{p}\left(N^{-1 / 2}\right) .
$$

Under our assumptions, $\hat{z}_{i T}$ is independently but not necessarily identically distributed across $i$. But by Cauchy-Schwarz inequality

$$
E\left|\hat{z}_{i T}\right|^{2+\epsilon / 2}<\sqrt{E\left(\boldsymbol{v}_{i}^{\prime} \mathbf{P}_{i} \boldsymbol{v}_{i}\right)^{4+\epsilon}} \sqrt{E\left(\frac{T-k-1}{\boldsymbol{v}_{i}^{\prime} \mathbf{M}_{i} \boldsymbol{v}_{i}}\right)^{4+\epsilon}}
$$

for some small positive constant $\epsilon$, and under Assumptions 1 and $3 E\left|\hat{z}_{i T}\right|^{2+\epsilon / 2}<K<\infty$, and hence the Lindberg-Feller Central Limit Theorem is directly applicable to the first term of (A.21), (White (2001, Section 5.2)), and

$$
\frac{1}{\sqrt{N}} \sum_{i=1}^{N}\left(\frac{\hat{z}_{i T}-E\left(\hat{z}_{i T}\right)}{\sqrt{2 k}}\right) \rightarrow_{d} N\left(0, \hat{g}^{2}\right)
$$

where

$$
\hat{g}^{2}=\lim _{N \rightarrow \infty} N^{-1} \sum_{i=1}^{N}\left(\frac{\operatorname{Var}\left(\hat{z}_{i T}\right)}{2 k}\right) .
$$

But since $\operatorname{Var}\left(\hat{z}_{i T}\right)=2 k+O\left(T^{-1}\right)$ for all $i$, then $\hat{g}^{2}=1+O\left(T^{-1}\right)$ and using the above results we have

$$
\hat{\Delta} \rightarrow{ }_{d} N(0,1) \text { as }(N, T) \stackrel{j}{\rightarrow} \infty, \text { so long as } \sqrt{N} / T \rightarrow 0 .
$$

Similarly

$$
\tilde{\Delta}=\frac{1}{\sqrt{N}} \sum_{i=1}^{N}\left(\frac{\tilde{z}_{i T}-k}{\sqrt{2 k}}\right)+O_{p}\left(T^{-1 / 2}\right)+O_{p}\left(N^{-1 / 2}\right),
$$

and as before we have

$$
\frac{1}{N} \sum_{i=1}^{N}\left(\frac{\tilde{z}_{i T}-k}{\sqrt{2 k}}\right)=\frac{1}{N} \sum_{i=1}^{N}\left(\frac{\tilde{z}_{i T}-E\left(\tilde{z}_{i T}\right)}{\sqrt{2 k}}\right)+\frac{1}{N} \sum_{i=1}^{N}\left(\frac{E\left(\tilde{z}_{i T}\right)-k}{\sqrt{2 k}}\right) .
$$


However, using (A.19), $E\left(\tilde{z}_{i T}\right)=k+O\left(T^{-2}\right)$

$$
\frac{1}{N} \sum_{i=1}^{N}\left(\frac{\tilde{z}_{i T}-k}{\sqrt{2 k}}\right)=\frac{1}{N} \sum_{i=1}^{N}\left(\frac{\tilde{z}_{i T}-E\left(\tilde{z}_{i T}\right)}{\sqrt{2 k}}\right)+O\left(T^{-2}\right)
$$

and

$$
\tilde{\Delta}=\frac{1}{\sqrt{N}} \sum_{i=1}^{N}\left(\frac{\tilde{z}_{i T}-E\left(\tilde{z}_{i T}\right)}{\sqrt{2 k}}\right)+O\left(N^{1 / 2} T^{-2}\right)+O_{p}\left(T^{-1 / 2}\right)+O_{p}\left(N^{-1 / 2}\right) .
$$

As in the case of $\hat{\Delta}$,

$$
\frac{1}{\sqrt{N}} \sum_{i=1}^{N}\left(\frac{\tilde{z}_{i T}-E\left(\tilde{z}_{i T}\right)}{\sqrt{2 k}}\right) \rightarrow{ }_{d} N\left(0, \tilde{g}^{2}\right)
$$

where

$$
\tilde{g}^{2}=\lim _{N \rightarrow \infty} N^{-1} \sum_{i=1}^{N}\left(\frac{\operatorname{Var}\left(\tilde{z}_{i T}\right)}{2 k}\right),
$$

with $\operatorname{Var}\left(\tilde{z}_{i T}\right) / 2 k=1+O\left(T^{-1}\right)$. Therefore,

$$
\tilde{\Delta} \rightarrow{ }_{d} N(0,1) \text { as }(N, T) \stackrel{j}{\rightarrow} \infty \text { so long as } \sqrt{N} / T^{2} \rightarrow 0 .
$$

\section{A.5 Proof of Corollary 1}

\section{A.5.1 Moments of $\hat{z}_{i T}$ and $\tilde{z}_{i T}$ under Normality}

For the moments of $\hat{z}_{i T}=\varepsilon_{i}^{\prime} \mathbf{P}_{i} \varepsilon_{i} /\left[\varepsilon_{i}^{\prime} \mathbf{M}_{i} \varepsilon_{i} /(T-k-1)\right]$, where $\mathbf{P}_{i}$ and $\mathbf{M}_{i}$ are defined by (18) and (19) respectively, noting $\mathbf{P}_{i} \mathbf{M}_{i}=\mathbf{M}_{i} \mathbf{P}_{i}=\mathbf{0}, \boldsymbol{\varepsilon}_{i}^{\prime} \mathbf{P}_{i} \varepsilon_{i} / \sigma_{i}^{2} \sim \chi^{2}(k)$ and $\boldsymbol{\varepsilon}_{i}^{\prime} \mathbf{M}_{i} \varepsilon_{i} / \sigma_{i}^{2} \sim \chi^{2}(T-k-1)$, which are independent of each other,

$$
\frac{\boldsymbol{\varepsilon}_{i}^{\prime} \mathbf{P}_{i} \boldsymbol{\varepsilon}_{i} / k}{\boldsymbol{\varepsilon}_{i}^{\prime} \mathbf{M}_{i} \boldsymbol{\varepsilon}_{i} /(T-k-1)} \sim F(k, T-k-1)
$$

where $F\left(v_{1}, v_{2}\right)$ is a $F$ distribution with $v_{1}$ and $v_{2}$ degrees of freedom. It is well known that mean and variance of $F\left(v_{1}, v_{2}\right)$ is $v_{2} /\left(v_{2}-2\right)$ (for $\left.v_{2}>2\right)$ and

$$
\frac{2 v_{2}^{2}\left(v_{2}+v_{1}-2\right)}{v_{1}\left(v_{2}-2\right)^{2}\left(v_{2}-4\right)},\left(\text { for } v_{2}>4\right)
$$

respectively. Using these results we have

$$
\begin{gathered}
E\left[\frac{(T-k-1) \varepsilon_{i}^{\prime} \mathbf{P}_{i} \varepsilon_{i}}{\varepsilon_{i}^{\prime} \mathbf{M}_{i} \varepsilon_{i}}\right]=\frac{k(T-k-1)}{T-k-3}=k+O\left(T^{-1}\right) \\
\operatorname{Var}\left[\frac{(T-k-1) \varepsilon_{i}^{\prime} \mathbf{P}_{i} \varepsilon_{i}}{\boldsymbol{\varepsilon}_{i}^{\prime} \mathbf{M}_{i} \boldsymbol{\varepsilon}_{i}}\right]=\frac{2 k(T-k-1)^{2}(T-3)}{(T-k-3)^{2}(T-k-5)}=2 k+O\left(T^{-1}\right) .
\end{gathered}
$$

Consider now the moments of $\tilde{z}_{i T}$ under normality. Using Pitman's (1937) result, $\tilde{z}_{i T}$ and its denominator $\boldsymbol{v}_{i}^{\prime} \mathbf{M}_{\tau} \boldsymbol{v}_{i}$ are independent. Hence, $\tilde{z}_{i T}^{r}$ and $\left(\boldsymbol{v}_{i}^{\prime} \mathbf{M}_{\tau} \boldsymbol{v}_{i}\right)^{r}$ are independently distributed, 
and we have $E\left\{\tilde{z}_{i T}^{r}\left[\boldsymbol{v}_{i}^{\prime} \mathbf{M}_{\tau} \boldsymbol{v}_{i} /(T-1)\right]^{r}\right\}=E\left(\tilde{z}_{i T}^{r}\right) E\left\{\left[\boldsymbol{v}_{i}^{\prime} \mathbf{M}_{\tau} \boldsymbol{v}_{i} /(T-1)\right]^{r}\right\}$, which yields $E\left(\tilde{z}_{i T}^{r}\right)=$ $E\left[\left(\boldsymbol{v}_{i}^{\prime} \mathbf{P}_{i} \boldsymbol{v}_{i}\right)^{r}\right] / E\left\{\left[\boldsymbol{v}_{i}^{\prime} \mathbf{M}_{\tau} \boldsymbol{v}_{i} /(T-1)\right]^{r}\right\}$. Therefore

$$
E\left(\tilde{z}_{i T}\right)=\frac{E\left(\varepsilon_{i}^{\prime} \mathbf{P}_{i} \varepsilon_{i}\right)}{E\left(\varepsilon_{i}^{\prime} \mathbf{M}_{\tau} \varepsilon_{i}\right) /(T-1)}=\frac{(T-1) \operatorname{tr}\left(\mathbf{P}_{i}\right)}{\operatorname{tr}\left(\mathbf{M}_{\tau}\right)}=k,
$$

and

$$
E\left(\tilde{z}_{i T}^{2}\right)=\frac{E\left[\left(\varepsilon_{i}^{\prime} \mathbf{P}_{i} \varepsilon_{i}\right)^{2}\right]}{E\left[\left(\varepsilon_{i}^{\prime} \mathbf{M}_{\tau} \varepsilon_{i}\right)^{2}\right] /(T-1)^{2}}=\left(\frac{T-1}{T+1}\right)\left(k^{2}+2 k\right),
$$

so that

$$
\operatorname{Var}\left(\tilde{z}_{i T}\right)=\frac{2 k(T-1)-2 k^{2}}{T+1} .
$$

Using the above results in conjunction with proof of Theorem 2 now yields the desired results.

\section{A.6 Proof of Asymptotic Power}

Under the local alternatives (defined by (39))

$$
\boldsymbol{\beta}_{i}=\boldsymbol{\beta}+\frac{\boldsymbol{\delta}_{i}}{N^{1 / 4} T^{1 / 2}}
$$

we first note that

$$
\sqrt{T}\left(\hat{\boldsymbol{\beta}}_{i}-\hat{\boldsymbol{\beta}}_{W F E}\right)=\boldsymbol{\kappa}_{i N T}+\varkappa_{i N T},
$$

where

$$
\boldsymbol{\kappa}_{i N T}=\hat{\mathbf{Q}}_{i T}^{-1} \hat{\boldsymbol{\xi}}_{i T}-N^{-1 / 2} \hat{\mathbf{Q}}_{N}^{-1} \hat{\boldsymbol{\xi}}_{N}, \varkappa_{i N T}=\frac{\boldsymbol{\delta}_{i}}{N^{1 / 4}}-\frac{1}{N^{1 / 4}} \hat{\mathbf{Q}}_{N}^{-1}\left(\frac{\sum_{i=1}^{N} \hat{\mathbf{Q}}_{i T} \boldsymbol{\delta}_{i}}{N}\right),
$$

with

$$
\hat{\mathbf{Q}}_{i T}=\hat{\sigma}_{i}^{-2} \mathbf{Q}_{i T}, \hat{\boldsymbol{\xi}}_{i T}=\hat{\sigma}_{i}^{-2} \boldsymbol{\xi}_{i T},
$$

and

$$
\hat{\mathbf{Q}}_{N}=N^{-1} \sum_{i=1}^{N} \hat{\mathbf{Q}}_{i T}, \hat{\boldsymbol{\xi}}_{N}=N^{-1 / 2} \sum_{i=1}^{N} \hat{\boldsymbol{\xi}}_{i T}
$$

Hence

$$
\begin{aligned}
N^{-1} \hat{S}= & \frac{T}{N} \sum_{i=1}^{N}\left(\hat{\boldsymbol{\beta}}_{i}-\hat{\boldsymbol{\beta}}_{W F E}\right)^{\prime} \hat{\mathbf{Q}}_{i T}\left(\hat{\boldsymbol{\beta}}_{i}-\hat{\boldsymbol{\beta}}_{W F E}\right) \\
= & \frac{1}{N} \sum_{i=1}^{N} \boldsymbol{\kappa}_{i N T}^{\prime} \hat{\mathbf{Q}}_{i T} \boldsymbol{\kappa}_{i N T}+\frac{1}{N} \sum_{i=1}^{N} \varkappa_{i N T}^{\prime} \hat{\mathbf{Q}}_{i T} \varkappa_{i N T} \\
& +\frac{2}{N} \sum_{i=1}^{N} \boldsymbol{\kappa}_{i N T}^{\prime} \hat{\mathbf{Q}}_{i T} \varkappa_{i N T} .
\end{aligned}
$$

The first term is the component of the test statistic that remains under the null hypothesis and is already shown to be given by

$$
\frac{1}{N} \sum_{i=1}^{N} \boldsymbol{\kappa}_{i N T}^{\prime} \hat{\mathbf{Q}}_{i T} \boldsymbol{\kappa}_{i N T}=\frac{1}{N} \sum_{i=1}^{N} \hat{z}_{i T}+O_{p}\left(N^{-1 / 2} T^{-1 / 2}\right)+O_{p}\left(N^{-1}\right) .
$$


Similarly,

$$
\frac{1}{N} \sum_{i=1}^{N} \boldsymbol{\kappa}_{i N T}^{\prime} \hat{\mathbf{Q}}_{i T} \varkappa_{i N T}=N^{-3 / 4}\left[\left(\frac{\sum_{i=1}^{N} \hat{\boldsymbol{\xi}}_{i T}^{\prime} \boldsymbol{\delta}_{i}}{\sqrt{N}}\right)-\hat{\boldsymbol{\xi}}_{N}^{\prime} \hat{\mathbf{Q}}_{N}^{-1}\left(\frac{\sum_{i=1}^{N} \hat{\mathbf{Q}}_{i T} \boldsymbol{\delta}_{i}}{N}\right)\right],
$$

and

$$
\frac{1}{N} \sum_{i=1}^{N} \varkappa_{i N T}^{\prime} \hat{\mathbf{Q}}_{i T} \varkappa_{i N T}=\frac{\psi_{N T}}{\sqrt{N}}
$$

where

$$
\psi_{N T}=\frac{1}{N} \sum_{i=1}^{N} \boldsymbol{\delta}_{i}^{\prime} \hat{\mathbf{Q}}_{i T} \boldsymbol{\delta}_{i}-\left(\frac{1}{N} \sum_{i=1}^{N} \boldsymbol{\delta}_{i}^{\prime} \hat{\mathbf{Q}}_{i T}\right)\left(\frac{1}{N} \sum_{i=1}^{N} \hat{\mathbf{Q}}_{i T}\right)^{-1}\left(\frac{1}{N} \sum_{i=1}^{N} \hat{\mathbf{Q}}_{i T} \boldsymbol{\delta}_{i}\right) .
$$

Consider now the terms in (A.28) and first note that (as in Section A.2)

$$
\hat{\boldsymbol{\xi}}_{N}^{\prime} \hat{\mathbf{Q}}_{N}^{-1}\left(\frac{\sum_{i=1}^{N} \hat{\mathbf{Q}}_{i T} \boldsymbol{\delta}_{i}}{N}\right)=O_{p}(1) .
$$

Further

$$
\frac{\sum_{i=1}^{N} \hat{\boldsymbol{\xi}}_{i T}^{\prime} \boldsymbol{\delta}_{i}}{\sqrt{N}}=\frac{1}{\sqrt{N T}} \sum_{i=1}^{N} \frac{\boldsymbol{\varepsilon}_{i}^{\prime} \mathbf{M}_{\tau} \mathbf{X}_{i} \boldsymbol{\delta}_{i}}{(T-k-1)^{-1} \boldsymbol{\varepsilon}_{i}^{\prime} \mathbf{M}_{i} \varepsilon_{i}},
$$

and since $\varepsilon_{i}$ 's are assumed to be independently distributed, we have

$\operatorname{Var}\left(\frac{\sum_{i=1}^{N} \hat{\boldsymbol{\xi}}_{i T}^{\prime} \boldsymbol{\delta}_{i}}{\sqrt{N}}\right)=\frac{1}{N} \sum_{i=1}^{N} E\left[\frac{\left(T^{-1 / 2} \boldsymbol{\varepsilon}_{i}^{\prime} \mathbf{M}_{\tau} \mathbf{X}_{i} \boldsymbol{\delta}_{i}\right)^{2}}{\left[(T-k-1)^{-1} \boldsymbol{\varepsilon}_{i}^{\prime} \mathbf{M}_{i} \boldsymbol{\varepsilon}_{i}\right]^{2}}\right]-\frac{1}{N} \sum_{i=1}^{N}\left[E\left(\frac{T^{-1 / 2} \varepsilon_{i}^{\prime} \mathbf{M}_{\tau} \mathbf{X}_{i} \boldsymbol{\delta}_{i}}{(T-k-1)^{-1} \boldsymbol{\varepsilon}_{i}^{\prime} \mathbf{M}_{i} \boldsymbol{\varepsilon}_{i}}\right)\right]^{2}$.

But by Cauchy-Schwarz inequality and under our assumptions

$$
E\left[\frac{T^{-1 / 2} \varepsilon_{i}^{\prime} \mathbf{M}_{\tau} \mathbf{X}_{i} \boldsymbol{\delta}_{i}}{(T-k-1)^{-1} \varepsilon_{i}^{\prime} \mathbf{M}_{i} \varepsilon_{i}}\right] \leq E\left[\left(T^{-1 / 2} \varepsilon_{i}^{\prime} \mathbf{M}_{\tau} \mathbf{X}_{i} \boldsymbol{\delta}_{i}\right)^{2}\right] E\left[\frac{1}{\left[(T-k-1)^{-1} \boldsymbol{\varepsilon}_{i}^{\prime} \mathbf{M}_{i} \varepsilon_{i}\right]^{2}}\right] \leq K,
$$

and

$$
E\left[\frac{\left(T^{-1 / 2} \varepsilon_{i}^{\prime} \mathbf{M}_{\tau} \mathbf{X}_{i} \boldsymbol{\delta}_{i}\right)^{2}}{\left[(T-k-1)^{-1} \varepsilon_{i}^{\prime} \mathbf{M}_{i} \boldsymbol{\varepsilon}_{i}\right]^{2}}\right] \leq E\left[\left(T^{-1 / 2} \boldsymbol{\varepsilon}_{i}^{\prime} \mathbf{M}_{\tau} \mathbf{X}_{i} \boldsymbol{\delta}_{i}\right)^{4}\right] E\left[\frac{1}{\left[(T-k-1)^{-1} \boldsymbol{\varepsilon}_{i}^{\prime} \mathbf{M}_{i} \varepsilon_{i}\right]^{4}}\right] \leq K,
$$

and hence $\operatorname{Var}\left(N^{-1 / 2} \sum_{i=1}^{N} \hat{\boldsymbol{\xi}}_{i T}^{\prime} \boldsymbol{\delta}_{i}\right)<K$, which establishes that $N^{-1 / 2} \sum_{i=1}^{N} \hat{\boldsymbol{\xi}}_{i T}^{\prime} \boldsymbol{\delta}_{i}=O_{p}(1)$. Substituting this result together with (A.30) in (A.28) now gives $N^{-1} \sum_{i=1}^{N} \boldsymbol{\kappa}_{i N T}^{\prime} \hat{\mathbf{Q}}_{i T} \varkappa_{i N T}=$ $O_{p}\left(N^{-3 / 4}\right)$, and if used in conjunction with (A.29) and (A.27) yields

$$
N^{-1} \hat{S}=\frac{1}{N} \sum_{i=1}^{N} \hat{z}_{i T}+\frac{\psi_{N T}}{\sqrt{N}}+O_{p}\left(N^{-3 / 4}\right)+O_{p}\left(N^{-1 / 2} T^{-1 / 2}\right) .
$$

Finally, using this result in (26) gives

$$
\hat{\Delta}=\frac{1}{\sqrt{N}} \sum_{i=1}^{N}\left(\frac{\hat{z}_{i T}-k}{\sqrt{2 k}}\right)+\frac{\psi_{N T}}{\sqrt{2 k}}+O_{p}\left(N^{-1 / 4}\right)+O_{p}\left(T^{-1 / 2}\right),
$$

as required. 


\section{A.7 Proof of Theorem 3}

First note that under $H_{0}$, and Assumptions D1 to D3, $E\left|Q_{i T}\right|<K$ and $^{15}$

$$
\begin{aligned}
Q_{i T} & =\frac{\mathbf{y}_{i,-1}^{\prime} \mathbf{M}_{\tau} \mathbf{y}_{i,-1}}{T}=\frac{\sigma_{i}^{2}}{1-\beta^{2}}+O_{p}\left(T^{-1 / 2}\right) \\
Q_{N T} & =\frac{\sum_{i=1}^{N} \mathbf{y}_{i,-1}^{\prime} \mathbf{M}_{\tau} \mathbf{y}_{i,-1}}{N T}=\frac{\bar{\sigma}^{2}}{1-\beta^{2}}+O\left(T^{-1}\right)+O_{p}\left(N^{-1 / 2} T^{-1 / 2}\right),
\end{aligned}
$$

where $\bar{\sigma}^{2}=\lim _{N \rightarrow \infty} N^{-1} \sum_{i=1}^{N} \sigma_{i}^{2}$, and $0<\bar{\sigma}^{2}<K$. Also (re Alvarez and Arellano (2003, p.1140))

$$
Q_{i T}^{-1}=\frac{1-\beta^{2}}{\sigma_{i}^{2}}+O_{p}\left(T^{-1 / 2}\right)
$$

and

$$
Q_{N T}^{-1}=\frac{1-\beta^{2}}{\bar{\sigma}^{2}}+O_{p}\left(T^{-1}\right)+O_{p}\left(N^{-1 / 2} T^{-1 / 2}\right)
$$

Let

$$
\xi_{i T}=\frac{\mathbf{y}_{i,-1}^{\prime} \mathbf{M}_{\tau} \varepsilon_{i}}{\sqrt{T}}, \xi_{N T}=\frac{\sum_{i=1}^{N} \xi_{i T}}{\sqrt{N}} .
$$

Similarly (using (A10) in Alvarez and Arellano (2003))

$$
E\left(\xi_{N T}\right)=-\left(\frac{N}{T}\right)^{1 / 2} \frac{\bar{\sigma}^{2}}{1-\beta^{2}}+O\left(T^{-1} \sqrt{\frac{N}{T}}\right),
$$

and

$$
\begin{aligned}
\xi_{i T} & \rightarrow{ }_{d} N\left(0, \frac{\sigma_{i}^{2}}{1-\beta^{2}}\right) \\
\xi_{N T}-E\left(\xi_{N T}\right) & =\frac{\sum_{i=1}^{N} \mathbf{y}_{i,-1}^{\prime} \mathbf{M}_{\tau} \varepsilon_{i}}{\sqrt{N T}}-\left[-\left(\frac{N}{T}\right)^{1 / 2} \frac{\bar{\sigma}^{2}}{1-\beta^{2}}+O\left(T^{-1} \sqrt{\frac{N}{T}}\right)\right] \\
& \rightarrow d_{d} N\left(0, \frac{\bar{\sigma}^{4}}{1-\beta^{2}}\right),
\end{aligned}
$$

where $\bar{\sigma}^{4}=\lim _{N \rightarrow \infty} N^{-1} \sum_{i=1}^{N} \sigma_{i}^{4}$. Therefore, since under Assumption D2 $N / T \rightarrow \kappa$, where $0 \leq \kappa<\infty$, then $\xi_{N T}=O_{p}(1)$. It is also clear that

$$
\xi_{i T}=O_{p}(1), Q_{i T}^{-1}=O_{p}(1), \text { and } Q_{N T}^{-1}=O_{p}(1) .
$$

Using these results in

$$
\tilde{\sigma}_{i}^{2}=\frac{\boldsymbol{\varepsilon}_{i}^{\prime} \mathbf{M}_{\tau} \varepsilon_{i}}{T-1}+\frac{2 \xi_{N T} Q_{N T}^{-1} \xi_{i T}}{\sqrt{N}(T-1)}+\frac{\xi_{N T}^{2} Q_{N T}^{-2} Q_{i T}}{N(T-1)}
$$

now yields

$$
\tilde{\sigma}_{i}^{2}=\frac{\varepsilon_{i}^{\prime} \mathbf{M}_{\tau} \varepsilon_{i}}{T-1}+O_{p}\left(N^{-1 / 2} T^{-1}\right) .
$$

\footnotetext{
${ }^{15}$ See, for example, Alvarez and Arellano (2003, pp. 1127-1128 and Appendix A.1) where the results are derived under $\sigma_{i}^{2}=\sigma^{2}$.
} 
Also

$$
\tilde{\sigma}_{i}^{-2}=\sigma_{i}^{-2}+O_{p}\left(T^{-1 / 2}\right)+O_{p}\left(N^{-1 / 2} T^{-1}\right) .
$$

Assuming that $T>T_{0}$ so that $E\left(\tilde{\sigma}_{i}^{-2}\right)<K$, we now have ${ }^{16}$

$$
\begin{aligned}
& \frac{\sum_{i=1}^{N} \tilde{\sigma}_{i}^{-2} Q_{i T}}{N}=\frac{\sum_{i=1}^{N} \sigma_{i}^{-2} Q_{i T}}{N}+O_{p}\left(T^{-1 / 2}\right)+O_{p}\left(N^{-1 / 2} T^{-1}\right), \\
& \frac{\sum_{i=1}^{N} \tilde{\sigma}_{i}^{-2} \xi_{i T}}{\sqrt{N}}=\frac{\sum_{i=1}^{N} \sigma_{i}^{-2} \xi_{i T}}{\sqrt{N}}+O_{p}\left(\sqrt{\frac{N}{T}}\right)+O_{p}\left(T^{-1}\right),
\end{aligned}
$$

and under Assumptions D1 and D2 $(N / T \rightarrow \kappa, 0 \leq \kappa<\infty)$ we also have

$$
\frac{\sum_{i=1}^{N} \sigma_{i}^{-2} Q_{i T}}{N}=O_{p}(1), \frac{\sum_{i=1}^{N} \sigma_{i}^{-2} \xi_{i T}}{\sqrt{N}}=O_{p}\left(\sqrt{\frac{N}{T}}\right) .
$$

Using these results in

$$
\frac{1}{N} \tilde{S}=\frac{1}{N} \sum_{i=1}^{N} \frac{\xi_{i T}^{2} Q_{i T}^{-1}}{\tilde{\sigma}_{i}^{2}}-\frac{1}{N}\left(\frac{\sum_{i=1}^{N} \tilde{\sigma}_{i}^{-2} \xi_{i T}}{\sqrt{N}}\right)^{2}\left(\frac{\sum_{i=1}^{N} \tilde{\sigma}_{i}^{-2} Q_{i T}}{N}\right)^{-1}
$$

now gives (assuming $N / T \rightarrow \kappa, 0 \leq \kappa<\infty$ )

$$
\frac{1}{N} \tilde{S}=\frac{1}{N} \sum_{i=1}^{N} \frac{\xi_{i T}^{2} Q_{i T}^{-1}}{\tilde{\sigma}_{i}^{2}}+O_{p}\left(N^{-1}\right)+O_{p}\left(N^{-1 / 2} T^{-1 / 2}\right)
$$

Substituting for $\tilde{\sigma}_{i}^{2}=\varepsilon_{i}^{\prime} \mathbf{M}_{\tau} \varepsilon_{i} /(T-1)+O_{p}\left(N^{-1 / 2} T^{-1}\right)$, we now have

$$
\frac{1}{N} \tilde{S}=\frac{1}{N} \sum_{i=1}^{N} \frac{(T-1) \xi_{i T}^{2} Q_{i T}^{-1}}{\varepsilon_{i}^{\prime} \mathbf{M}_{\tau} \varepsilon_{i}}+O_{p}\left(N^{-1}\right)+O_{p}\left(N^{-1 / 2} T^{-1 / 2}\right),
$$

or

$$
\frac{1}{N} \tilde{S}=\frac{1}{N} \sum_{i=1}^{N} \tilde{w}_{i}+O_{p}\left(N^{-1}\right)+O_{p}\left(N^{-1 / 2} T^{-1 / 2}\right)
$$

where

$$
\tilde{w}_{i}=\frac{(T-1)\left(T^{-1 / 2} \varepsilon_{i}^{\prime} \mathbf{M}_{\tau} \mathbf{y}_{i .-1}\right)^{2}}{\left(\varepsilon_{i}^{\prime} \mathbf{M}_{\tau} \varepsilon_{i}\right)\left(T^{-1} \mathbf{y}_{i,-1}^{\prime} \mathbf{M}_{\tau} \mathbf{y}_{i,-1}\right)}
$$

\section{A.8 Proof of Theorem 4}

First note that under $H_{0}$, the $\mathrm{AR}(1)$ model, (40), can be written in matrix notations as

$$
\mathbf{y}_{i}^{*}=a_{i} \boldsymbol{\tau}_{T+1}+\mathcal{B}^{-1} \mathbf{D}_{i} \boldsymbol{\nu}_{i},
$$

where $a_{i}=(1-\beta) \alpha_{i}, \mathbf{y}_{i}^{*}=\left(y_{i 0}, y_{i 1}, \ldots, y_{i T}\right)^{\prime}, \boldsymbol{\nu}_{i}=\left(u_{i 0} / \delta_{i}, \varepsilon_{i 1} / \sigma_{i}, \ldots, \varepsilon_{i T} / \sigma_{i}\right)^{\prime}$ so that $\boldsymbol{\nu}_{i} \sim$ $N\left(\mathbf{0}_{T+1 \times 1}, \mathbf{I}_{T+1}\right), \mathbf{0}_{T+1 \times 1}$ is a $(T+1) \times 1$ vector of zeros, $\mathbf{I}_{T+1}$ is an identity matrix of order

\footnotetext{
${ }^{16}$ Note that $E\left|Q_{i T}\right|<K$ and $E\left|\xi_{i T}\right|<K$.
} 
$T+1, \boldsymbol{\tau}_{T+1}$ is a $(T+1) \times 1$ vector of ones, $\mathbf{D}_{i}$ is a $(T+1) \times(T+1)$ diagonal matrix with its first element equal to $\delta_{i}$ and the remaining elements equal to $\sigma_{i}$, and

$$
\mathcal{B}=\left[\begin{array}{ccccc}
1 & 0 & \cdots & 0 & 0 \\
-\beta & 1 & \cdots & 0 & 0 \\
\vdots & \vdots & \ddots & \vdots & \vdots \\
0 & 0 & \cdots & 1 & 0 \\
0 & 0 & \cdots & -\beta & 1
\end{array}\right]
$$

Also $\mathbf{y}_{i}=\mathbf{G}_{0} \mathbf{y}_{i}^{*}, \mathbf{y}_{i,-1}=\mathbf{G}_{1} \mathbf{y}_{i}^{*}$, where $\mathbf{G}_{0}=\left(\mathbf{0}_{T \times 1}, \mathbf{I}_{T}\right)$ and $\mathbf{G}_{1}=\left(\mathbf{I}_{T}, \mathbf{0}_{T \times 1}\right)$. Hence, noting that $\mathbf{M}_{\tau} \mathbf{G}_{1} \boldsymbol{\tau}_{T+1}=\mathbf{0}$, and using the above results in (A.33) we have

$$
\tilde{w}_{i}=\frac{(T-1)\left(T^{-1 / 2} \boldsymbol{v}_{i}^{\prime} \mathbf{M}_{\tau} \mathbf{y}_{i,-1}\right)^{2}}{\left(\boldsymbol{v}_{i}^{\prime} \mathbf{M}_{\tau} \boldsymbol{v}_{i}\right)\left(T^{-1} \mathbf{y}_{i,-1}^{\prime} \mathbf{M}_{\tau} \mathbf{y}_{i,-1}\right)}
$$

or $\tilde{w}_{i}=X_{i T} / Y_{i T}$, where $X_{i T}=\left(\boldsymbol{\nu}_{i}^{\prime} \mathbf{A}_{i} \boldsymbol{\nu}_{i}\right)^{2}, Y_{i T}=\left(\boldsymbol{\nu}_{i}^{\prime} \mathbf{B} \boldsymbol{\nu}_{i}\right)\left(\boldsymbol{\nu}_{i}^{\prime} \mathbf{C}_{i} \boldsymbol{\nu}_{i}\right)$.

$$
\begin{gathered}
\mathbf{A}_{i}=\frac{\mathbf{G}_{0}^{\prime} \mathbf{M}_{\tau} \mathbf{G}_{1} \mathcal{B}^{-1} \mathbf{D}_{i}}{\sqrt{T}}, \\
\mathbf{B}=\frac{\mathbf{G}_{0}^{\prime} \mathbf{M}_{\tau} \mathbf{G}_{0}}{T-1}
\end{gathered}
$$

and noting $\mathbf{G}_{0} \mathbf{G}_{0}^{\prime}=\mathbf{I}_{T}$,

$$
\mathbf{C}_{i}=\mathbf{A}_{i}^{\prime} \mathbf{A}_{i}=\frac{\mathbf{D}_{i} \mathcal{B}^{-1 /} \mathbf{G}_{1}^{\prime} \mathbf{M}_{\tau} \mathbf{G}_{1} \mathcal{B}^{-1} \mathbf{D}_{i}}{T}
$$

First note that $\mathcal{B}$ and $\mathbf{D}_{i}$ are non-singular matrices, and

$$
\mathbf{G}_{0}^{\prime} \mathbf{M}_{\tau} \mathbf{G}_{0}=\left(\begin{array}{cc}
\mathbf{0}_{1 \times 1} & \mathbf{0}_{1 \times T} \\
\mathbf{0}_{T \times 1} & \mathbf{M}_{\tau}
\end{array}\right), \mathbf{G}_{1}^{\prime} \mathbf{M}_{\tau} \mathbf{G}_{1}=\left(\begin{array}{cc}
\mathbf{M}_{\tau} & \mathbf{0}_{T \times 1} \\
\mathbf{0}_{1 \times T} & 0_{1 \times 1}
\end{array}\right)
$$

where $\mathbf{G}_{0}^{\prime} \mathbf{M}_{\tau} \mathbf{G}_{0}$ and $\mathbf{G}_{1}^{\prime} \mathbf{M}_{\tau} \mathbf{G}_{1}$ are idempotent matrices. Therefore, it readily follows that $\mathbf{B}$ and $\mathbf{C}_{i}$ are non-negative definite matrices with rank $T-1$. Therefore, by Corollary $2 E\left(Y_{i T}^{-4}\right)=$ $E\left[\left(\boldsymbol{\nu}_{i}^{\prime} \mathbf{B} \boldsymbol{\nu}_{i}\right)^{-4}\left(\boldsymbol{\nu}_{i}^{\prime} \mathbf{C}_{i} \boldsymbol{\nu}_{i}\right)^{-4}\right]<K$ if $T-1>16$. Also $E\left(X_{i T}^{4}\right)=E\left[\left(\boldsymbol{\nu}_{i}^{\prime} \mathbf{A}_{i} \boldsymbol{\nu}_{i}\right)^{8}\right]<K$. (see (A.54) below). Hence, if $T>17$, by Cauchy-Schwarz inequality

$$
E\left(\tilde{w}_{i}^{2}\right)=E\left(\frac{X_{i T}^{2}}{Y_{i T}^{2}}\right) \leq\left[E\left(X_{i T}^{4}\right)\right]^{1 / 2}\left[E\left(Y_{i T}^{-4}\right)\right]^{1 / 2}<K
$$

and the first two moments of $\tilde{w}_{i}$ exist for each $i$.

A mean value expansion of $\tilde{w}_{i}$ around $\varphi_{i}=E\left(Y_{i T}\right)>0$, now yields

$$
\tilde{w}_{i}=\frac{X_{i T}}{Y_{i T}}=\frac{X_{i T}}{\varphi_{i}}-\frac{X_{i T}}{\varphi_{i}^{2}}\left(Y_{i T}-\varphi_{i}\right)+\frac{X_{i T}}{\bar{Y}_{i T}^{3}}\left(Y_{i T}-\varphi_{i}\right)^{2},
$$

where $\bar{Y}_{i T}=\lambda Y_{i T}+(1-\lambda) \varphi_{i}, \lambda \in[0,1]$. But in view of (A.58) and (A.62), $\left(\boldsymbol{\nu}_{i}^{\prime} \mathbf{B} \boldsymbol{\nu}_{i}\right)\left(\boldsymbol{\nu}_{i}^{\prime} \mathbf{C}_{i} \boldsymbol{\nu}_{i}\right)=$ $O_{p}(1)$, and

$$
\frac{1}{\bar{Y}_{i T}^{3}}=\frac{1}{\left[\lambda\left(\boldsymbol{\nu}_{i}^{\prime} \mathbf{B} \boldsymbol{\nu}_{i}\right)\left(\boldsymbol{\nu}_{i}^{\prime} \mathbf{C}_{i} \boldsymbol{\nu}_{i}\right)+(1-\lambda) \varphi_{i}\right]^{3}}=O_{p}(1) .
$$


Also

$$
\begin{aligned}
E\left[X_{i T}\left(Y_{i T}-\varphi_{i}\right)^{2}\right] & \leq\left[E\left(X_{i T}^{2}\right)\right]^{1 / 2}\left[E\left(Y_{i T}-\varphi_{i}\right)^{4}\right]^{1 / 2} \\
& =O\left(T^{-1}\right)
\end{aligned}
$$

by (A.53) and (A.63). Therefore, the last term of (A.41) is $O_{p}\left(T^{-1}\right)$, and,

$$
\tilde{w}_{i}-w_{i}^{*}=O_{p}\left(T^{-1}\right)
$$

where

$$
w_{i}^{*}=\frac{\left(\boldsymbol{\nu}_{i}^{\prime} \mathbf{A}_{i} \boldsymbol{\nu}_{i}\right)^{2}}{E\left[\left(\boldsymbol{\nu}_{i}^{\prime} \mathbf{B} \boldsymbol{\nu}_{i}\right)\left(\boldsymbol{\nu}_{i}^{\prime} \mathbf{C}_{i} \boldsymbol{\nu}_{i}\right)\right]}\left[1-\frac{\left(\boldsymbol{\nu}_{i}^{\prime} \mathbf{B} \boldsymbol{\nu}_{i}\right)\left(\boldsymbol{\nu}_{i}^{\prime} \mathbf{C}_{i} \boldsymbol{\nu}_{i}\right)-E\left[\left(\boldsymbol{\nu}_{i}^{\prime} \mathbf{B} \boldsymbol{\nu}_{i}\right)\left(\boldsymbol{\nu}_{i}^{\prime} \mathbf{C}_{i} \boldsymbol{\nu}_{i}\right)\right]}{E\left[\left(\boldsymbol{\nu}_{i}^{\prime} \mathbf{B} \boldsymbol{\nu}_{i}\right)\left(\boldsymbol{\nu}_{i}^{\prime} \mathbf{C}_{i} \boldsymbol{\nu}_{i}\right)\right]}\right] .
$$

Using (A.42) in (A.32), and recalling that $E\left|\tilde{w}_{i}\right|<K$ for each $i$, we now have

$$
\frac{1}{N} \tilde{S}=\frac{1}{N} \sum_{i=1}^{N} w_{i}^{*}+O_{p}\left(T^{-1}\right)+O_{p}\left(N^{-1}\right)+O_{p}\left(N^{-1 / 2} T^{-1 / 2}\right),
$$

and

$$
\tilde{\Delta}=\frac{1}{\sqrt{N}} \sum_{i=1}^{N} \frac{\left(w_{i}^{*}-1\right)}{\sqrt{2}}+O_{p}\left(N^{1 / 2} T^{-1}\right)+O_{p}\left(N^{-1 / 2}\right)+O_{p}\left(T^{-1 / 2}\right) .
$$

Hence under $N / T \rightarrow \kappa, 0 \leq \kappa<\infty, \tilde{\Delta} \stackrel{d}{\sim} \frac{1}{\sqrt{N}} \sum_{i=1}^{N} \frac{\left(w_{i}^{*}-1\right)}{\sqrt{2}}$ as $(N, T) \stackrel{j}{\rightarrow} \infty$.

\section{A.8.1 Asymptotic Expansion of $E\left(w_{i}^{*}\right)$ and $\operatorname{Var}\left(w_{i}^{*}\right)$}

Consider $w_{i}^{*}$, defined by (A.43), and use (A.52), (A.57), and (A.58) to obtain

$$
E\left(w_{i}^{*}\right)=1+O\left(T^{-1}\right)
$$

Similarly, using (A.53) and (A.58)

$$
\frac{E\left[\left(\boldsymbol{\nu}_{i}^{\prime} \mathbf{A}_{i} \boldsymbol{\nu}_{i}\right)^{4}\right]}{\left\{E\left[\left(\boldsymbol{\nu}_{i}^{\prime} \mathbf{B} \boldsymbol{\nu}_{i}\right)\left(\boldsymbol{\nu}_{i}^{\prime} \mathbf{C}_{i} \boldsymbol{\nu}_{i}\right)\right]\right\}^{2}}=3+O\left(T^{-1}\right)
$$

Also using (A.54), (A.62) and (A.63) we have (recall that $\left.\varphi_{i}=E\left[\left(\boldsymbol{\nu}_{i}^{\prime} \mathbf{B} \boldsymbol{\nu}_{i}\right)\left(\boldsymbol{\nu}_{i}^{\prime} \mathbf{C}_{i} \boldsymbol{\nu}_{i}\right)\right]\right)$

$$
\begin{aligned}
& E\left[\left(\boldsymbol{\nu}_{i}^{\prime} \mathbf{A}_{i} \boldsymbol{\nu}_{i}\right)^{4}\left[\left(\boldsymbol{\nu}_{i}^{\prime} \mathbf{B} \boldsymbol{\nu}_{i}\right)\left(\boldsymbol{\nu}_{i}^{\prime} \mathbf{C}_{i} \boldsymbol{\nu}_{i}\right)-\varphi_{i}\right]\right] \\
\leq & \left\{E\left[\left(\boldsymbol{\nu}_{i}^{\prime} \mathbf{A}_{i} \boldsymbol{\nu}_{i}\right)^{8}\right]\right\}^{1 / 2}\left\{E\left(\left[\left(\boldsymbol{\nu}_{i}^{\prime} \mathbf{B} \boldsymbol{\nu}_{i}\right)\left(\boldsymbol{\nu}_{i}^{\prime} \mathbf{C}_{i} \boldsymbol{\nu}_{i}\right)-\varphi_{i}\right]^{2}\right)\right\}^{1 / 2} \\
= & O\left(T^{-1 / 2}\right), \\
& E\left\{\left(\boldsymbol{\nu}_{i}^{\prime} \mathbf{A}_{i} \boldsymbol{\nu}_{i}\right)^{4}\left[\left(\boldsymbol{\nu}_{i}^{\prime} \mathbf{B} \boldsymbol{\nu}_{i}\right)\left(\boldsymbol{\nu}_{i}^{\prime} \mathbf{C}_{i} \boldsymbol{\nu}_{i}\right)-\varphi_{i}\right]^{2}\right\} \\
\leq & \left\{E\left[\left(\boldsymbol{\nu}_{i}^{\prime} \mathbf{A}_{i} \boldsymbol{\nu}_{i}\right)^{8}\right]\right\}^{1 / 2}\left\{E\left(\left[\left(\boldsymbol{\nu}_{i}^{\prime} \mathbf{B} \boldsymbol{\nu}_{i}\right)\left(\boldsymbol{\nu}_{i}^{\prime} \mathbf{C}_{i} \boldsymbol{\nu}_{i}\right)-\varphi_{i}\right]^{4}\right)\right\}^{1 / 2} \\
= & O\left(T^{-1}\right) .
\end{aligned}
$$


Using (A.46), (A.47) and (A.48),

$$
E\left(w_{i}^{* 2}\right)=3+O\left(T^{-1 / 2}\right)
$$

Finally, by (A.45) and (A.49)

$$
\begin{aligned}
\operatorname{Var}\left(w_{i}^{*}\right) & =E\left(w_{i}^{* 2}\right)-\left[E\left(w_{i}^{*}\right)\right]^{2} \\
& =2+O\left(T^{-1 / 2}\right) .
\end{aligned}
$$

Using this result together with (A.45) in (A.44), and bearing in mind that $w_{i}^{*}$ are independently distributed across $i$, we have $\tilde{\Delta} \stackrel{d}{\sim} \frac{1}{\sqrt{N}} \sum_{i=1}^{N} \frac{\left(w_{i}^{*}-1\right)}{\sqrt{2}} \rightarrow_{d} N(0,1)$, as $(N, T) \stackrel{j}{\rightarrow} \infty$.

\section{A.9 Moments for Products of Quadratic Forms}

Consider the non-stochastic matrices $\mathbf{A}, \mathbf{B}$, and $\mathbf{C}$ (suppressing subscription $i$ ) defined in Appendix A.8 by (A.37), (A.38), and (A.39), respectively, suppose that $\boldsymbol{\nu} \sim \operatorname{IIDN}\left(\mathbf{0}, \mathbf{I}_{T+1}\right)$, and let

$$
\operatorname{tr}(\mathbf{A})=a, \operatorname{tr}(\mathbf{B})=b=1, \operatorname{tr}(\mathbf{C})=c .
$$

Then

$$
\begin{aligned}
E\left[\left(\boldsymbol{\nu}^{\prime} \mathbf{A} \boldsymbol{\nu}\right)^{2}\right]=[\operatorname{tr}(\mathbf{A})]^{2}+\operatorname{tr}\left(\mathbf{A}^{2}+\mathbf{A}^{\prime} \mathbf{A}\right) & =c+O\left(T^{-1}\right) \\
E\left[\left(\boldsymbol{\nu}^{\prime} \mathbf{A} \boldsymbol{\nu}\right)^{4}\right]= & {[\operatorname{tr}(\mathbf{A})]^{4}+4 \operatorname{tr}(\mathbf{A})\left[2 \operatorname{tr}\left(\mathbf{A}^{3}\right)+6 \operatorname{tr}\left(\mathbf{A}^{\prime} \mathbf{A}^{2}\right)\right] } \\
& +3\left[\operatorname{tr}\left(\mathbf{A}^{\prime} \mathbf{A}\right)+\operatorname{tr}\left(\mathbf{A}^{2}\right)\right]^{2}+6[\operatorname{tr}(\mathbf{A})]^{2}\left[\operatorname{tr}\left(\mathbf{A}^{\prime} \mathbf{A}\right)+\operatorname{tr}\left(\mathbf{A}^{2}\right)\right] \\
+ & 6\left\{\operatorname{tr}\left(\mathbf{A}^{4}\right)+3 \operatorname{tr}\left(\mathbf{A}^{3} \mathbf{A}^{\prime}\right)+2 \operatorname{tr}\left[\left(\mathbf{A}^{\prime} \mathbf{A}\right)^{2}\right]+2 \operatorname{tr}\left[\left(\mathbf{A}^{\prime}\right)^{2} \mathbf{A}^{2}\right]\right\} \\
= & 3 c^{2}+O\left(T^{-1}\right), \\
& E\left[\left(\boldsymbol{\nu}^{\prime} \mathbf{A} \boldsymbol{\nu}\right)^{8}\right]=105 c^{4}+O\left(T^{-1}\right), \\
E\left[\left(\boldsymbol{\nu}^{\prime} \mathbf{A} \boldsymbol{\nu}\right)^{2}\left(\boldsymbol{\nu}^{\prime} \mathbf{C} \boldsymbol{\nu}\right)\right]= & {[\operatorname{tr}(\mathbf{A})]^{2} \operatorname{tr}(\mathbf{C})+4 \operatorname{tr}\left(\mathbf{A}^{2} \mathbf{C}\right)+2 \operatorname{tr}\left(\mathbf{A}^{\prime} \mathbf{A} \mathbf{C}\right)+2 \operatorname{tr}\left(\mathbf{A A}^{\prime} \mathbf{C}\right) } \\
& +4 \operatorname{tr}(\mathbf{A}) \operatorname{tr}(\mathbf{A C})+\operatorname{tr}(\mathbf{C}) \operatorname{tr}\left(\mathbf{A}^{2}+\mathbf{A}^{\prime} \mathbf{A}\right) \\
= & \operatorname{tr}(\mathbf{C}) \operatorname{tr}\left(\mathbf{A}^{\prime} \mathbf{A}\right)+O\left(T^{-1}\right) \\
= & c^{2}+O\left(T^{-1}\right), \\
& \\
= & {[\operatorname{tr}(\mathbf{A})]^{2} \operatorname{tr}(\mathbf{B})+4 \operatorname{tr}\left(\mathbf{A}^{2} \mathbf{B}\right)+2 \operatorname{tr}\left(\mathbf{A}^{\prime} \mathbf{A B}\right)+2 \operatorname{tr}\left(\mathbf{A A}^{\prime} \mathbf{B}\right) } \\
& +4 \operatorname{tr}(\mathbf{A}) \operatorname{tr}(\mathbf{A B})+\operatorname{tr}(\mathbf{B}) \operatorname{tr}\left(\mathbf{A}^{2}+\mathbf{A}^{\prime} \mathbf{A}\right) \\
= & \operatorname{tr}(\mathbf{B}) \operatorname{tr}\left(\mathbf{A}^{\prime} \mathbf{A}\right)+O\left(T^{-1}\right) \\
= & c+O\left(T^{-1}\right),
\end{aligned}
$$




$$
\begin{aligned}
& E\left[\left(\boldsymbol{\nu}^{\prime} \mathbf{A} \boldsymbol{\nu}\right)^{2}\left(\boldsymbol{\nu}^{\prime} \mathbf{B} \boldsymbol{\nu}\right)\left(\boldsymbol{\nu}^{\prime} \mathbf{C} \boldsymbol{\nu}\right)\right]=[\operatorname{tr}(\mathbf{A})]^{2} \operatorname{tr}(\mathbf{B}) \operatorname{tr}(\mathbf{C}) \\
& +8 \operatorname{tr}(\mathbf{A}) \operatorname{tr}\left(\mathbf{A B C}+\mathbf{A}^{\prime} \mathbf{B C}\right)+\operatorname{tr}(\mathbf{B})\left[4 \operatorname{tr}\left(\mathbf{A}^{2} \mathbf{C}\right)+2 \operatorname{tr}\left(\mathbf{A}^{\prime} \mathbf{A C}\right)+2 \operatorname{tr}\left(\mathbf{A A}^{\prime} \mathbf{C}\right)\right] \\
& +\operatorname{tr}(\mathbf{C})\left[4 \operatorname{tr}\left(\mathbf{A}^{2} \mathbf{B}\right)+2 \operatorname{tr}\left(\mathbf{A}^{\prime} \mathbf{A B}\right)+2 \operatorname{tr}\left(\mathbf{A} \mathbf{A}^{\prime} \mathbf{B}\right)\right] \\
& +2 \operatorname{tr}\left(\mathbf{A}^{2}\right) \operatorname{tr}(\mathbf{B C})+2 \operatorname{tr}\left(\mathbf{A}^{\prime} \mathbf{A}\right) \operatorname{tr}(\mathbf{B C})+8 \operatorname{tr}(\mathbf{A B}) \operatorname{tr}(\mathbf{A C}) \\
& +2[\operatorname{tr}(\mathbf{A})]^{2} \operatorname{tr}(\mathbf{B C})+4 \operatorname{tr}(\mathbf{A}) \operatorname{tr}(\mathbf{B}) \operatorname{tr}(\mathbf{A C})+4 \operatorname{tr}(\mathbf{A}) \operatorname{tr}(\mathbf{C}) \operatorname{tr}(\mathbf{A B}) \\
& +\operatorname{tr}(\mathbf{B}) \operatorname{tr}(\mathbf{C}) \operatorname{tr}\left(\mathbf{A}^{2}\right)+\operatorname{tr}(\mathbf{B}) \operatorname{tr}(\mathbf{C}) \operatorname{tr}\left(\mathbf{A}^{\prime} \mathbf{A}\right) \\
& +8 \operatorname{tr}\left(\mathbf{A}^{2} \mathbf{B C}\right)+8 \operatorname{tr}\left(\mathbf{A}^{\prime} \mathbf{A B C}\right)+8 \operatorname{tr}\left(\mathbf{A A}^{\prime} \mathbf{B C}\right)+8 \operatorname{tr}\left(\mathbf{A}^{2} \mathbf{C B}\right) \\
& +8 t r(\mathbf{A B A C})+4 t r\left(\mathbf{A}^{\prime} \mathbf{B} \mathbf{A C}\right)+4 t r\left(\mathbf{A B A}^{\prime} \mathbf{C}\right) \\
& =\operatorname{tr}(\mathbf{B}) \operatorname{tr}(\mathbf{C}) \operatorname{tr}\left(\mathbf{A}^{\prime} \mathbf{A}\right)+O\left(T^{-1}\right) \\
& =c^{2}+O\left(T^{-1}\right) \\
& E\left[\left(\boldsymbol{\nu}^{\prime} \mathbf{B} \boldsymbol{\nu}\right)\left(\boldsymbol{\nu}^{\prime} \mathbf{C} \boldsymbol{\nu}\right)\right]=\operatorname{tr}(\mathbf{B}) \operatorname{tr}(\mathbf{C})+2 \operatorname{tr}(\mathbf{C B})=c+O\left(T^{-1}\right) \\
& E\left[\left(\boldsymbol{\nu}^{\prime} \mathbf{B} \boldsymbol{\nu}\right)^{2}\left(\boldsymbol{\nu}^{\prime} \mathbf{C} \boldsymbol{\nu}\right)^{2}\right]=[\operatorname{tr}(\mathbf{B})]^{2}[\operatorname{tr}(\mathbf{C})]^{2}+16\left\{\operatorname{tr}(\mathbf{C}) \operatorname{tr}\left(\mathbf{C B}^{2}\right)+\operatorname{tr}(\mathbf{B}) \operatorname{tr}\left(\mathbf{B C}^{2}\right)\right\} \\
& +4\left\{\operatorname{tr}\left(\mathbf{C}^{2}\right) \operatorname{tr}\left(\mathbf{B}^{2}\right)+2[\operatorname{tr}(\mathbf{C B})]^{2}\right\}+2\left\{[\operatorname{tr}(\mathbf{C})]^{2} \operatorname{tr}\left(\mathbf{B}^{2}\right)+4 \operatorname{tr}(\mathbf{B}) \operatorname{tr}(\mathbf{C}) \operatorname{tr}(\mathbf{B C})+[\operatorname{tr}(\mathbf{B})]^{2} \operatorname{tr}\left(\mathbf{C}^{2}\right)\right\} \\
& +16\left\{\operatorname{tr}\left[(\mathbf{C B})^{2}\right]+2 \operatorname{tr}\left(\mathbf{C}^{2} \mathbf{B}^{2}\right)\right\} \\
& =c^{2}+O\left(T^{-1}\right) \\
& E\left[\left(\boldsymbol{\nu}^{\prime} \mathbf{B} \boldsymbol{\nu}\right)^{3}\left(\boldsymbol{\nu}^{\prime} \mathbf{C} \boldsymbol{\nu}\right)^{3}\right]=[\operatorname{tr}(\mathbf{B})]^{3}[\operatorname{tr}(\mathbf{C})]^{3}+6[\operatorname{tr}(\mathbf{B})]^{3} \operatorname{tr}(\mathbf{C}) \operatorname{tr}\left(\mathbf{C}^{2}\right) \\
& +18[\operatorname{tr}(\mathbf{B})]^{2}[\operatorname{tr}(\mathbf{C})]^{2} \operatorname{tr}(\mathbf{B C})+6 \operatorname{tr}(\mathbf{B})[\operatorname{tr}(\mathbf{C})]^{3} \operatorname{tr}\left(\mathbf{B}^{2}\right)+O\left(T^{-2}\right), \\
& E\left[\left(\boldsymbol{\nu}^{\prime} \mathbf{B} \boldsymbol{\nu}\right)^{4}\left(\boldsymbol{\nu}^{\prime} \mathbf{C} \boldsymbol{\nu}\right)^{4}\right]=[\operatorname{tr}(\mathbf{B})]^{4}[\operatorname{tr}(\mathbf{C})]^{4} \\
& +12[\operatorname{tr}(\mathbf{B})]^{4}[\operatorname{tr}(\mathbf{C})]^{2}\left[\operatorname{tr}\left(\mathbf{C}^{2}\right)\right]+32[\operatorname{tr}(\mathbf{B})]^{3}[\operatorname{tr}(\mathbf{C})]^{3} \operatorname{tr}(\mathbf{B C})+12[\operatorname{tr}(\mathbf{B})]^{2}[\operatorname{tr}(\mathbf{C})]^{4} \operatorname{tr}\left(\mathbf{B}^{2}\right) \\
& +32[\operatorname{tr}(\mathbf{B})]^{4} \operatorname{tr}(\mathbf{C}) \operatorname{tr}\left(\mathbf{C}^{3}\right)+O\left(T^{-2}\right) \text {. }
\end{aligned}
$$

By using (A.58) to (A.61), it is easily shown that

$$
\begin{array}{r}
\operatorname{Var}\left[\left(\boldsymbol{\nu}^{\prime} \mathbf{B} \boldsymbol{\nu}\right)\left(\boldsymbol{\nu}^{\prime} \mathbf{C} \boldsymbol{\nu}\right)\right]=O\left(T^{-1}\right), \\
E\left\{\left(\boldsymbol{\nu}^{\prime} \mathbf{B} \boldsymbol{\nu}\right)\left(\boldsymbol{\nu}^{\prime} \mathbf{C} \boldsymbol{\nu}\right)-E\left[\left(\boldsymbol{\nu}^{\prime} \mathbf{B} \boldsymbol{\nu}\right)\left(\boldsymbol{\nu}^{\prime} \mathbf{C} \boldsymbol{\nu}\right)\right]\right\}^{4}=O\left(T^{-2}\right) .
\end{array}
$$

\section{A.10 Results on Trace of Matrices}

Consider the non-stochastic matrices $\mathbf{A}, \mathbf{B}$, and $\mathbf{C}$ defined by (A.37), (A.38), and (A.39) in Appendix A.8, respectively (suppressing the subscript $i$ ). Then,

$$
\begin{aligned}
\operatorname{tr}(\mathbf{B}) & =1, \operatorname{tr}\left(\mathbf{A}^{\prime} \mathbf{A}\right)=\operatorname{tr}(\mathbf{C})=O(1), \operatorname{tr}\left(\mathbf{B}^{s}\right)=O\left(T^{-(s-1)}\right) \\
\operatorname{tr}\left(\mathbf{C}^{s}\right) & =O\left(T^{-(s-1)}\right), \operatorname{tr}(\mathbf{A})=O\left(T^{-1 / 2}\right), \operatorname{tr}\left(\mathbf{A}^{2}\right)=O\left(T^{-1}\right)
\end{aligned}
$$




$$
\begin{gathered}
\operatorname{tr}\left(\mathbf{A}^{\prime} \mathbf{C}\right)=O\left(T^{-1 / 2}\right), \operatorname{tr}\left(\mathbf{A}^{\prime} \mathbf{A} \mathbf{C}\right)=O\left(T^{-1}\right), \operatorname{tr}\left(\mathbf{A} \mathbf{A}^{\prime} \mathbf{C}\right)=O\left(T^{-1}\right), \operatorname{tr}\left(\mathbf{A}^{2} \mathbf{C}\right)=O\left(T^{-1}\right) \\
\operatorname{tr}\left(\mathbf{A}^{\prime} \mathbf{A B}\right)=O\left(T^{-1}\right), \operatorname{tr}\left(\mathbf{A} \mathbf{A}^{\prime} \mathbf{B}\right)=O\left(T^{-1}\right), \operatorname{tr}(\mathbf{A B})=O\left(T^{-3 / 2}\right), \operatorname{tr}\left(\mathbf{A}^{2} \mathbf{B}\right)=O\left(T^{-1}\right) \\
\operatorname{tr}(\mathbf{B C})=O\left(T^{-1}\right), \operatorname{tr}\left(\mathbf{A}^{\prime} \mathbf{B C}\right)=O\left(T^{-3 / 2}\right), \operatorname{tr}(\mathbf{A B C})=O\left(T^{-3 / 2}\right),
\end{gathered}
$$

and

$$
\begin{aligned}
& \operatorname{tr}\left(\mathbf{A}^{2} \mathbf{B C}\right), \operatorname{tr}\left(\mathbf{A}^{\prime} \mathbf{A B C}\right), \operatorname{tr}\left(\mathbf{A A}^{\prime} \mathbf{B C}\right), \operatorname{tr}\left(\mathbf{A}^{2} \mathbf{C B}\right), \\
& \operatorname{tr}(\mathbf{A B A C}), \operatorname{tr}\left(\mathbf{A}^{\prime} \mathbf{B} \mathbf{A C}\right), \operatorname{tr}\left(\mathbf{A B} \mathbf{A}^{\prime} \mathbf{C}\right) \text { are at most } O\left(T^{-2}\right) .
\end{aligned}
$$

Proof. We first note that

$$
\mathbf{H}_{01}=\mathbf{G}_{0}^{\prime} \mathbf{M}_{\tau} \mathbf{G}_{1}=\left(\begin{array}{cc}
\mathbf{0}_{1 \times T} & 0_{1 \times 1} \\
\mathbf{M}_{\tau} & \mathbf{0}_{T \times 1}
\end{array}\right)
$$

and $\mathbf{G}_{0}^{\prime} \mathbf{M}_{\tau} \mathbf{G}_{0}$ and $\mathbf{G}_{1}^{\prime} \mathbf{M}_{\tau} \mathbf{G}_{1}$, defined by (A.40), are $(T+1) \times(T+1)$ idempotent matrices with two zero eigenvalues and $T-1$ unit eigenvalues. Also since $\mathcal{B}$, defined by (A.35), is a lower triangular matrix with unit diagonal elements and $\mathbf{D}$ is a diagonal matrix with $\sigma_{\max }=\operatorname{Max}(\sigma, \delta)<K$ we have, using (A.39),

$$
0 \leq \nu_{t}(\mathbf{C}) \leq \frac{\sigma_{\max }}{T}
$$

where $\nu_{t}(\mathbf{C})$ for $t=0,1, \ldots, T$ are the eigenvalues of $\mathbf{C}$. Also it is easily verified that

$$
\begin{gathered}
\mathbf{A}^{\prime} \mathbf{B}=\frac{\mathcal{B}^{\prime-1} \mathbf{G}_{1}^{\prime} \mathbf{M}_{\tau} \mathbf{G}_{0} \mathbf{G}_{0}^{\prime} \mathbf{M}_{\tau} \mathbf{G}_{0}}{T^{1 / 2}(T-1)}=(T-1)^{-1} \mathbf{A}^{\prime} \\
\mathbf{A} \mathbf{A}^{\prime} \mathbf{B}=(T-1)^{-1} \mathbf{A} \mathbf{A}^{\prime}
\end{gathered}
$$

To prove the results in (A.64), we first note that

$$
\begin{gathered}
\operatorname{tr}(\mathbf{B})=1, \operatorname{tr}\left(\mathbf{B}^{s}\right)=\frac{\operatorname{tr}\left[\left(\mathbf{G}_{0}^{\prime} \mathbf{M}_{\tau} \mathbf{G}_{0}\right)\right]}{(T-1)^{s}}=\frac{1}{(T-1)^{s-1}}=O\left(T^{-(s-1)}\right), \\
\operatorname{tr}(\mathbf{C})=\sum_{t=0}^{T} \nu_{t}(\mathbf{C}) \leq \frac{(T+1) \sigma_{\max }}{T}=O(1),
\end{gathered}
$$

thus

$$
\operatorname{tr}\left(\mathbf{C}^{s}\right)=\sum_{t=0}^{T} \nu_{t}^{s}(\mathbf{C}) \leq \frac{(T+1) \sigma_{\max }}{T^{s}}=O\left(T^{-(s-1)}\right) .
$$

Since $\sigma_{\max }$ is bounded, to simplify the derivations and without loss of generality in what follows we set $\delta=\sigma=1$, (so that $\left.\mathbf{D}=\mathbf{I}_{T+1}\right)$ and note that

$$
\mathcal{B}^{-1}=\left[\begin{array}{ccccc}
1 & 0 & \cdots & 0 & 0 \\
\beta & 1 & \cdots & 0 & 0 \\
\vdots & \vdots & \ddots & \vdots & \vdots \\
\beta^{T-1} & \beta^{T-2} & \cdots & 1 & 0 \\
\beta^{T} & \beta^{T-1} & \cdots & \beta & 1
\end{array}\right]
$$




$$
\begin{gathered}
\mathbf{A}=T^{-1 / 2} \mathbf{G}_{0}^{\prime} \mathbf{M}_{\tau} \mathbf{G}_{1} \mathcal{B}^{-1} \\
= \\
\left.\mathbf{E}=\left[\begin{array}{cccccc}
0 & 0 & \cdots & 0 & 0 & 0 \\
1 & 0 & \cdots & 0 & 0 & 0 \\
\beta & 1 & \cdots & 0 & 0 & 0 \\
\vdots & \vdots & \ddots & \vdots & \vdots & \vdots \\
\beta^{T-2} & \beta^{T-3} & \cdots & 1 & 0 & 0 \\
\beta^{T-1} & \beta^{T-2} & \cdots & \beta & 1 & 0
\end{array}\right], \mathbf{E}-\mathbf{F}\right), \\
\end{gathered}
$$

where

$$
g_{\ell}=\frac{1}{T} \sum_{j=0}^{\ell} \beta^{j}=\frac{1}{T}\left(\frac{1-\beta^{\ell+1}}{1-\beta}\right)=O\left(T^{-1}\right) \quad(\text { since }|\beta|<1), \text { for } \ell=0,1, \ldots, T-1 .
$$

Therefore,

$$
\operatorname{tr}(\mathbf{A})=\frac{-1}{\sqrt{T}} \sum_{\ell=0}^{T-2} g_{\ell}=\frac{-1}{T \sqrt{T}} \sum_{\ell=0}^{T-2}\left(\frac{1-\beta^{\ell+1}}{1-\beta}\right)=O\left(T^{-1 / 2}\right) .
$$

Consider now $\operatorname{tr}\left(\mathbf{A}^{2}\right)$. Using (A.72)

$$
\operatorname{tr}\left(\mathbf{A}^{2}\right)=T^{-1}\left[\operatorname{tr}\left(\mathbf{E}^{2}\right)+\operatorname{tr}\left(\mathbf{F}^{2}\right)-2 \operatorname{tr}(\mathbf{E F})\right] .
$$

But it is easily seen that

$$
\begin{aligned}
& \operatorname{tr}\left(\mathbf{E}^{2}\right)=0 \\
& \operatorname{tr}\left(\mathbf{F}^{2}\right)=\left(\sum_{\ell=0}^{T-1} g_{\ell}\right)\left(\sum_{\ell=0}^{T-2} g_{\ell}\right)=O(1) \\
& \operatorname{tr}(\mathbf{E F})=\sum_{\ell=0}^{T-3}\left(\frac{1-\beta^{T-\ell-2}}{1-\beta}\right) g_{\ell}=\frac{1}{T} \sum_{\ell=0}^{T-3}\left(\frac{1-\beta^{T-\ell-2}}{1-\beta}\right)\left(\frac{1-\beta^{\ell+1}}{1-\beta}\right)=O(1),
\end{aligned}
$$

which together with (A.74) establishes that $\operatorname{tr}\left(\mathbf{A}^{2}\right)=O\left(T^{-1}\right)$.

To prove the results in (A.65), we observe that ${ }^{17}$

$$
\operatorname{tr}\left(\mathbf{A}^{\prime} \mathbf{A C}\right)=\operatorname{tr}\left(\mathbf{C}^{2}\right)=\sum_{t=0}^{T} \nu_{t}^{2}(\mathbf{C}) \leq \frac{\sigma_{\max }}{T}=O\left(T^{-1}\right) .
$$

By Cauchy-Schwarz inequality

$$
\left[\operatorname{tr}\left(\mathbf{A} \mathbf{A}^{\prime} \mathbf{C}\right)\right]^{2} \leq \operatorname{tr}\left(\mathbf{A A}^{\prime} \mathbf{A} \mathbf{A}^{\prime}\right) \operatorname{tr}\left(\mathbf{C}^{\prime} \mathbf{C}\right)=\operatorname{tr}\left(\left[\mathbf{A}^{\prime} \mathbf{A}\right]^{2}\right) \operatorname{tr}\left(\mathbf{C}^{2}\right)=\left[\operatorname{tr}\left(\mathbf{C}^{2}\right)\right]^{2},
$$

which establishes $\left|\operatorname{tr}\left(\mathbf{A A}^{\prime} \mathbf{C}\right)\right|=O\left(T^{-1}\right)$. Similarly, again by Cauchy-Schwarz inequality and noting that $\mathbf{A}^{\prime} \mathbf{A}=\mathbf{C}$,

$$
\left[\operatorname{tr}\left(\mathbf{A}^{2} \mathbf{C}\right)\right]^{2} \leq \operatorname{tr}\left(\mathbf{A} \mathbf{A} \mathbf{A}^{\prime} \mathbf{A}^{\prime}\right) \operatorname{tr}\left(\mathbf{C}^{2}\right)=\operatorname{tr}\left(\mathbf{A A}^{\prime} \mathbf{C}\right) \operatorname{tr}\left(\mathbf{C}^{2}\right)
$$

\footnotetext{
${ }^{17}$ Recall that $\mathbf{C}^{\prime}=\mathbf{C}$ and $\mathbf{A}^{\prime} \mathbf{A}=\mathbf{C}$.
} 
which establishes $\left|\operatorname{tr}\left(\mathbf{A}^{2} \mathbf{C}\right)\right|=O\left(T^{-1}\right)$. To derive the order of $\operatorname{tr}\left(\mathbf{A}^{\prime} \mathbf{C}\right)$, again by Cauchy-Schwarz inequality

$$
\left[\operatorname{tr}\left(\mathbf{A}^{\prime} \mathbf{C}\right)\right]^{2} \leq \operatorname{tr}\left(\mathbf{A}^{\prime} \mathbf{A}\right) \operatorname{tr}\left(\mathbf{C}^{\prime} \mathbf{C}\right)=\operatorname{tr}(\mathbf{C}) \operatorname{tr}\left(\mathbf{C}^{2}\right) .
$$

Therefore, since $\operatorname{tr}(\mathbf{C})=O(1)$, it follows that $\left|\operatorname{tr}\left(\mathbf{A}^{\prime} \mathbf{C}\right)\right|=O\left(T^{-1 / 2}\right)$.

To establish the results in (A.66), by Cauchy-Schwarz inequality

$$
\left[\operatorname{tr}\left(\mathbf{A}^{2} \mathbf{B}\right)\right]^{2} \leq \operatorname{tr}\left(\mathbf{A A}^{\prime} \mathbf{C}\right) \operatorname{tr}\left(\mathbf{B}^{2}\right)
$$

But

$$
\operatorname{tr}\left(\mathbf{B}^{2}\right)=\frac{\operatorname{tr}\left[\left(\mathbf{G}_{0}^{\prime} \mathbf{M}_{\tau} \mathbf{G}_{0}\right)^{2}\right]}{(T-1)^{2}}=\frac{\operatorname{tr}\left[\left(\mathbf{G}_{0}^{\prime} \mathbf{M}_{\tau} \mathbf{G}_{0}\right)\right]}{(T-1)^{2}}=\frac{1}{T-1}=O\left(T^{-1}\right),
$$

hence, $\left|\operatorname{tr}\left(\mathbf{A}^{2} \mathbf{B}\right)\right|=O\left(T^{-1}\right)$. Similarly,

$$
\left[\operatorname{tr}\left(\mathbf{A}^{\prime} \mathbf{A B}\right)\right]^{2}=[\operatorname{tr}(\mathbf{C B})]^{2} \leq \operatorname{tr}\left(\mathbf{C}^{2}\right) \operatorname{tr}\left(\mathbf{B}^{2}\right)=O\left(T^{-2}\right)
$$

which establishes $\left|\operatorname{tr}\left(\mathbf{A}^{\prime} \mathbf{A B}\right)\right|=O\left(T^{-1}\right)$. Using (A.70)

$$
\operatorname{tr}\left(\mathbf{A A}^{\prime} \mathbf{B}\right)=(T-1)^{-1} \operatorname{tr}\left(\mathbf{A}^{\prime} \mathbf{A}\right)=(T-1)^{-1} \operatorname{tr}(\mathbf{C})=O\left(T^{-1}\right) .
$$

Also

$$
\begin{aligned}
\operatorname{tr}(\mathbf{A B}) & =T^{-1 / 2}(T-1)^{-1} \operatorname{tr}\left(\mathbf{G}_{0}^{\prime} \mathbf{M}_{\tau} \mathbf{G}_{1} \mathcal{B}^{\prime-1} \mathbf{G}_{0}^{\prime} \mathbf{M}_{\tau} \mathbf{G}_{0}\right) \\
& =T^{-1 / 2}(T-1)^{-1} \operatorname{tr}\left(\mathbf{G}_{0}^{\prime} \mathbf{M}_{\tau} \mathbf{G}_{1} \mathcal{B}^{\prime-1}\right)=\frac{1}{T-1} \operatorname{tr}(\mathbf{A})=O\left(T^{-3 / 2}\right) .
\end{aligned}
$$

To prove the results in (A.67), a further application of the Cauchy-Schwarz inequality to $\mathbf{A}$ and BC now yields

$$
\begin{aligned}
{\left[\operatorname{tr}\left(\mathbf{A}^{\prime} \mathbf{B C}\right)\right]^{2} } & \leq \operatorname{tr}\left(\mathbf{A}^{\prime} \mathbf{A}\right) \operatorname{tr}\left(\mathbf{C}^{\prime} \mathbf{B}^{\prime} \mathbf{B C}\right)=\operatorname{tr}(\mathbf{C}) \operatorname{tr}\left(\mathbf{B}^{2} \mathbf{C}^{2}\right) \\
{[\operatorname{tr}(\mathbf{A B C})]^{2} } & \leq \operatorname{tr}\left(\mathbf{A} \mathbf{A}^{\prime}\right) \operatorname{tr}\left(\mathbf{C}^{\prime} \mathbf{B}^{\prime} \mathbf{B C}\right)=\operatorname{tr}(\mathbf{C}) \operatorname{tr}\left(\mathbf{B}^{2} \mathbf{C}^{2}\right) .
\end{aligned}
$$

But as easily seen

$$
\left[\operatorname{tr}\left(\mathbf{B}^{2} \mathbf{C}^{2}\right)\right]^{2} \leq \operatorname{tr}\left(\mathbf{B}^{4}\right) \operatorname{tr}\left(\mathbf{C}^{4}\right) \leq O\left(T^{-6}\right)
$$

so that

$$
\left|\operatorname{tr}\left(\mathbf{B}^{2} \mathbf{C}^{2}\right)\right| \leq O\left(T^{-3}\right)
$$

and hence

$$
\left|\operatorname{tr}\left(\mathbf{A}^{\prime} \mathbf{B C}\right)\right|=O\left(T^{-3 / 2}\right) \text {, and }|\operatorname{tr}(\mathbf{A B C})|=O\left(T^{-3 / 2}\right) .
$$

Similarly,

$$
[\operatorname{tr}(\mathbf{B C})]^{2} \leq \operatorname{tr}\left(\mathbf{B}^{2}\right) \operatorname{tr}\left(\mathbf{C}^{2}\right)=O\left(T^{-2}\right)
$$

and $|\operatorname{tr}(\mathbf{B C})|=O\left(T^{-1}\right)$.

Finally, the various higher order terms in (A.68) can be established following similar lines. Firstly,

$$
\operatorname{tr}\left(\mathbf{A}^{\prime} \mathbf{A B C}\right)=\operatorname{tr}\left(\mathbf{B C}^{2}\right),\left[\operatorname{tr}\left(\mathbf{B C}^{2}\right)\right]^{2} \leq \operatorname{tr}\left(\mathbf{B}^{2}\right) \operatorname{tr}\left(\mathbf{C}^{4}\right)=O\left(T^{-4}\right),
$$


so that $\left|\operatorname{tr}\left(\mathbf{B C}^{2}\right)\right|=O\left(T^{-2}\right)$, and

$$
\begin{aligned}
& {\left[\operatorname{tr}\left(\mathbf{A}^{2} \mathbf{B C}\right)\right]^{2} \leq \operatorname{tr}\left(\mathbf{A A}^{\prime} \mathbf{C}\right) \operatorname{tr}\left(\mathbf{C}^{2} \mathbf{B}^{2}\right)=O\left(T^{-4}\right)} \\
& {\left[\operatorname{tr}\left(\mathbf{A}^{2} \mathbf{C B}\right)\right]^{2} \leq \operatorname{tr}\left(\mathbf{A A}^{\prime} \mathbf{C}\right) \operatorname{tr}\left(\mathbf{C}^{2} \mathbf{B}^{2}\right)=O\left(T^{-4}\right)}
\end{aligned}
$$

Similarly,

$$
[\operatorname{tr}(\mathbf{A B A C})]^{2} \leq \operatorname{tr}\left(\mathbf{A B B}^{\prime} \mathbf{A}^{\prime}\right) \operatorname{tr}\left(\mathbf{C}^{\prime} \mathbf{A}^{\prime} \mathbf{A C}\right)=\operatorname{tr}\left(\mathbf{B}^{2} \mathbf{C}\right) \operatorname{tr}\left(\mathbf{C}^{3}\right)=O\left(T^{-4}\right) .
$$

Furthermore,

$$
\left[\operatorname{tr}\left(\mathbf{A A}^{\prime} \mathbf{B C}\right)\right]^{2}=\left[\operatorname{tr}\left(\mathbf{A}^{\prime} \mathbf{B C A}\right)\right]^{2} \leq \operatorname{tr}\left(\mathbf{A}^{\prime} \mathbf{B B}^{\prime} \mathbf{A}\right) \operatorname{tr}\left(\mathbf{A}^{\prime} \mathbf{C}^{\prime} \mathbf{C A}\right)=\operatorname{tr}\left(\mathbf{B}^{2} \mathbf{A} \mathbf{A}^{\prime}\right) \operatorname{tr}\left(\mathbf{C}^{2} \mathbf{A} \mathbf{A}^{\prime}\right)
$$

and

$$
\begin{aligned}
{\left[t\left(\mathbf{A}^{\prime} \mathbf{B} \mathbf{A C}\right)\right]^{2} } & \leq \operatorname{tr}\left(\mathbf{A}^{\prime} \mathbf{B} \mathbf{B}^{\prime} \mathbf{A}\right) \operatorname{tr}\left(\mathbf{C}^{\prime} \mathbf{A}^{\prime} \mathbf{A C}\right)=\operatorname{tr}\left(\mathbf{B}^{2} \mathbf{A} \mathbf{A}^{\prime}\right) \operatorname{tr}\left(\mathbf{C}^{3}\right) \\
{\left[\operatorname{tr}\left(\mathbf{A} \mathbf{B} \mathbf{A}^{\prime} \mathbf{C}\right)\right]^{2} } & \leq \operatorname{tr}\left(\mathbf{A B B}^{\prime} \mathbf{A}^{\prime}\right) \operatorname{tr}\left(\mathbf{C}^{\prime} \mathbf{A} \mathbf{A}^{\prime} \mathbf{C}\right)=\operatorname{tr}\left(\mathbf{B}^{2} \mathbf{C}\right) \operatorname{tr}\left(\mathbf{C}^{2} \mathbf{A} \mathbf{A}^{\prime}\right)
\end{aligned}
$$

Also using (A.69) and (A.70) we have

$$
\begin{aligned}
\operatorname{tr}\left(\mathbf{A A}^{\prime} \mathbf{B}^{2}\right) & =\frac{1}{T-1} \operatorname{tr}\left(\mathbf{A} \mathbf{A}^{\prime} \mathbf{B}\right)=\frac{1}{(T-1)^{2}} \operatorname{tr}\left(\mathbf{A A}^{\prime}\right)=\frac{\operatorname{tr}\left(\mathbf{A}^{\prime} \mathbf{A}\right)}{(T-1)^{2}}=O\left(T^{-2}\right) . \\
{\left[\operatorname{tr}\left(\mathbf{C}^{2} \mathbf{A} \mathbf{A}^{\prime}\right)\right]^{2} } & =\left[\operatorname{tr}\left(\mathbf{A} \mathbf{A}^{\prime} \mathbf{C}^{2}\right)\right]^{2} \leq \operatorname{tr}\left(\mathbf{A} \mathbf{A}^{\prime} \mathbf{A} \mathbf{A}^{\prime}\right) \operatorname{tr}\left(\mathbf{C}^{4}\right)=\operatorname{tr}\left(\mathbf{A}^{\prime} \mathbf{A} \mathbf{A}^{\prime} \mathbf{A}\right) \operatorname{tr}\left(\mathbf{C}^{4}\right) \\
& =\operatorname{tr}\left(\mathbf{C}^{2}\right) \operatorname{tr}\left(\mathbf{C}^{4}\right)=O\left(T^{-4}\right) .
\end{aligned}
$$

Finally, it is easily established that

$$
\operatorname{tr}\left(\mathbf{B}^{2} \mathbf{C}\right)=O\left(T^{-2}\right), \operatorname{tr}\left(\mathbf{C}^{3}\right)=O\left(T^{-2}\right) .
$$

Hence all the terms in (A.68) are of order $O\left(T^{-2}\right)$. 


\section{References}

Alvarez, J., Arellano, M., 2003. The time series and cross-section asymptotics of dynamic panel data estimators. Econometrica 71, 1121-1159.

Andrews, D.W.K., 1993. Exactly median-unbiased estimation of first order autoregressive/unit root models. Econometrica 61, 139-165.

Bao, Y., Ullah, A., 2006. Expectation of quadratic forms in normal and nonnormal variables with econometric applications. Unpublished manuscript, University of California, Riverside.

Hahn, J., Kuersteiner, G., 2002. Asymptotically unbiased inference for a dynamic panel model with fixed effects when both $\mathrm{n}$ and $\mathrm{T}$ are large. Econometrica 70, 1639-1657.

Hausman, J.A., 1978. Specification tests in econometrics. Econometrica 46, 1251-1271.

Holly, A., 1982. A remark on Hausman's specification test. Econometrica 50, 749-759.

Hsiao, C., 2003. Analysis of Panel Data, second edition, Cambridge University Press, Cambridge.

Hsiao, C., and Pesaran, M.H., 2007. Random coefficient panel data models. In: Matyas, L., Sevestre, P. (Eds.), The Econometrics of Panel Data, third edition. Kluwer Academic Publishers, Dordrecht, forthcoming.

Kiviet, J.F., Phillips, G.D.A., 1994. Bias assessment and reduction in linear error-correction models. Journal of Econometrics 63, 215-243.

Lieberman, O., 1994. A Laplace approximation to the moments of a ratio of quadratic forms. Biometrika 81, 681-690.

Magnus, J.R., 1978. The moments of products of quadratic forms in normal variables. Statistica Neerlandica 32, 201-210.

Magnus, J.R., 1979. The expectation of products of quadratic forms in normal variables: the practice. Statistica Neerlandica 33, 131-136.

Pesaran, M.H., 2006. Estimation and inference in large heterogeneous panels with a multifactor error structure. Econometrica 74, 967-1012.

Pesaran, M.H., 2007. A simple panel unit root test in the presence of cross section dependence. Journal of Applied Econometrics, forthcoming.

Pesaran, H., Smith, R., Im, K.S., 1996. Dynamic linear models for heterogenous panels. In: Matyas, L., Sevestre, P. (Eds.), The Econometrics of Panel Data: A Handbook of the Theory with Applications, second revised edition. Kluwer Academic Publishers, Dordrecht, pp. 145-195.

Phillips, P.C.B., Sul, D., 2003. Dynamic panel estimation and homogeneity testing under cross section dependence. Econometrics Journal 6, 217-259. 
Pitman, E.J.G., 1937. The "closest" estimates of statistical parameters. Proceedings of the Cambridge Philosophical Society 33, 212-222.

Smith, M.D., 1988. Convergent series expressions for inverse moments of quadratic forms in normal variables. The Australian Journal of Statistics 30, 235-246.

Swamy, P.A.V.B., 1970. Efficient inference in a random coefficient regression model. Econometrica $38,311-323$.

Ullah, A., 2004. Finite Sample Econometrics, Oxford University Press, Oxford.

White, H., 2001. Asymptotic Theory for Econometricians, revised edition. Academic Press, San Diego.

Zellner, A., 1962. An efficient method of estimating seemingly unrelated regressions and tests for aggregation bias. Journal of the American Statistical Association 57, 348-368. 
Table 1: Size and Power of Slope Homogeneity Tests with a Single Exogenous Regressor and Normal Errors

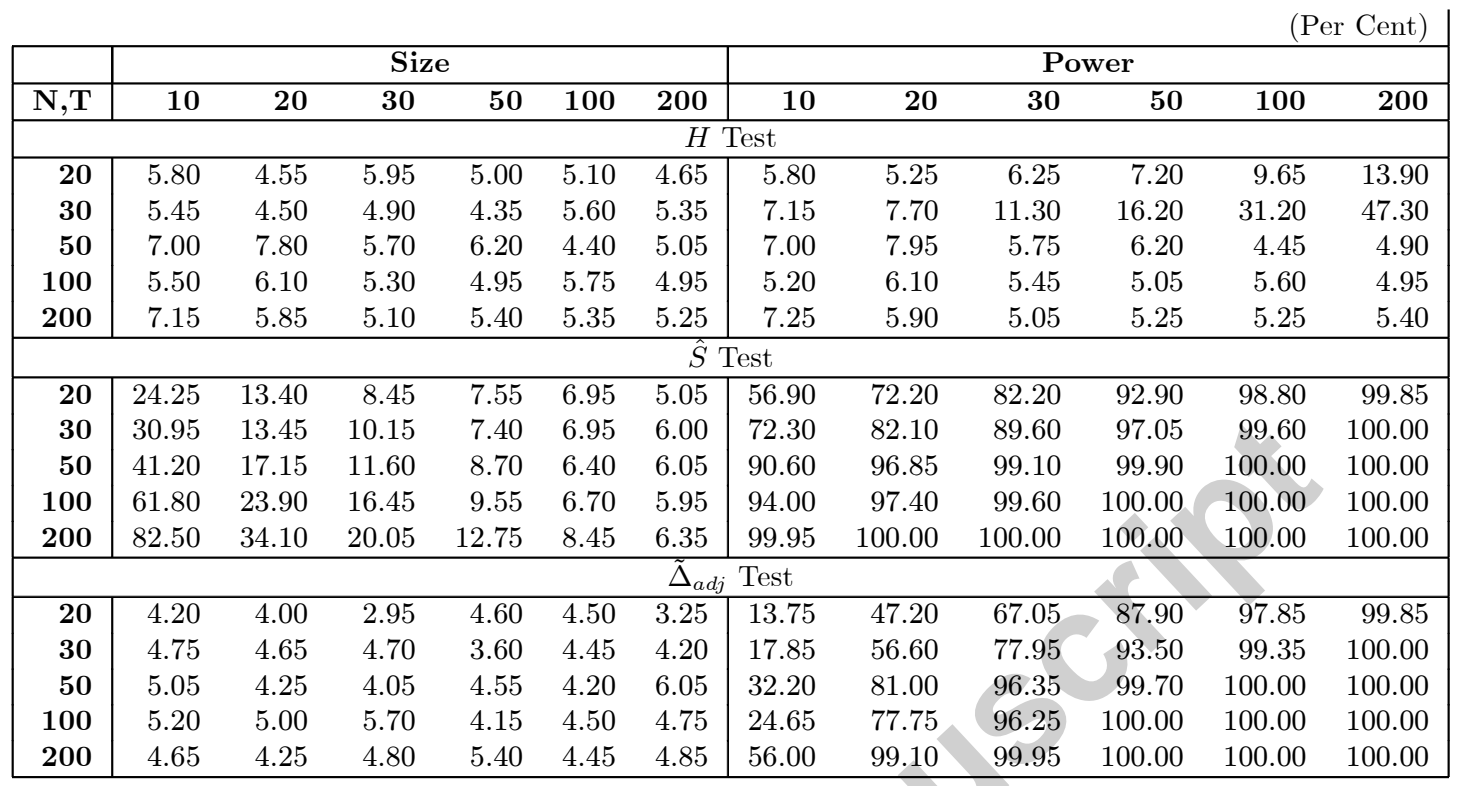

Notes: Data are generated as $y_{i t}=\alpha_{i}+x_{i t} \beta_{i}+\varepsilon_{i t}, i=1,2, \ldots, N, t=1,2, \ldots, T$, where $\alpha_{i} \sim N(1,1)$, with $x_{i t}=$ $\alpha_{i}\left(1-\rho_{i}\right)+\rho_{i} x_{i, t-1}+\left(1-\rho_{i}^{2}\right)^{1 / 2} v_{i t}, t=-48, \ldots, 0, \ldots, T, i=1,2, \ldots, N$, where $\rho_{i} \sim \operatorname{IIDU}[0.05,0.95], v_{i t} \sim \operatorname{IIDN}\left(0, \sigma_{i x}^{2}\right)$ with $\sigma_{i x}^{2} \sim I I D \chi^{2}(1) . \quad \rho_{i}$ and $\sigma_{i x}^{2}$ are fixed across replications with $x_{i,-49}=0$. The first 49 observations are discarded to reduce the effect of initial value on the generated values of $x_{i t}, t=1,2, \ldots, T$. $\varepsilon_{i t} \sim \operatorname{IIDN}\left(0, \sigma_{i}^{2}\right)$ with $\sigma_{i}^{2} \sim \operatorname{IID}\left(\chi^{2}(2) / 2\right)$. Under the null hypothesis, $\beta_{i}=1$ for all $i$, and under the alternative hypothesis $\beta_{i}=1$ for $i=1, \ldots,[N / 2]$ and $\beta_{i} \sim N(1,0.04)$ for $i=[N / 2]+1, \ldots, N$, with $[N / 2]$ being the nearest integer value of $N / 2 . \alpha_{i}$ and $\sigma_{i}^{2}$ are fixed across replications. $H$ is the heteroskedasticity robust Hausman test statistic defined by $(52) . \hat{S}$ is the Swamy's statistic defined by (11), $\tilde{\Delta}_{a d j}$ is the adjusted $\Delta$ test statistic defined by (54). $H$ and $\hat{S}$ tests are based on $\chi^{2}(k)$ and $\chi^{2}(k(N-1))$ distributions, respectively. $\tilde{\Delta}_{a d j}$ test is based on a two-sided $N(0,1)$ test. All tests are conducted at $5 \%$ nominal level, and the experiments are based on 2000 replications. 
Table 2: Size and Power of Slope Homogeneity Tests with $k=2,3$ and 4 Exogenous Regressors and Normal Errors

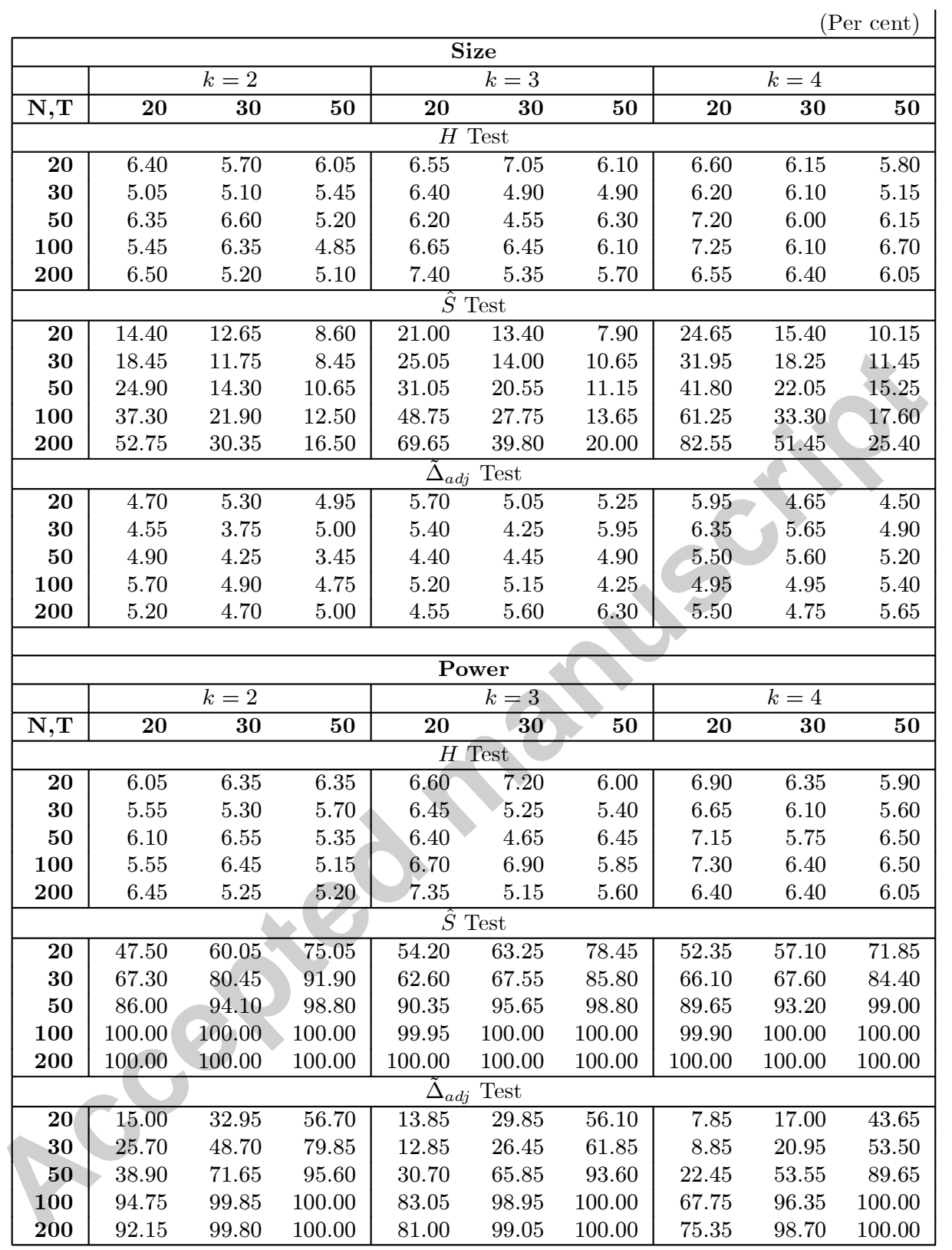

Notes: See notes to Table 1. Data are generated as $y_{i t}=\alpha_{i}+\sum_{\ell=1}^{k} x_{i \ell t} \beta_{i \ell}+\varepsilon_{i t}, i=1,2, \ldots, N, t=1,2, \ldots, T$, where $\alpha_{i} \sim N(1,1)$, with $x_{i \ell t}=\alpha_{i}\left(1-\rho_{i \ell}\right)+\rho_{i \ell} x_{i \ell, t-1}+\left(1-\rho_{i \ell}^{2}\right)^{1 / 2} v_{i \ell t}, t=-48, \ldots, 0, \ldots, T, i=1,2, \ldots, N$, where $\rho_{i \ell} \sim \operatorname{IIDU}[0.05,0.95], v_{i \ell t} \sim \operatorname{IIDN}\left(0, \sigma_{i \ell x}^{2}\right)$ with $\sigma_{i \ell x}^{2} \sim \operatorname{IID} \chi^{2}(1) . \rho_{i \ell}$ and $\sigma_{i \ell x}^{2}$ are fixed across replications with $x_{i \ell,-49}=0$. The first 49 observations are discarded. $\varepsilon_{i t} \sim \operatorname{IIDN}\left(0, \sigma_{i}^{2}\right)$ with $\sigma_{i}^{2} \sim \operatorname{IID}\left(k \chi^{2}(2) / 2\right), k=2,3,4$, so that the population $R^{2}$ of individual equations in the panel are invariant to the number of included regressors. Under the null hypothesis, $\beta_{i \ell}=1$ for all $i$ and $\ell$, and under the alternative hypothesis $\beta_{i \ell}=\beta_{i 1}$ for $\ell=2,3$, where $\beta_{i 1}=1$ for $i=1, \ldots,[N / 2]$ and $\beta_{i 1} \sim N(1,0.04)$ for $i=[N / 2]+1, \ldots, N$, with [. ] being the nearest integer value. $\alpha_{i}$ and $\sigma_{i}^{2}$ are fixed across replications. 
Table 3: Size and Power of Slope Homogeneity Tests with $k=2,3$ and 4 Exogenous Regressors and Nonnormal (Chi-Squared) Errors

\begin{tabular}{|c|c|c|c|c|c|c|c|c|c|}
\hline \multicolumn{10}{|c|}{ (Per cent) } \\
\hline \multicolumn{10}{|c|}{ Size } \\
\hline & \multicolumn{3}{|c|}{$k=2$} & \multicolumn{3}{|c|}{$k=3$} & \multicolumn{3}{|c|}{$k=4$} \\
\hline $\mathbf{N}, \mathbf{T}$ & 20 & 30 & 50 & 20 & 30 & 50 & 20 & 30 & 50 \\
\hline \multicolumn{10}{|c|}{$H$ Test } \\
\hline 20 & 6.20 & 6.00 & 5.40 & 7.05 & 6.55 & 5.70 & 7.40 & 5.55 & 5.30 \\
\hline 30 & 5.75 & 5.70 & 5.60 & 5.75 & 5.00 & 5.60 & 5.65 & 5.10 & 5.50 \\
\hline 50 & 5.30 & 6.85 & 4.90 & 7.05 & 5.60 & 5.00 & 6.65 & 5.95 & 6.00 \\
\hline 100 & 6.30 & 6.00 & 6.15 & 7.15 & 5.85 & 5.65 & 8.10 & 7.00 & 6.70 \\
\hline 200 & 6.90 & 5.60 & 6.45 & 6.35 & 5.50 & 4.10 & 7.20 & 6.45 & 5.20 \\
\hline \multicolumn{10}{|c|}{$\hat{S}$ Test } \\
\hline 20 & 15.90 & 10.40 & 8.15 & 19.50 & 12.90 & 9.45 & 24.70 & 14.10 & 9.25 \\
\hline 30 & 19.10 & 12.75 & 8.85 & 24.35 & 17.00 & 10.30 & 33.00 & 18.80 & 10.95 \\
\hline 50 & 25.75 & 14.55 & 9.65 & 31.90 & 18.45 & 12.05 & 41.15 & 24.60 & 12.95 \\
\hline 100 & 39.10 & 23.05 & 13.00 & 48.30 & 29.65 & 16.05 & 61.40 & 33.15 & 17.65 \\
\hline 200 & 54.85 & 30.45 & 17.00 & 69.50 & 40.40 & 21.75 & 81.05 & 49.50 & 23.60 \\
\hline \multicolumn{10}{|c|}{$\tilde{\Delta}_{a d j}$ Test } \\
\hline 20 & 4.15 & 3.70 & 4.25 & 4.40 & 4.35 & 5.00 & 5.90 & 5.80 & 5.60 \\
\hline 30 & 4.05 & 4.25 & 4.25 & 5.00 & 5.15 & 3.55 & 5.35 & 5.55 & 5.75 \\
\hline 50 & 4.20 & 5.00 & 4.95 & 5.35 & 4.95 & 5.60 & 5.30 & 5.60 & 5.50 \\
\hline 100 & 4.95 & 4.85 & 5.20 & 4.65 & 6.10 & 4.15 & 4.70 & 5.35 & 4.75 \\
\hline 200 & 5.50 & 4.75 & 5.30 & 4.15 & 5.65 & 5.40 & 5.60 & 5.10 & 5.45 \\
\hline \multicolumn{10}{|c|}{ Power } \\
\hline & \multicolumn{3}{|c|}{$k=2$} & \multicolumn{3}{|c|}{$k=3$} & \multicolumn{3}{|c|}{$k=4$} \\
\hline$\overline{\mathbf{N}, \mathbf{T}}$ & 20 & 30 & 50 & 20 & 30 & 50 & 20 & 30 & 50 \\
\hline \multicolumn{10}{|c|}{$H$ Test } \\
\hline 20 & 6.45 & 6.40 & 6.00 & 7.15 & 6.80 & 5.70 & 7.10 & 5.50 & 5.90 \\
\hline 30 & 5.95 & 6.15 & 6.35 & 6.60 & 5.60 & 5.95 & 6.10 & 5.45 & 5.80 \\
\hline 50 & 5.60 & 6.80 & 5.05 & 7.25 & 5.40 & 5.20 & 6.95 & 5.80 & 5.75 \\
\hline 100 & 6.10 & 5.95 & 6.10 & 7.15 & 5.80 & 6.20 & 8.05 & 6.85 & 6.65 \\
\hline 200 & 6.95 & 5.60 & 6.55 & 6.45 & 5.55 & 4.25 & 7.20 & 6.60 & 5.35 \\
\hline \multicolumn{10}{|c|}{$\hat{S}$ Test } \\
\hline 20 & 55.75 & 62.95 & 76.40 & 58.10 & 67.45 & 79.85 & 57.05 & 60.10 & 73.05 \\
\hline 30 & 75.75 & 81.70 & 93.70 & 66.70 & 73.80 & 87.35 & 70.40 & 73.65 & 86.10 \\
\hline 50 & 90.55 & 94.40 & 98.60 & 90.70 & 95.20 & 98.95 & 92.95 & 95.80 & 98.65 \\
\hline 100 & 99.95 & 100.00 & 100.00 & 99.95 & 100.00 & 100.00 & 100.00 & 100.00 & 100.00 \\
\hline 200 & 100.00 & 100.00 & 100.00 & 100.00 & 100.00 & 100.00 & 100.00 & 100.00 & 100.00 \\
\hline \multicolumn{10}{|c|}{$\tilde{\Delta}_{a d j}$ Test } \\
\hline 20 & 19.85 & 33.95 & 59.75 & 15.50 & 30.45 & 60.40 & 9.10 & 20.00 & 46.60 \\
\hline 30 & 29.95 & 54.30 & 82.10 & 16.05 & 33.75 & 67.20 & 12.95 & 22.85 & 56.35 \\
\hline 50 & 47.90 & 75.75 & 95.20 & 35.70 & 70.80 & 94.20 & 26.85 & 61.20 & 91.55 \\
\hline 100 & 97.05 & 100.00 & 100.00 & 89.50 & 98.90 & 99.95 & 76.30 & 97.50 & 100.00 \\
\hline 200 & 96.60 & 100.00 & 100.00 & 87.60 & 99.35 & 100.00 & 82.65 & 99.40 & 100.00 \\
\hline
\end{tabular}

Notes: See notes to Table 1. Data are generated in the same way as specified in Table 2, except $\varepsilon_{i t}=\sigma_{i} u_{i t}$ with $u_{i t} \sim I I D\left(\left(\chi^{2}(2)-2\right) / 2\right), \sigma_{i}^{2} \sim I I D\left(k \chi^{2}(2) / 2\right)$. 


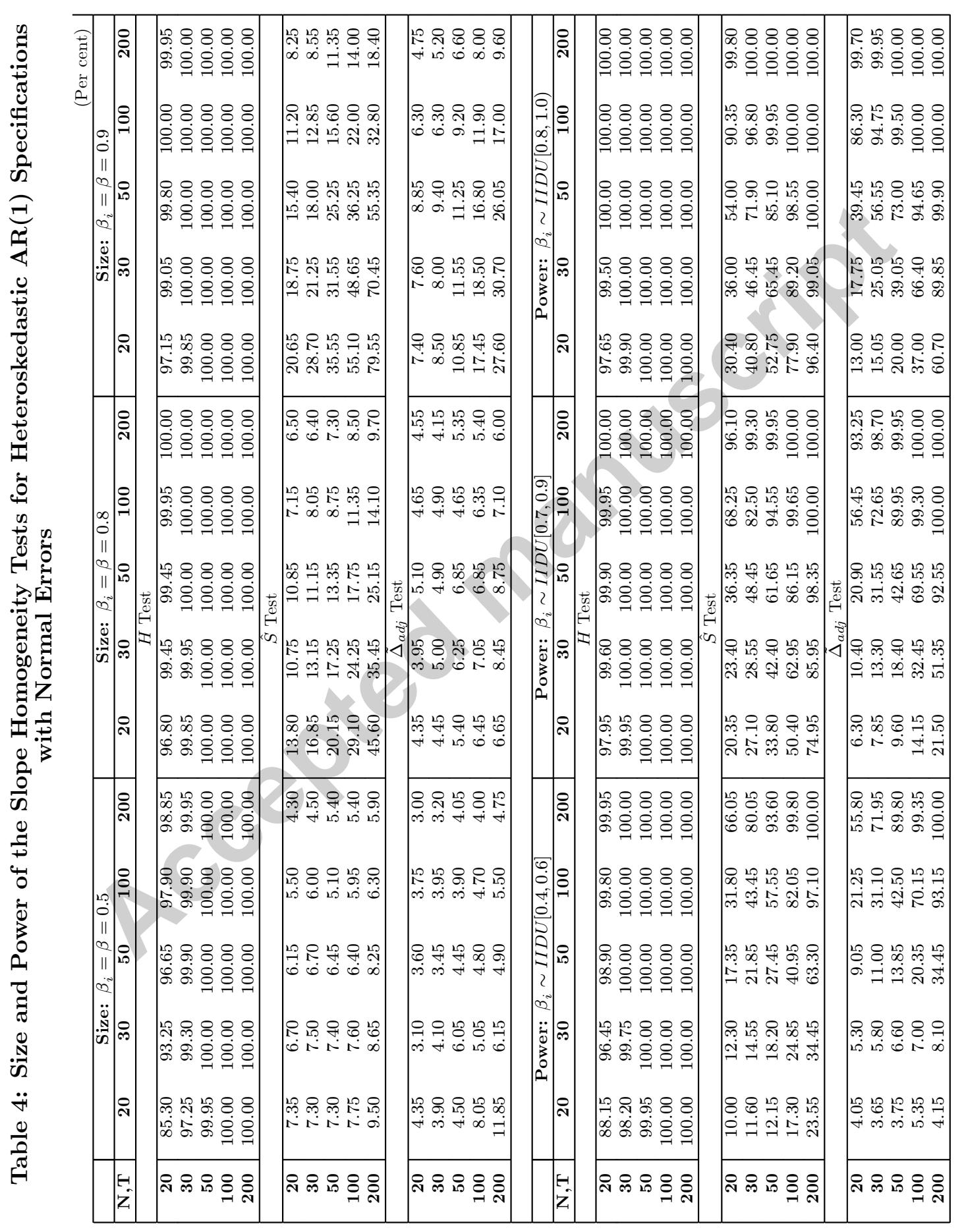




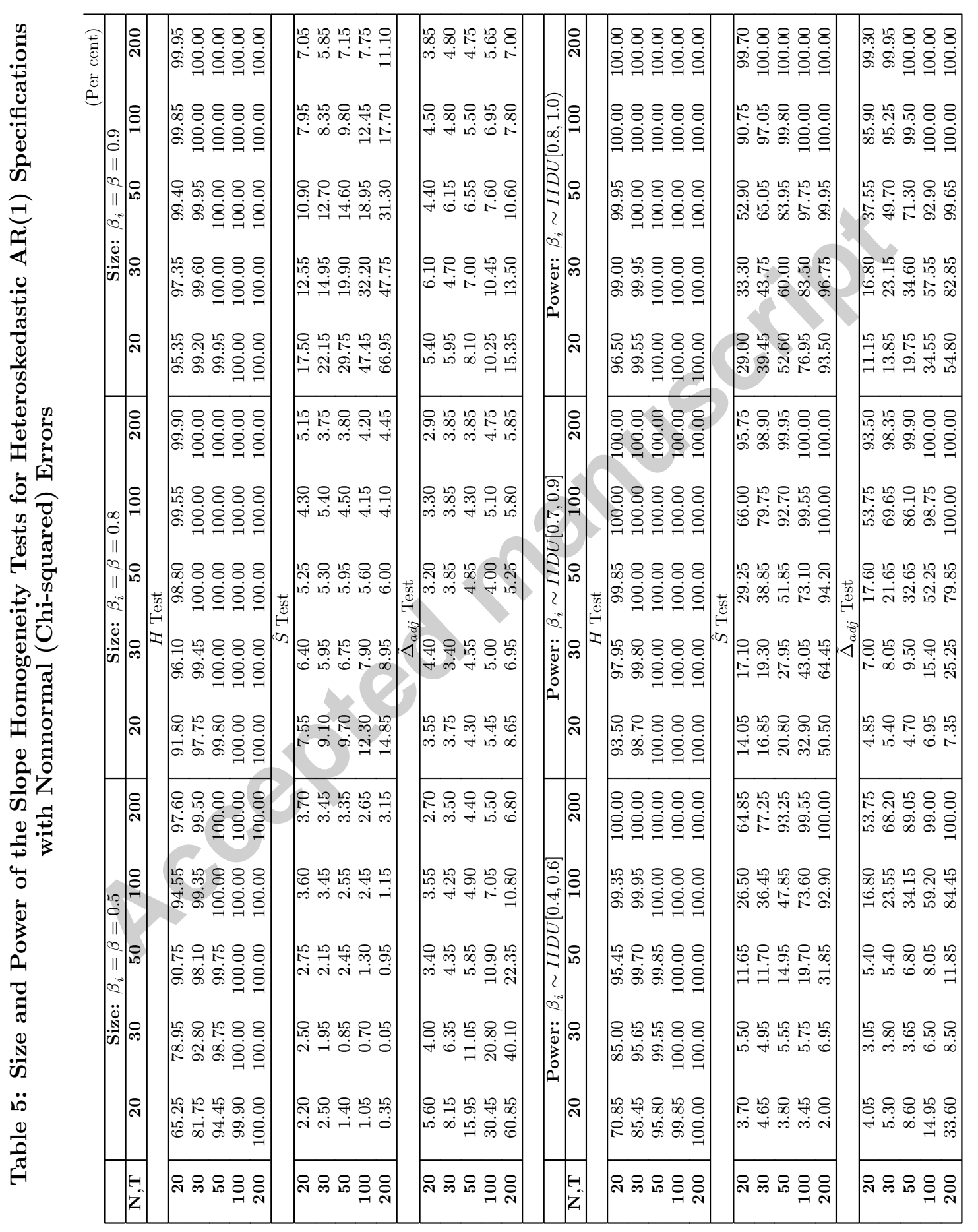




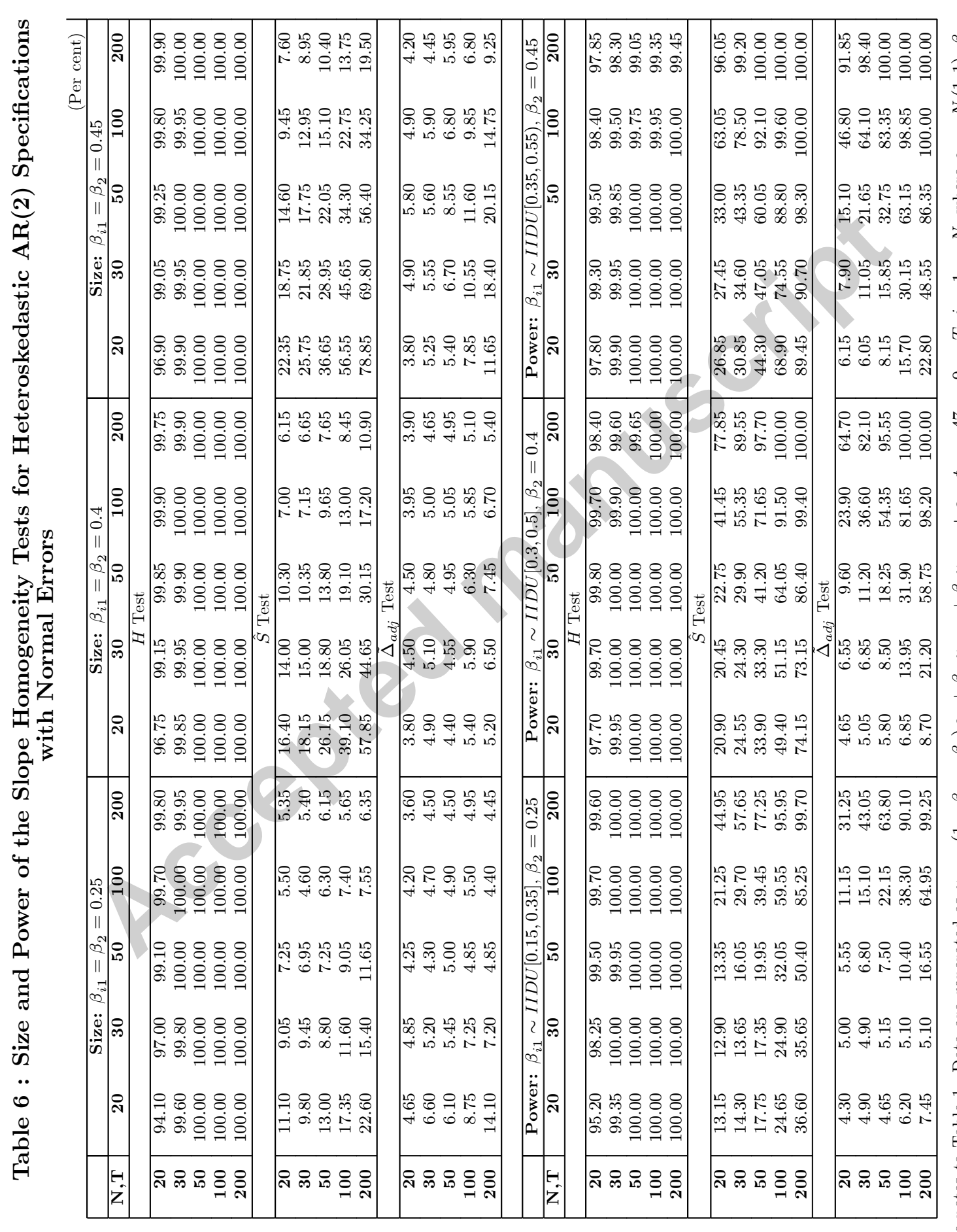

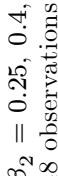

$\mathrm{\aleph}^{2} \infty$

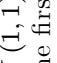

乙.

8ं

事离

$\vdots \stackrel{+}{1}$

$\sim \frac{\pi}{3}$

Ais

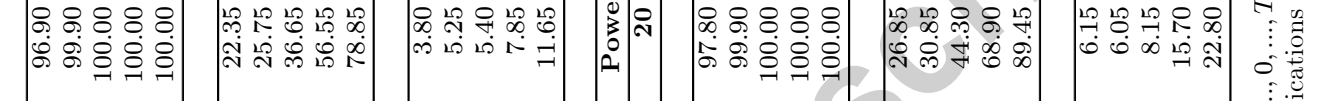

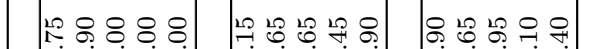

일

$8 \quad \therefore 88.8$

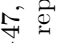

$\stackrel{-1}{0}$

$\exists=$

$+\stackrel{4}{c}$

I

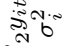

逗 


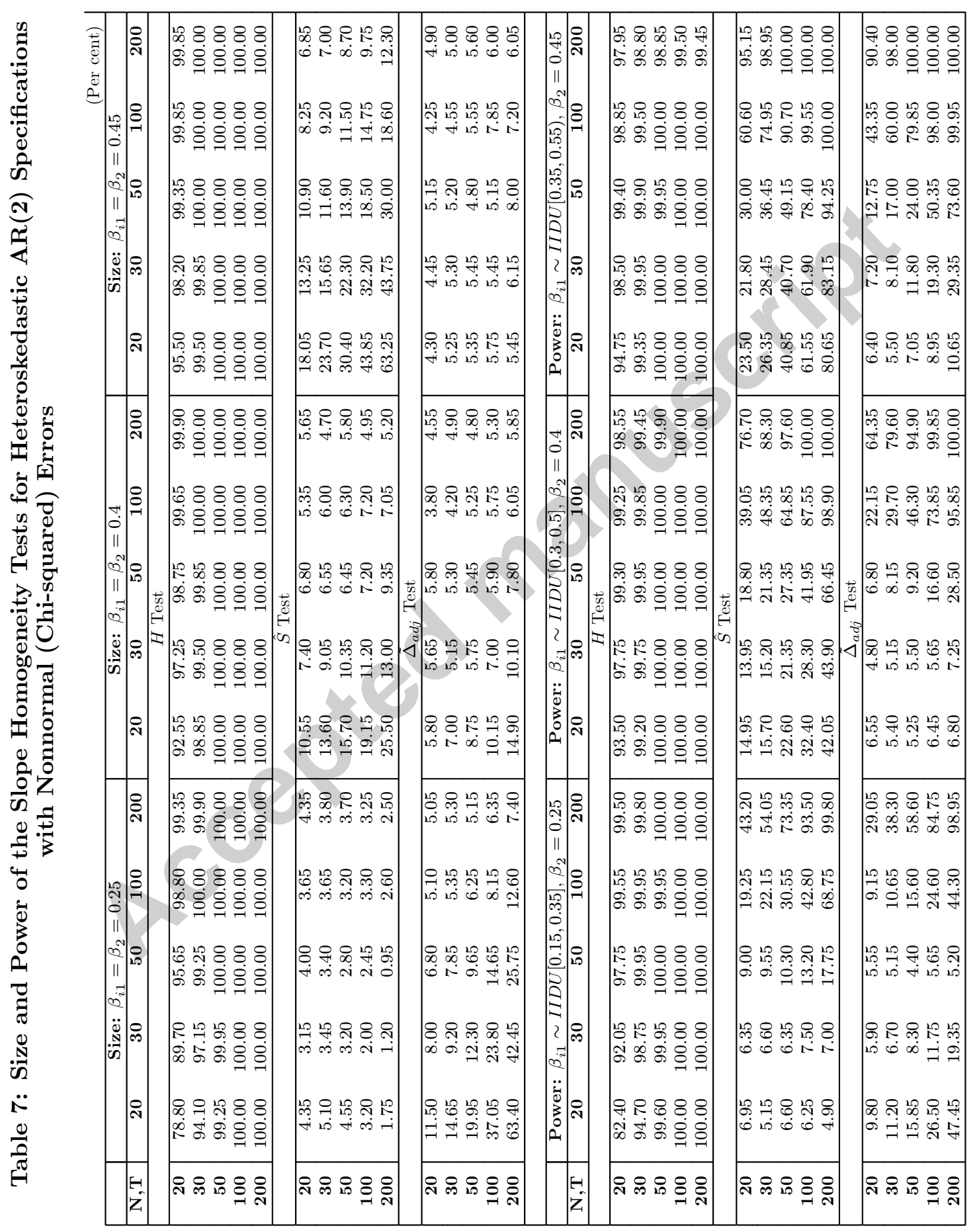



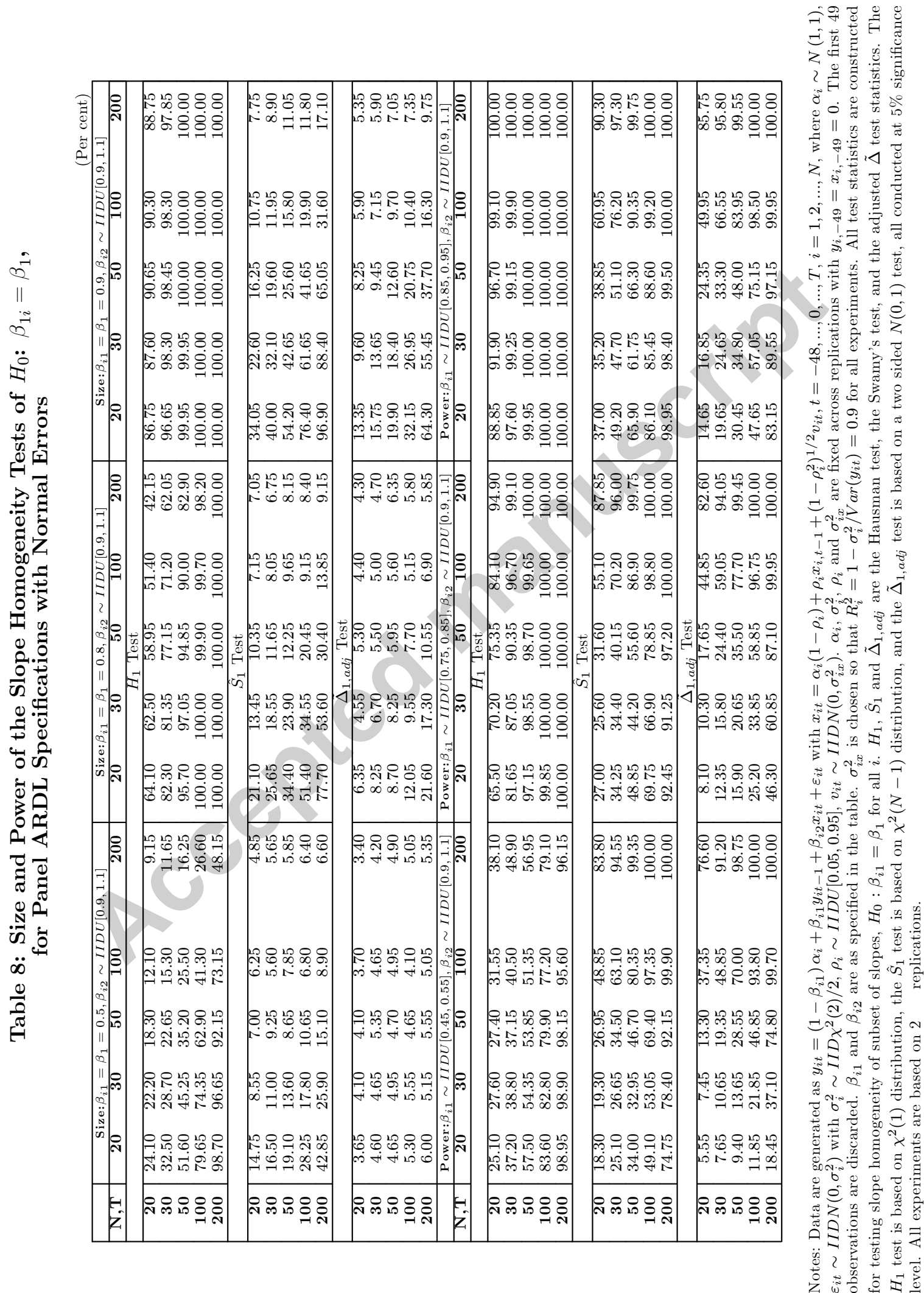


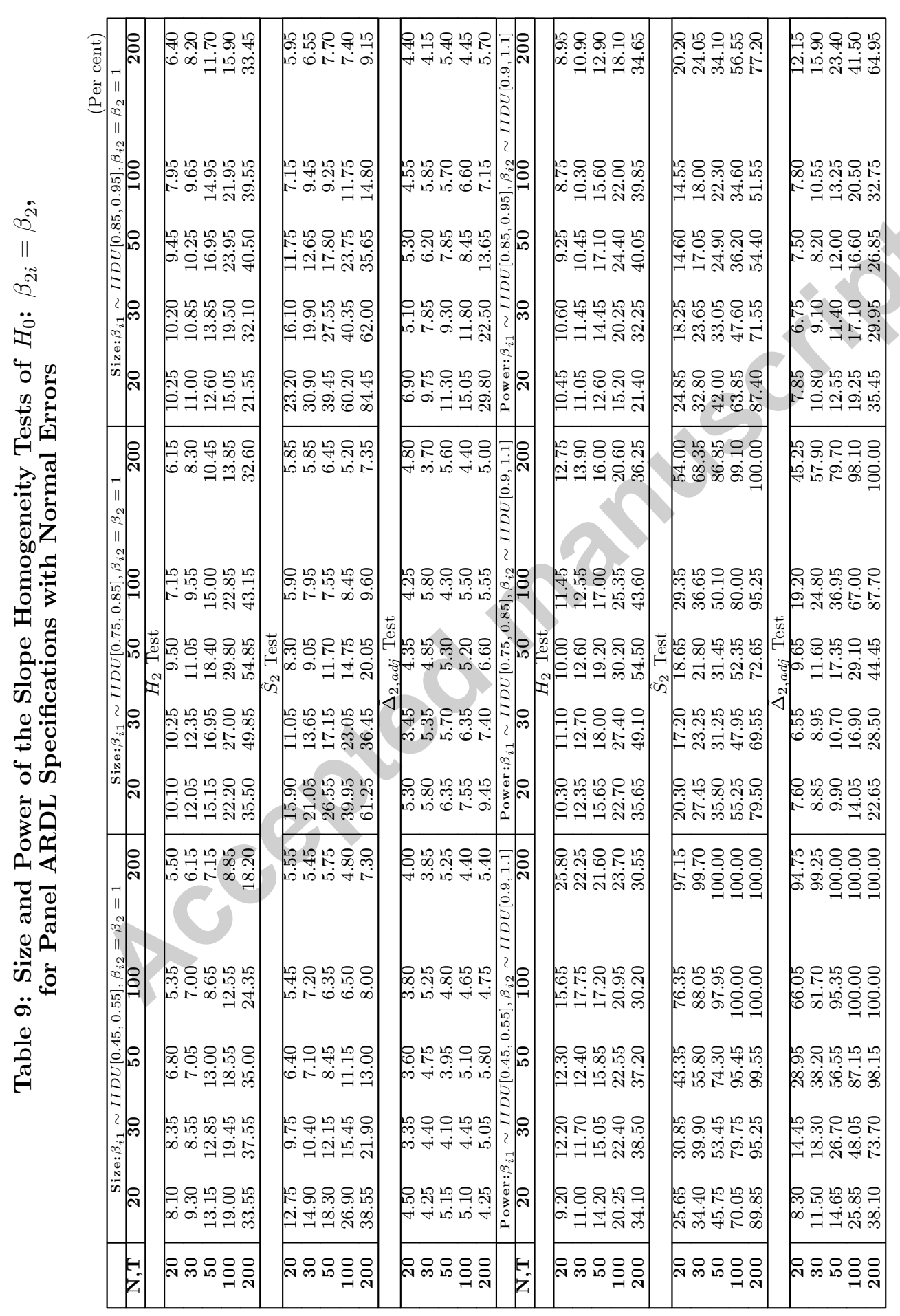

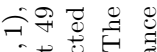

E范

\&

붕

80 要

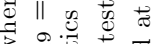

ᄀ.

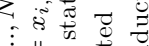

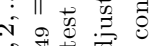

i $=$

$\therefore$ तय

ㅌ一⿱

o :

중

† 항

Iㅐ

더웡

i

नis

인

$=$.

$+2 \cdot 0.0$

1. 1 \&

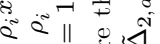

+ $\begin{gathered}5 \\ 0\end{gathered}$

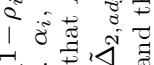

$8 \mathrm{\sigma}: \square$

包

द्य

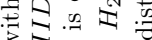

$=2 \pi .2$.

के

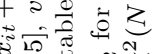

20.

i० 0

$+0 . \exists$ 잉

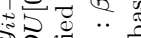

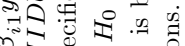

0
+2
+

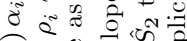

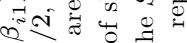

I बิ

$\|$ 政者

ज记

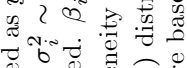

政

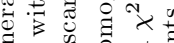

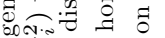

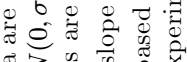

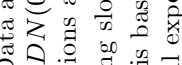

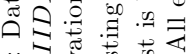

论人

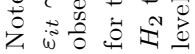




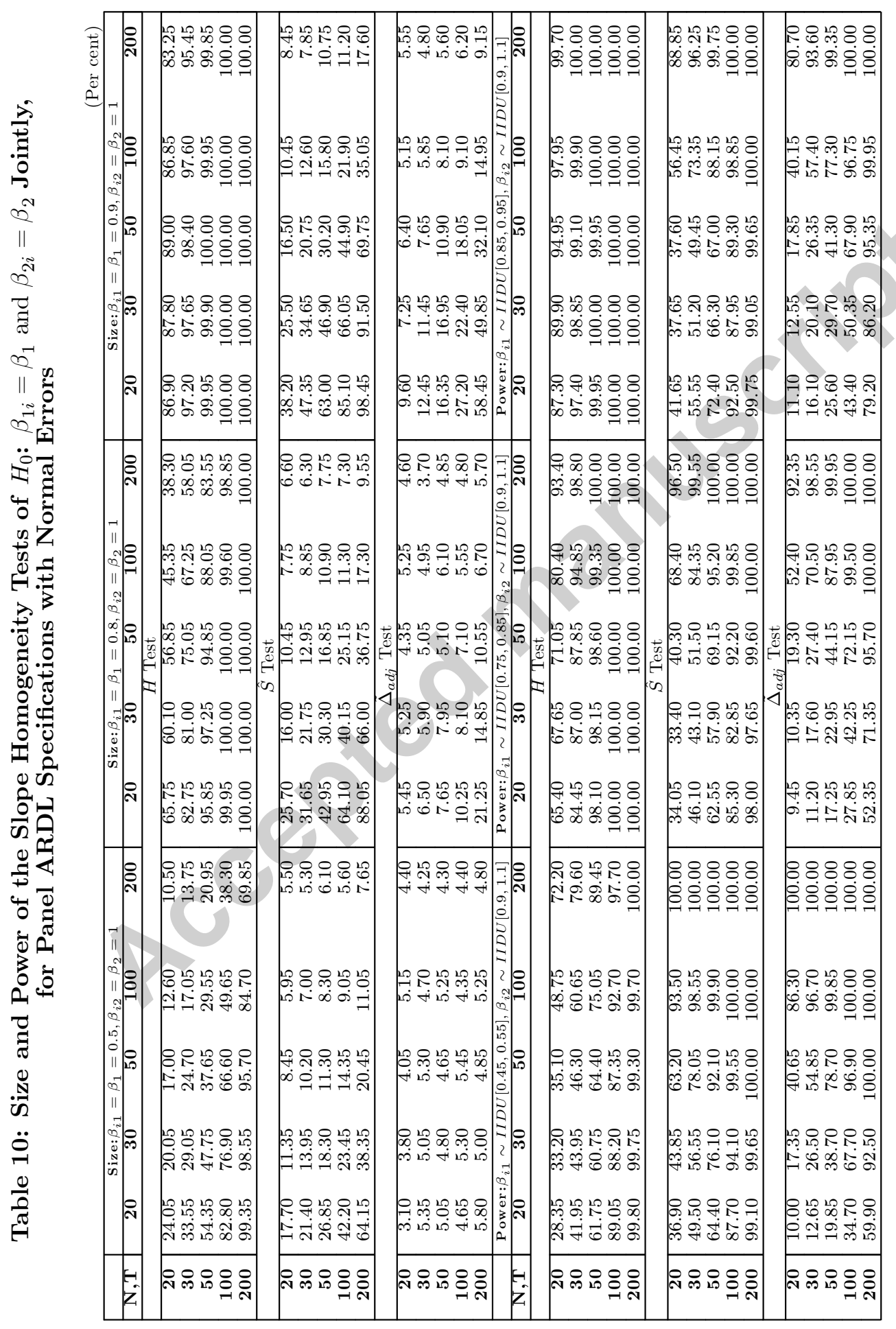

하요 궁

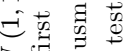
2 每 र 000 范 $z y^{-1}$ ఏ $\|$ ก 9 i $\|$ 政

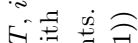
$\begin{array}{ccc}\vdots & 0 & 0 \\ \vdots & 0 & 0 \\ 0 & 0 & 0\end{array}$ $\vdots$ ำ $\infty: 0$. 1 矛范 $\|$ 零

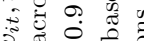
N 0 || तुल 1 हैं

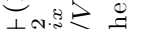
I.

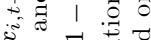

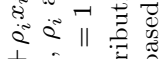
+ a d ชิ

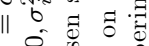
eी 플. 근. $=2$ 중

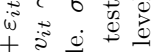
政霆 तูก 0 लं0 $+20 . \approx 00.00$

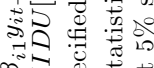

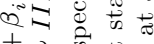
+2 की

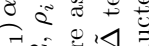
लेखें । ลิ 웡 चN

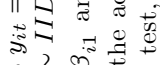
वै

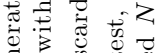

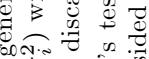

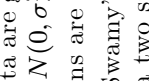

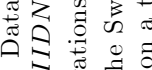

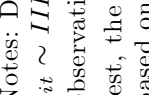

